\title{
Polymer Combustion as a Basis for Hybrid Propulsion: A Comprehensive Review and New Numerical Approaches
}

\author{
Vasily Novozhilov ${ }^{1}{ }^{*}$, Paul Joseph ${ }^{1}$, Keiichi Ishiko ${ }^{2}$, Toru Shimada ${ }^{3}$, Hui Wang ${ }^{4}$ and Jun Liu ${ }^{4}$ \\ 1 Fire Safety Engineering Research and Technology Centre, School of the Built Environment, \\ Built Environment Research Institute, University of Ulster, Newtownabbey, BT37 0QB, UK; \\ E-Mail: p.joseph@ulster.ac.uk \\ 2 Aerospace Research and Development Directorate, Japan Aerospace Exploration Agency, \\ 7-44-1 Jindaiji-Higashi, Chofu, Tokyo 182-8522, Japan; E-Mail: ishiko.keiichi@jaxa.jp \\ 3 Institute of Space and Astronautical Science, Japan Aerospace Exploration Agency, \\ 3-1-1 Yoshinodai, Chuo, Sagamihara, Kanagawa 252-5210, Japan; E-Mail: shimada.toru@jaxa.jp \\ 4 School of Computing and Mathematics, Computer Science Research Institute, University of Ulster, \\ Newtownabbey, BT37 0QB, UK; E-Mails: h.wang@ulster.ac.uk (H.W.); j.liu@ulster.ac.uk (J.L.) \\ * Author to whom correspondence should be addressed; E-Mail: VB.Novozhilov@ulster.ac.uk; \\ Tel.: +44-28-9036-8705; Fax: +44-28-9036-8726.
}

Received: 28 July 2011; in revised form: 11 October 2011 / Accepted: 12 October 2011 / Published: 24 October 2011

\begin{abstract}
Hybrid Propulsion is an attractive alternative to conventional liquid and solid rocket motors. This is an active area of research and technological developments. Potential wide application of Hybrid Engines opens the possibility for safer and more flexible space vehicle launching and manoeuvring. The present paper discusses fundamental combustion issues related to further development of Hybrid Rockets. The emphasis is made on the two aspects: (1) properties of potential polymeric fuels, and their modification, and (2) implementation of comprehensive CFD models for combustion in Hybrid Engines. Fundamentals of polymeric fuel combustion are discussed. Further, steps necessary to accurately describe their burning behaviour by means of CFD models are investigated. Final part of the paper presents results of preliminary CFD simulations of fuel burning process in Hybrid Engine using a simplified set-up.
\end{abstract}

Keywords: hybrid propulsion; polymeric fuels; combustion; computational fluid dynamics 


\section{Nomenclature}

$\vec{A} \quad$ flux Jacobian matrix, $=\partial \vec{E} / \partial \vec{Q}$

$A_{O} \quad$ mass fraction of element $O$ in the air $\left(0 \leq A_{O} \leq 1\right)$

$b_{i}^{0} \quad$ mole number for $i$-th element per unit mass of mixture gas [kgmol/kg]

$c_{p}, c_{p}(T) \quad$ specific heat at constant pressure $[\mathrm{J} /(\mathrm{kg} \mathrm{K})]$

$c_{p j}(T) \quad$ specific heat at constant pressure for $j$-th chemical species $[\mathrm{J} /(\mathrm{kg} \mathrm{K})]$

$c_{v}(T) \quad$ specific heat at constant volume $[\mathrm{J} /(\mathrm{kg} \mathrm{K})]$

$C_{D} \quad$ drag coefficient

$c_{f} \quad$ friction coefficients

$C_{\mu} \quad k-\varepsilon$ model constant

$d \quad$ diameter [m]

$D \quad$ diffusion coefficient $\left[\mathrm{m}^{2} / \mathrm{s}\right]$

$D_{j} \quad$ subgrid scale (SGS) viscous diffusion

$e \quad$ total energy per unit volume $\left[\mathrm{J} / \mathrm{m}^{3}\right]$

$e_{\text {in }} \quad$ internal energy $[\mathrm{J} / \mathrm{kg}]$

$E \quad$ total energy per unit mass $[\mathrm{J} / \mathrm{kg}]$

$\vec{E} \quad$ inviscid flux vector

$\vec{E}_{i+1 / 2} \quad$ numerical flux vector at the cell interface

$\vec{E}^{ \pm} \quad$ obtained flux by flux vector splitting methods

$G \quad$ kernel of filter

$h \quad$ enthalpy per unit mass $[\mathrm{J} / \mathrm{kg}]$

$h_{C} \quad$ convective heat transfer coefficient $\left[\mathrm{W} / \mathrm{m}^{2} \mathrm{~K}\right]$

$h_{j}(T) \quad$ internal enthalpy per unit mass $j$-th chemical species $[\mathrm{J} / \mathrm{kg}]$

$H \quad$ total enthalpy per unit mass $[\mathrm{J} / \mathrm{kg}]$

I total radiation intensity $\left[\mathrm{W} / \mathrm{m}^{2} / \mathrm{sr}\right]$

$J_{j} \quad$ subgrid scale (SGS) turbulent diffusion

$k$ parameter deciding the spatial accuracy of MUSCL;

turbulent kinetic energy $\left[\mathrm{m}^{2} / \mathrm{s}^{2}\right]$

$L_{f g} \quad$ latent heat of liquid evaporation $[\mathrm{kJ} / \mathrm{kg}]$

$m_{i} \quad$ mass of $i$-th element $[\mathrm{kg}]$

$m_{j} \quad$ mass of $j$-th molecule $[\mathrm{kg}]$

$\dot{m} \quad \operatorname{mass}$ flux $\left[\mathrm{kg} /\left(\mathrm{m}^{2} \mathrm{~s}\right)\right]$

$\dot{m}_{p} \quad$ pyrolysis rate $\left[\mathrm{kg} /\left(\mathrm{m}^{2} \mathrm{~s}\right)\right]$

$\mathrm{Nu} \quad$ Nusselt number

$n \quad$ mole number for mixture gas per unit mass of mixture gas [kgmol/ $\mathrm{kg}]$

$\vec{n} \quad$ inner normal to fuel surface

$n_{j} \quad$ mole number for $j$-th species per unit mass of mixture gas [kgmol/kg]

$n_{x}, n_{y}, n_{z} \quad$ Cartesian components of a normal vector from the left to the right at cell interface

$n_{j} \quad$ component of normal vector to $\partial \Omega$ 


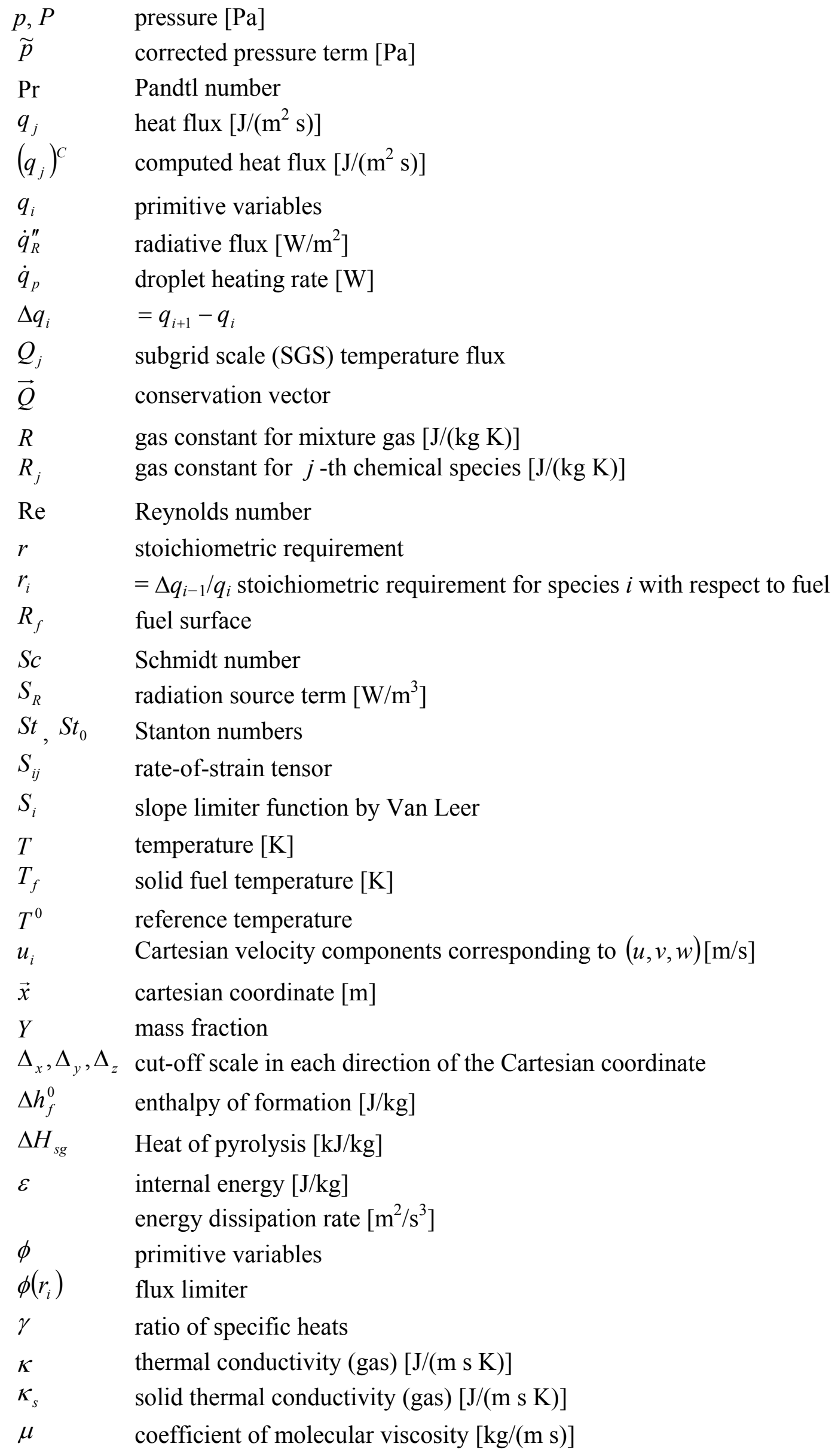




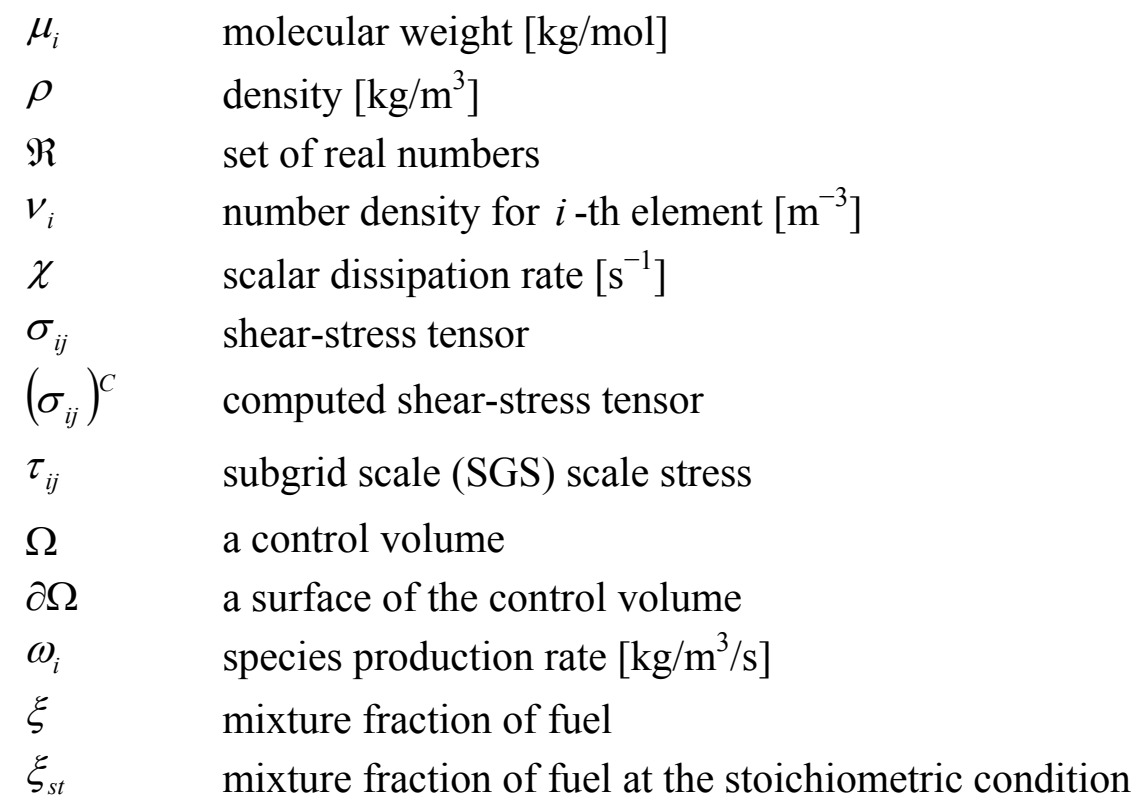

\section{Constant values}

$\begin{array}{ll}N_{A} & \text { Avogadro constant }=6.02214179\left[\mathrm{~mol}^{-1}\right] \\ g & \text { gravity acceleration }=9.81\left[\mathrm{~m}^{2} / \mathrm{s}\right] \\ R^{0} & \text { the universal gas constant }=8314.51[\mathrm{~J} /(\mathrm{kgmol} \mathrm{K})] \\ \sigma & \text { Stefan-Boltzmann constant }=5.67010^{-8}\left[\mathrm{~W} / \mathrm{m}^{2} / \mathrm{K}^{4}\right]\end{array}$

\section{Superscripts}

$\begin{array}{ll}\mathrm{F} & \text { fuel } \\ \mathrm{g} & \text { gas } \\ 1 & \text { liquid } \\ n & \text { the time step } \\ \mathrm{O} & \text { oxygen } \\ \mathrm{pr} & \text { product } \\ \mathrm{S} & \text { surface } \\ \mathrm{SGS} & \text { sub-grid scale } \\ \rightarrow & \text { vector } \\ - & \text { filtered value } \\ ()^{F} & \text { Favre averaged value } \\ ()^{C} & \text { value computed by the Favre averaged values } \\ \infty & \text { Free stream } \\ \cap & \text { point of curves intersection } \\ \| & \text { norm } \\ (\cdot) & \text { dot product }\end{array}$

\section{Subscripts}

$i, j \quad$ direction in the Cartesian coordinate system $(i, j=1,2,3)$

$i \quad$ elements $\mathrm{C}, \mathrm{H}, \mathrm{O}$ and $\mathrm{N}$

$j \quad$ chemical species $\mathrm{C}_{x} \mathrm{H}_{y}, \mathrm{O}_{2}, \mathrm{CO}_{2}, \mathrm{H}_{2} \mathrm{O}$ and $\mathrm{N}_{2}$ 


\section{Introduction}

A revived interest in hybrid propulsion systems has become apparent in recent years [1]. It is well known that traditional liquid and solid rocket motors suffer from certain drawbacks. For example, liquid systems, while providing high performance, require quite complex and costly plumbing. Solid systems, on the other hand, are inherently unsafe due to uniform mixing of fuel and oxidizer. Besides, there are difficulties with thrust control and termination in such engines.

Hybrid systems aim to provide alternative to both abovementioned technologies by executing combustion in diffusion mode, and therefore with initial separation of fuel and oxidizer. In a classical design, solid fuel and liquid oxidizer are used. Such arrangement provides operationally flexible, safe and reasonably priced solution. A variety of solid fuels, such as hydrocarbons and metals, and a wide range of liquid oxidizers can be used.

Up to now, various modifications of the basic idea have been proposed, such as reverse hybrid, tribrid, solid-fuel ramjet, and the ducted rocket. Reverse hybrids use solid oxidizer and liquid fuel. The solid oxidizer has to be mixed with either an inert filler or a small percentage of fuel. The tribrid systems use bipropellant combustion with a third component being metalized fuel. The solid-fuel ramjet utilizes a solid fuel and rams air as an oxidizer. Finally, the ducted rocket uses fuel rich solid propellant to generate a flame, which mixes downstream with the ram air to achieve optimum performance.

Experience with Hybrid Rockets dates back to 1930s, with the first attempt reported in Russia. The earliest demonstrations of flight worthy hybrid rockets are due to Pacific Rocket Society from mid1940s to early 1950s. During the 1960s, hybrid sounding rocket tests were conducted in France and Sweden. Since then, significant progress has been observed [2]. Latest significant developments were the Hyperion Sounding Rocket (1996-97) and Lockheed Martin Corporation (2002) programs.

The classical hybrid rocket propulsion posses a number of very attractive features. Its advantages over solid and liquid propulsion technologies can be summarised as follows:

(1) Safety. This is a major attraction. The solid fuel is inert, therefore it can be manufactured, transported and handled safely. In addition, because an intimate mixture of oxidizer and fuel is not possible, it is non-explosive.

(2) Operating issues. Engine throttling and shutdown are significantly simplified by this technology. Throttling is achieved by liquid flow rate modulation, which is considerably simpler in this case compared to a liquid rocket engine where two liquid streams have to be synchronised. Termination is accomplished by cutting of the liquid flow rate. This opens possibility of quick and robust abort procedure.

(3) Choice of fuel. A wide range of easily available solid fuels can be used, giving wider design flexibility compared to liquid or solid motors. Combustion performance of solid fuel is also more reliable since in a hybrid mode it is not sensitive to fuel-grain cracks.

(4) Cost. Operational costs are obviously of great importance. In this regard hybrid systems benefit from simplified manufacturing procedures, due to their inherent safety. Consequently, fabrication (and therefore operation) costs are reduced. 
As for the disadvantages, the major of them are:

(1) Low regression rate. This is a major obstacle for a wide use of Hybrid Rocket Engines. Essentially non-energetic nature of fuels gives rise to requirement of very high regression rates, in order to achieve required thrust. In practice it leads to necessity to use multiple ports, and other modifications that complicates design.

Related consequence is a low fuel bulk density (i.e., volumetric fuel loading).

(2) Combustion efficiency is lower compared to liquid or solid engines, due to non-premixed nature of combustion.

(3) Finally, ignition transient and thrust response to throttling is slower than in solid or liquid motors.

Despite the above shortcomings, hybrid propulsion technology enjoys quite versatile application potential. They include, but are not limited to, for example, sounding rockets, auxiliary power units, tactical rockets, space engines, thrust augmentation, and large launch boosters.

In particular, research on Hybrid Propulsion Engines is concentrated within several research clusters around the World. For example, research in Europe has been supported by the European Commission via ORPHEE project (http://www.orphee-fp7-space.eu/index.html) under FP7 Research Program. More specific applied details may be obtained directly from the participants of that project.

As indicated, enhancement of regression rates is a primary obstacle for wide spread of technology. Consequently, significant research efforts are currently concentrated in this area.

Further progress requires, first of all, advanced understanding of combustion properties of various polymeric fuels, and potential ways of their modification. Secondly, regression rates and overall Hybrid Engine performance are determined by the global dynamics of combustion process in the engine. This process also has to be understood quite well. Computational Fluid Dynamics (CFD) modelling becomes increasingly important tool for such understanding. The challenge of predicting combustion process in Hybrid Engine (Figure 1) from the first principles is quite challenging.

Figure 1. Schematic of the combustion process in a Hybrid Rocket Engine.

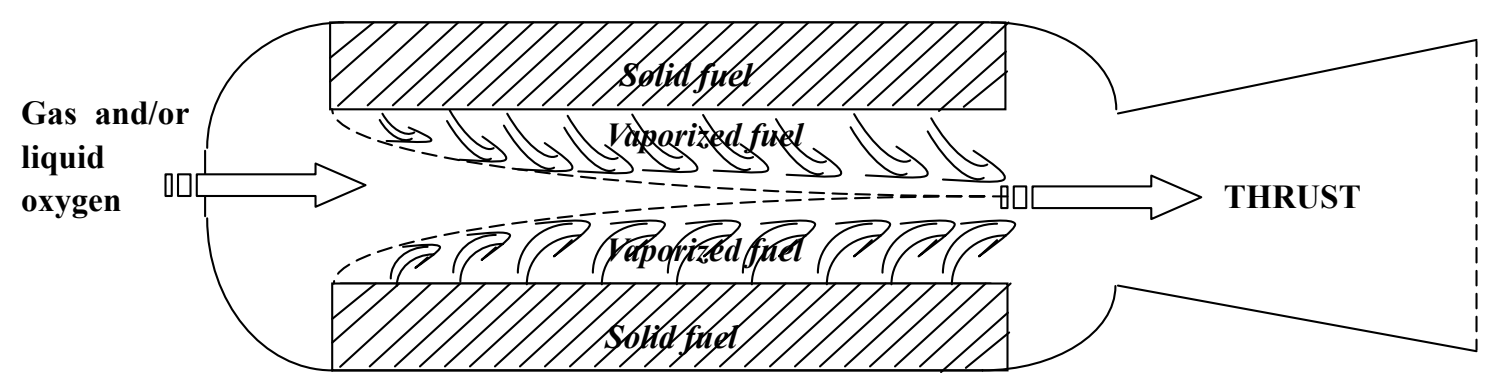

According to the above considerations, the purpose of this paper is to discuss properties of polymeric fuels, relevant to their implementation in hybrid propulsion, and also indicate the ways towards development of comprehensive CFD models for Hybrid Rocket Engines. The paper is arranged in the following manner: fundamentals of polymer combustion are discussed in Section 2. Here, among other issues, potential ways to chemical modification of existing fuels are discussed. This 
is followed by Section 3, where a roadmap towards comprehensive CFD modelling of combustion process in hybrid systems is presented. This section mostly focuses on implementation of relevant combustion models for solid fuel degradation and turbulent diffusion flames. Several other necessary models are also discussed here. Section 4 contains results of preliminary CFD modelling, developed along the lines presented in Section 3.

\section{Fundamentals of Polymer Combustion}

\subsection{Flammability Characteristics of Polymeric Materials}

When subjected for a sufficient length of time to an external heat source, organic polymeric materials undergo thermal degradation, generating various products in varying concentrations over different temperature ranges. The nature and amount of the volatile combustible products depend on the chemical structures of the polymers undergoing degradation. If the gaseous mixture resulting from the mixing of degradation volatiles with air is within the flammability limits, and the temperature is above the ignition temperature, then combustion begins (Figure 2). The combustion of a polymeric material is a highly complex process involving a series of interrelated and/or independent stages occurring in the condensed phase and the gaseous phase, and at the interfaces between the two phases [3].

Figure 2. Combustion cycle of a polymeric material.

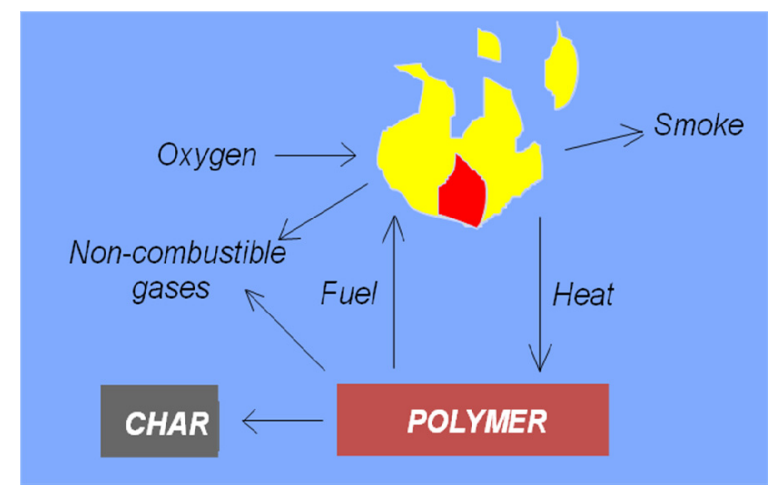

The chemical steps leading to the formation of volatiles may be homolytic or heterolytic, i.e., be radical or ionic [4]. The three overall processes implicated in the thermal degradation of most thermoplastic polymers are as follows:

1. Random chain cleavage followed by chain unzipping is characterized by high monomer yields and a slow decrease in the molecular weight of the polymer, e.g., poly(methyl methacrylate), poly $(\alpha$-methylstyrene), polystyrene,polytetrafluoroethylene.

2. Random chain cleavage followed by further chain scission is characterized by very low monomer yields amongst the degradation products and a rapid drop in molecular weight, e.g., polyethylene, polypropylene, poly(methylacrylate), polychlorotrifluoroethylene.

3. An intra-chain chemical reaction followed by cross-linking reaction and formation of a carbonaceous residue, or random chain cleavage. This generates a relatively high yield of volatiles from the intra-chain reaction, but produces little monomer, and produces, no, or only 
a very slight, reduction in molecular weight during the initial stages of degradation, e.g., poly(vinyl chloride), poly(vinyl alcohol), polyacrylonitrile.

In some cases, several of these processes occur simultaneously, depending on the sample size, heating rate, pyrolysis temperature, environment, and presence of any additives. Although polymer degradation schemes can be greatly altered by the presence of comonomers, side-chain substituents, and other chemical constituent factors, the ultimate thermal stability is determined by the relative strengths of the main-chain bonds. Many additives and comonomers employed as flame retardants are thermally liable; as a result the thermal stability of the polymer system is reduced. In order to reduce the observed effects of the flame retardant additives on the thermal stability of the polymeric materials, more thermally stable and hence inherently fire-resistant polymers are of increasing interest.

Thermosets, on the other hand, owing to the highly cross-linked three dimensional structural attribute, have much lesser propensity to thermally degrade (especially at lower temperatures) to yield flammable volatiles. The rupture of bonds does not initially generate combustible gases, and carbonisation is usually promoted. Ladder polymers, in which the main chains are bonded together at each repeat unit by a cross-link, serve the same purpose. Polymers with relatively strong main chain bonds and/or with aromatic and heterocylic structural units, are also inherently thermally stable [5]. There are several classes of polymers, such as polyphenylenes, poly( $p$-phenylene oxide)s, polybenzimidazoles, polybenzamides, that have relatively high thermal decomposition temperatures coupled to low levels of fuel production on degradation.

The ability to form char is related to the flammability of a polymer. The higher the amount of residual chars after combustion, the lower the amount of combustible material available to perpetuate the flame and greater is the degree of flame retardance of the material [6]. Therefore, one of the ways to achieve high degrees of flame retardancy or non-combustibility of polymeric materials is to increase the amount of char produced on combustion. This is illustrated by the fact that aromatic polymers, e.g., polycarbonate and poly(phenylene oxide), have lower flammabilities than purely aromatic polymers. The greater thermal stability of cross-linked and aromatic structures in thermosets gives rise to greater degree of condensation into aromatic chars, and therefore only relatively low levels of flammable gases are available to feed the flame.

As a consequence of the complex nature and pure reproducibility of fire, there are many techniques for estimating the flammability characteristics of polymeric materials. The most widely used laboratory test is the Limiting Oxygen Index (LOI) measurement, which is a very convenient, precise and reproducible technique [7]. The LOI is a measure of the volume percentage of oxygen in a mixture of oxygen and nitrogen gas stream that just supports candle-like combustion of a polymer mixture. This value therefore enables the combustibility of a polymer to be expressed and compared with that of other material (Table 1). However, the relatively high concentrations of oxygen used in making measurements of LOI are unrepresentative of a real fire, and generally there is a lack of correlation between most of the small-scale test and full-scale tests. 
Table 1. Limiting oxygen indices of some polymers.

\begin{tabular}{lc}
\hline Polymer & LOI \\
\hline Polypropylene & 18 \\
Poly(butylene terephthalate) & 20 \\
Poly(ethylene terephthalate) & 21 \\
Nylon-6,6 & 24 \\
Nylon-6 & 21 \\
Cotton & 16 \\
Polyester fabric & 21 \\
Wool & 24 \\
Polyacrylonitrile & 18 \\
Polyaramid & 38 \\
\hline
\end{tabular}

Medium-scale experiments, based on oxygen consumption calorimetry, such as the cone calorimetric measurements, are generally considered to generate parameters that are more relevant to real fire scenarios involving polymeric materials [8]. The measured parameters include time to ignition, heat release rate, total heat released, mass loss rate, effective heat of combustion, smoke specific extinction area, smoke production rate and total smoke production (Table 2). The central objective in carrying out the oxygen consumption calorimetric techniques is to obtain parameters that represent the true nature of the fire hazards possessed by the materials under investigation such as the heat release rates (as well as the total heat released) and production of smoke and $\mathrm{CO}$ [9]. The measurements should be carried on sufficiently thick samples to avoid thermally thin behaviour, by and using sample holder with minimum heat losses so as to minimize the effects of the apparatus employed on the values of the parameters obtained. This will ensure that the values collected through such measurements are in turn more global in nature [10].

Table 2. Some representative peak heat release rates obtained by cone calorimetry.

\begin{tabular}{lc}
\hline Polymer & Peak Heat Release Rate ${ }^{\mathbf{a}} \mathbf{( \mathbf { k W ~ m }} \mathbf{~}^{\mathbf{2}} \mathbf{)}$ \\
\hline Polypropylene & 1095 \\
Poly(butylene terephthalate) & 1313 \\
Isophthalic polyester & 985 \\
Nylon-6,6 & 1313 \\
Nylon-6 & 863 \\
Wool & 307 \\
Acrylic fibres & 346 \\
\hline
\end{tabular}

${ }^{\mathrm{a}}$ Measured under an irradiance of $40 \mathrm{~kW} \mathrm{~m}{ }^{-2}$.

Similar parameters, as obtained through a cone calorimeter, can be collected from much lesser quantities of materials ( $c a .5 \mathrm{mg}$ or so), with a Pyrolysis Combustion Flow Calorimeter (PCFC) which is based on the principle of oxygen depletion calorimetry [11]. Here the influences of sample size, thickness and morphological features are essentially eliminated. Accurately weighed solid samples (ca. $5 \mathrm{mg}$ ) are first heated to about $900{ }^{\circ} \mathrm{C}$ at constant heating rate of $1 \mathrm{Ks}^{-1}$, in a stream of nitrogen flowing at a rate of $80 \mathrm{~cm}^{3} \mathrm{~min}^{-1}$. The thermal degradation products, thus obtained, were then mixed with a $20 \mathrm{~cm}^{3} \mathrm{~min}^{-1}$ stream of oxygen prior to entering a combustion chamber maintained at $900{ }^{\circ} \mathrm{C}$. 
Each sample is run in triplicate and the data obtained are averaged over the three measurements [12]. The instrument also generates plots of the Heat Release Rates (HRR) against the temperature and gives values for the maximum amount of heat released per unit mass per degree of temperature (i.e., heat release capacity measured in $\mathrm{Jg}^{-1} \mathrm{~K}^{-1}$ ), the latter being a reliable indicator regarding flammability of a material (Table 3).

Table 3. Heat release capacity, total heat released, and char yield of selected polymers [11].

\begin{tabular}{lccc}
\hline Polymer Char & $\begin{array}{c}\text { Heat Release Capacity } \\
\left(\mathbf{J ~ g}^{-\mathbf{1}} \mathbf{~ K} \mathbf{- 1}\right)\end{array}$ & $\begin{array}{c}\text { Total Heat Released } \\
(\mathbf{k J ~ g} \mathbf{~} \mathbf{)}\end{array}$ & $\begin{array}{c}\text { Residue } \\
(\mathbf{w t . \%})\end{array}$ \\
\hline Polypropylene & 1571 & 41.1 & 0 \\
Polyethylene (LDPE) & 1676 & 41.6 & 0 \\
Polystyrene & 927 & 38.8 & 0 \\
Poly(butyleneterephthalate) & 474 & 20.3 & 1.5 \\
Poly(ethyleneterephthalate) & 332 & 15.3 & 5.1 \\
Polymethylmethacrylate & $376-514$ & 23.2 & 0 \\
Polyoxomethylene & 169 & 10 & 0 \\
Polyvinylchloride & 138 & 11.3 & 15.3 \\
\hline
\end{tabular}

Net heats of combustion of polymeric materials are a good indicator of their combustibility (assuming complete combustion), and therefore a good indicator of the total heat released. Heats of combustion are usually measured using an oxygen bomb calorimetric technique, where the sample is burned in an atmosphere of excess and hyperbaric oxygen. A similar parameter can be also deduced from PCFC runs (Table 4).

Table 4. Net heats of combustion of selected polymers by PCFC and oxygen bomb calorimetry [11].

\begin{tabular}{lcc}
\hline Polymer & \multicolumn{2}{c}{ Net Heat of Combustion $\left(\mathbf{k J ~ g ~}^{\mathbf{- 1}}\right)$} \\
\hline & PCFC & Oxygen Bomb \\
Polyethylene & 44.1 & 43.3 \\
Polystyrene & 40.1 & 39.8 \\
Polycarbonate & 29.1 & 29.8 \\
Poly(butyleneterephthalate) & 26.3 & 26.7 \\
Poly(ethyleneterephthalate) & 23.2 & 21.8 \\
Polymethylmethacrylate & 25.0 & 25.4 \\
Polyoxomethylene & 15.0 & 15.9 \\
\hline
\end{tabular}

\subsection{Combustion of Some Representative Polymeric Solid Fuels}

\subsubsection{Polyolefins}

Polyolefins are among the most important polymers in terms of production volume. By copolymerization of ethylene and propylene with higher $n$-olefins, cyclic olefins, or polar monomers, product properties can be varied considerably, thus extending the range of possible applications. Polyethylenes (PE) are manufactured in the largest tonnage of all thermoplastic materials. Several well-establishes families of polyethylenes are now available on the market, each having a different 
molecular architecture and different behaviour, performance and applications, e.g., low-density polyethylene (LDPE), high-density polyethylene (HDPE), linear low-density polyethylene (LLDPE), very low-density polyethylene (VLDPE), and high and ultra-high molecular weight polyethylenes. The main structural features of that determine the properties of PE are the degrees of short- and long-chain branching, the average molecular weight and polydispersity.

Generally, polyolefins are highly flammable. The principal mechanism of thermal degradation being homolytic chain scission, followed by inter- and intra-molecular chain transfer, resulting in the formation of volatile fragments. The long-chain fragments and the soot-like products formed by cyclization dehydrogenation, contribute to smoke development. Carbon dioxide and water are also formed during combustion. Polyolefins burn readily in air (LOI = 18) with melting and dripping, and produce little or no residual char.

\subsubsection{Acrylics}

Acrylic polymers are obtained from derivatives of acrylic and methacrylic acids; the group includes also their copolymers with various vinylic and allylic monomers. The largest applications of acrylic polymers in terms of tonnages used are in moulded and fabricated plastic articles of many kinds made from poly(methyl methacrylate) (PMMA). On heating PMMA undergoes extensive chain unzipping or depolymerisation to produce quantitative yield (>90\%) of monomers and is, as a consequence, highly flammable $(\mathrm{LOI}=18)$. The oxygen of the ester group assists complete combustion of the pyrolysis products and is the reason for the low smoke production of the burning polymer. The material melts and volatilises so that no residue remains.

\subsubsection{Elastomers}

The common form of polyisoprene is cis-1,4-polyisoprene, which occur in the latex of many plants and trees as natural rubber. The trans isomer can be isolated from some plants as gutta percha or balata. Both these forms, and their derivatives, may also be synthesised by the use of stereospecific catalysts. Generally, natural rubber and other related polydienes such as poly-1,3-butadienes burns readily in air $(\mathrm{LOI}=17)$.

\subsection{Enhancement in Degradation and Regression Rates in Hybrid Fuels—Some Suggestions for}

Future Work

The overriding principle here is to obtain, as fully as possible, the structure property relationships of some novel materials to be tested as components of hybrid fuel systems for rocket propulsion that have enhanced degradation/regression rates as compared to the un-modified counterparts. Synthetic strategies for making these materials could include copolymerization reactions, grafting reactions and other post-chemical modifications on preformed precursor polymers [13-18] (Figure 3). 
Figure 3. Synthetic strategies.
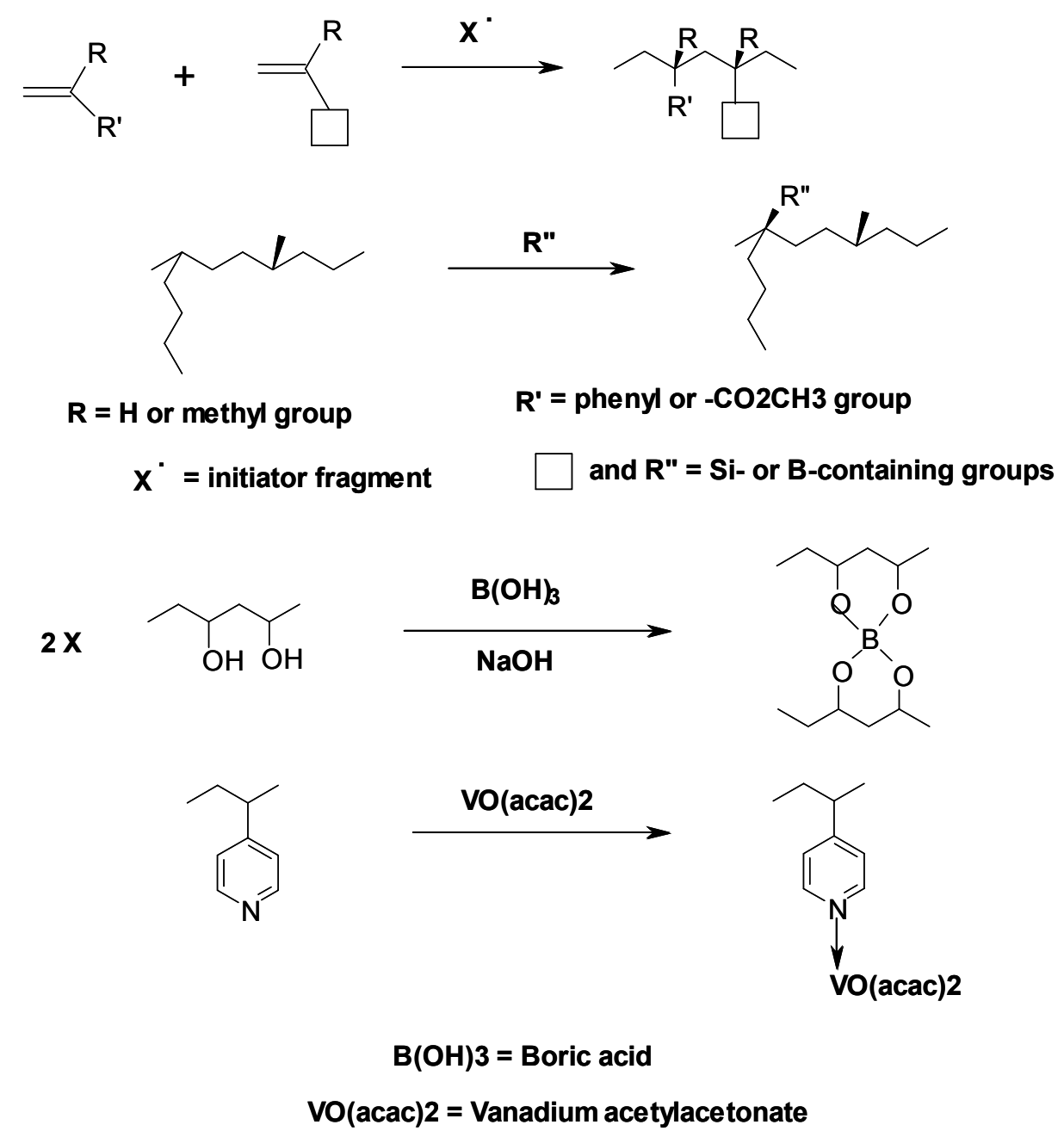

The characterization techniques employed, on recovered and purified materials, could include:

(a) Spectroscopic (NMR and FT-IR) and elemental analyses- high field (500 MHz) solution state ${ }^{1} \mathrm{H}$ - and ${ }^{13} \mathrm{C}$-NMR for deciphering the microstructures of the polymers (this includes tacticity, composition, monomer sequencing, minor structures including structural defects, etc.). Limited, but complementary, information regarding the structural features of the polymer could also be obtained from the FT-IR spectra and heteroatom elemental analyses.

(b) Chromatographic and related techniques - these are primarily aimed at obtaining the molecular weights and their distributions. For polyolefin based-polymers, optionally melt-flow index measurements could be carried out.

(c) Thermo-gravimetric analyses (TGA) - TGA runs need to be carried out on ca. 10-15 mg of the resin in nitrogen, air and in oxygen atmospheres, say, at $10^{\circ} \mathrm{C} \mathrm{min}^{-1}$, and from 30 to $1000{ }^{\circ} \mathrm{C}$. The idea behind these runs is to get the general thermal- and thermo-oxidative degradation profiles of the material (i.e., under different oxidative atmospheres). This could be followed by repeating the runs, in a chosen atmosphere(s), with a view to estimating the Arrhenius parameters, if necessary. 
(d) Differential Scanning Calorimetry (DSC) - here, milligrams of samples are heated in sealed aluminium pans, under a nitrogen atmosphere and usually at a heating rate of $10{ }^{\circ} \mathrm{C} \mathrm{min}^{-1}$, up to a point where substantial thermal degradation starts. This is a very useful technique that often yields information regarding melting behaviours, glass transition temperatures, etc. that the material might undergo under the heating conditions imposed.

(e) Parallel Plate Rheometry-here again the sample, ideally in the shape of thin films, is heated whilst sandwiched between two heated parallel plates, at the same time a sinusoidal mechanical stress is applied. Generally, this constitute a good method for determining the moduli of elasticity (store and loss), the glass transition temperatures, and more importantly the melt flow behaviour of the resin

(f) Combustion Bomb Calorimeter-this instrumentation is used to determine the heats of combustion $\left(\Delta H_{\text {comb }}\right)$ of the resin. This parameter is a good indicator of the maximum heat out put on complete oxidation of the polymeric material in question.

(g) Pyrolysis Combustion Flow Calorimetry (PCFC) - this piece of instrumentation, often dubbed as the micro cone calorimeter, produces plots of Heat Release Rates against time, as well as generates parameters like the heat release capacity on milligrams of a material (i.e., the maximum amount of heat released per unit mass per degree of temperature $\left(\mathrm{Jg}^{-1} \mathrm{~K}^{-1}\right.$, is a material property that appears to be a good predictor of flammability).

(h) Hyphenated techniques - attempts to identify the volatiles formed from thermal degradation of the materials could be made by hyphenating the TGA to an FT-IR or to a GC/MS. Such hyphenated technique is also available in a larger scale that, primarily, involves two consecutive tube furnaces in connected in series. Optionally, some of the gaseous-products formed upon degradation, in ambient atmosphere, collected through by using proprietary containers, will be subjected to GC/MS.

The main areas of interest here are: (a) try to find the influence of the molecular weights (i.e., the chain lengths, or the degree of polymerization), structural defects and other microstructure on the thermal/thermo-oxidation behaviours; (b) gauge the "ease" of thermal/thermo-oxidative degradation, and thus hopefully leading to correlations with the regression rates; (c) estimate the influences of combustion energetics (such as the heats of combustion, the heat release and its rate, residue left), the effects of the heteroatom bearing groups/transition metal ( $\mathrm{Si}$ and $\mathrm{B}$, or V), the morphology, melt-drip behaviours, etc. on the overall performance of the material as a rocket fuel d) carryout further chemical modifications, in the wake of the results obtained through c, with a view to improving the overall performance of the polymeric substrates as components of hybrid rocket engine systems.

\section{CFD Modelling Framework for Hybrid Propulsion}

This section presents general approaches to implementation of comprehensive hybrid rocket combustion model. 


\subsection{Fuel Regression}

Overall steps of polymeric fuel pyrolysis and combustion can be described as follows:

- Thermal decomposition

- Thermo-oxidative decomposition

- Decomposition of monomer MMA

- Combustion of pyrolysis products

In a semi-empirical way, regression is predicted by classical correlations, which are mainly due to Marxman and Gilbert [19] and Marxman et al. [20]. Their basic correlation for the regression rate $\dot{m}$ is presented in the form:

$$
\left.\rho_{f} \dot{m}=C G \operatorname{Re}_{x}^{-0.2}\left(S t / S t_{0}\right)\left(u_{e} / u_{f l}\right)\right)\left[\left(h_{f l}-h_{w}\right) / \Delta H_{s g}^{t o t}\right]
$$

where $C$ is a function of mainstream Mach number, $\operatorname{Re}_{x}$ is local Reynolds number and $G$ is local mass flux due to oxidizer injection and upstream fuel addition. $S t$ and $S t_{0}$ are Stanton numbers for turbulent boundary layer flow in the presence and in the absence of blowing, respectively. Further, $u_{e}$ is the velocity at the edge of the boundary layer, $u_{f l}$ is the velocity at the flame location, $h_{B}$ is stagnation enthalpy at the flame temperature, $h_{w}$ is gas enthalpy at the wall, and $\Delta H_{s g}^{\text {tot }}$ is the total heat of gasification. The latter is the total energy required to heat a unit mass of solid fuel up from its initial temperature and vaporise it.

Further corrections to this correlation include effects of radiation, variable fluid properties, chemical kinetics, ablation and other factors. Comprehensive details of various correlations are given in detail by Chiaverini [21] and Lengelle [22].

The purpose of the present paper is, in contrast, to discuss modelling approaches that will allow prediction of fuel regression behaviour and combustion process from the first principles. Such a modelling framework is discussed in the sections below.

\subsection{Polymer Decomposition Modelling}

Qualitatively, decomposition of common polymers has been relatively well understood. For example, in the thermal decomposition of PMMA there are eight more important elementary mechanisms, arising from the two principal mechanisms: thermal $C-C$ bonds scission, and random $C-C$ scission [23]. Further, up to 15 elementary steps can be identified in thermal oxidative decomposition of PMMA, and up to 25 elementary reactions in monomer (MMA) decomposition [23].

However, despite detailed qualitative understanding of the elementary reactions involved, their specific kinetic parameters are rarely known. Consequently, development of very detailed, multi-step kinetic model of PMMA decomposition seems problematic at the present stage.

Thermal unzipping of PMMA occurs at $\sim 200{ }^{\circ} \mathrm{C}$, so that polymer is decomposed into monomer. This is accompanied by formation of viscous layer of melt polymer at the surface. Formed monomer bubbles through this viscous layer and escapes into the gas phase. Thickness of viscous layer may be negligible under high heat fluxes, but will grow as heat flux to the surface decreases. Therefore, generally, modelling is expected to be more challenging under low heat flux conditions. 
It is seen from this qualitative description that the process of thermal decomposition is fairly complicated and its modelling is far from being straightforward.

One can envisage "macro-" and "micro-" approaches to modelling. In a macro-approach, combined effect of all surface processes (physical and chemical) will be effectively correlated with few key parameters describing decomposition. Under such approach, thermal decomposition can, in principal, be described via specifying melt temperature and considering evaporation process using Clausius-Clapeyron relation. However, some deviations from Clausius-Clapeyron relation during decomposition of PMMA are reported in a literature. Therefore, the simpler and better way will be to assume decomposition rate to obey Arrhenius kinetics, and fit the kinetic parameters from pyrolysis tests.

This leads to kinetic equation of the form:

$$
\dot{m}_{p}=A_{p} \exp \left(-E_{p} / R T_{s}\right)
$$

for the mass decomposition rate.

A significant amount of work has been devoted to fitting the constants from experimental data for a number of polymer materials. Many of these studies have been driven by fire modelling applications. Representative values (for example, due to Ananth et al. [24] for PMMA) are as follows:

Table 5. Estimated kinetic properties of PMMA.

\begin{tabular}{lll}
\hline$A_{p}$ & pyrolysis pre-exponential factor, $\mathrm{kg} /\left(\mathrm{m}^{2} \mathrm{~s}\right)$ & $\sim 8 \times 10^{17}$ \\
$E_{p}$ & pyrolysis activation energy, $\mathrm{J} / \mathrm{mol}$ & $\sim 2.8 \times 10^{5}$ \\
\hline
\end{tabular}

Similar estimation attempts have been carried out by Stoliarov et al. [25], Arisawa and Brill [26], Bedir and T'ien [27].

Typical procedure for measurement of kinetic parameters involves relevant equipment, for example, Mettler Toledo TGA/SDTA851e thermogravimetric analyzer. Stoliarov et al. [25] used heating rates of $0.05 \mathrm{~K} \mathrm{~s}^{-1}, 0.17 \mathrm{~K} \mathrm{~s}^{-1}$, and $0.5 \mathrm{~K} \mathrm{~s}^{-1}$ to bring polymer sample from $373 \mathrm{~K}$ to $1003 \mathrm{~K}$. Experiments were conducted in a nitrogen atmosphere.

If decomposition is assumed to be a first order reaction, and reaction rate constants $k_{D}$ are calculated by numerical differentiation of mass loss data, then the rate constants may be plotted against temperature. Assuming:

$$
k_{D}=A \exp \left(-E_{p} / R T_{s}\right)
$$

fit of kinetic constants can be made to produce the following results (Table 6) for selected polymers (Stoliarov et al. [25]). Here $h_{D}$ and $h_{C}$ are the heats of decomposition reactions and the heats of combustion of volatile decomposition products, respectively.

Unfortunately, the data available in literature show significant scatter. This could be attributed to the inhomogeneities arising due the multi-phases and multi-components involved in the degradation profiles of polymeric substances during thermo-gravimetric runs. Furthermore the profiles, themselves, could be influenced by the morphology of the sample as well by the heating rates employed. Therefore, evaluation of Arrhenius parameters from thermo-gravimetric data, especially, under isothermal conditions has been met with severe criticism. Arisawa and Brill [26] find the value of $2.76 \times 10^{5} \mathrm{~J} / \mathrm{mol}$ for the activation energy. 
Table 6. Kinetic and thermal properties of selected polymers.

\begin{tabular}{lllll}
\hline Polymer & $\begin{array}{l}\boldsymbol{E} \\
\left(\mathbf{s}^{\mathbf{- 1}}\right)\end{array}$ & $\begin{array}{l}\boldsymbol{h}_{\boldsymbol{D}} \\
\left(\mathbf{J ~ m o l} \mathbf{~}^{-\mathbf{}}\right)\end{array}$ & $\begin{array}{l}\boldsymbol{h}_{\boldsymbol{C}} \\
\left(\mathbf{J ~ k g} \mathbf{~ k g}^{-\mathbf{1}}\right)\end{array}$ \\
\hline PMMA & $8.5 \times 10^{12}$ & $1.88 \times 10^{5}$ & $8.7 \times 10^{5}$ & $2.41 \times 10^{7}$ \\
HIPS & $1.2 \times 10^{16}$ & $2.47 \times 10^{5}$ & $1.0 \times 10^{6}$ & $3.81 \times 10^{7}$ \\
HDPE & $4.8 \times 10^{22}$ & $3.49 \times 10^{5}$ & $9.2 \times 10^{5}$ & $4.35 \times 10^{7}$ \\
\hline
\end{tabular}

Significantly lower values are reported by Vovelle et al. [28], and by Krishnamurthy and Williams [29]. Kashiwagi and and Brown [30] showed that the activation energy is generally much larger $\left(2.35 \times 10^{5} \mathrm{~J} / \mathrm{mol}\right)$ in the absence of oxygen at the PMMA surface than that $\left(6.3 \times 10^{4} \mathrm{~J} / \mathrm{mol}\right)$ for oxidative degradation.

Since there is significant scattering in kinetic data for a global reaction, it is very desirable to conduct independent series of tests to confirm values of pre-exponential factor and activation energy. Further, it seems likely that for more accurate models, these parameters should be treated as temperature-dependent. Data for other polymers is less readily available. The most studied of them are hydroxyl-terminated polybutadiene (HTPB) polymers group. Table 7 reproduces Arrhenius data from Arisawa and Brill [26].

Table 7. Arrhenius Data for Polybutadiene Polymers at Heating Rates $\leq 100{ }^{\circ} \mathrm{C} / \mathrm{min}$.

\begin{tabular}{|c|c|c|c|c|}
\hline$E_{a}(\mathrm{kcal} / \mathrm{mol})$ & $\ln A\left(s^{-1}\right)$ & $T\left({ }^{\circ} \mathrm{C}\right)$ & Ref. & Comments \\
\hline $31 \pm 3^{a}$ & - & cis-trans Isomerization 200-300 & {$[3]^{a}$} & IR, vacuum \\
\hline $15^{a}$ & - & $\begin{array}{l}\text { Cross-linking and Cyclization } \\
200-300\end{array}$ & {$[9]$} & $\begin{array}{l}\text { IR, vacuum, first order, } \\
\text { vinyl groups }\end{array}$ \\
\hline $18.8^{b}$ & 2.5 & $328-420$ & {$[4]$} & TGA \\
\hline $27.6 \pm 1.6^{b}$ & 16.2 & $350-400$ & {$[13]^{b}$} & TGA \\
\hline 39 & - & 250 & {$[11]$} & Hardening data \\
\hline $37.6^{c}$ & 25.6 & $\begin{array}{l}\text { Chain Scission } \\
450-532\end{array}$ & {$[15]$} & $\begin{array}{l}\text { pyrolysis-GC, } \mathrm{BD} \\
\text { formation, first order }\end{array}$ \\
\hline $40.7 \pm 3$ & - & N/A & {$[10]$} & $\begin{array}{l}\text { estimate of 4-vinyl-1 } \\
\text { cyclohexene formation }\end{array}$ \\
\hline $42.1^{a}$ & - & $362-434$ & {$[10]$} & $\mathrm{DSC}$ \\
\hline 42.8 & - & - & {$[16]$} & Estimate \\
\hline $46 \pm 0.6^{b}$ & 22.8 & $436-470$ & {$[4]$} & TGA \\
\hline $51.9^{a}$ & & $350-425$ & {$[10]$} & TGA \\
\hline $60.0 \pm 3.5^{a}$ & - & N/A & {$[10]$} & $\begin{array}{l}\text { Estimate of BD } \\
\text { formation }\end{array}$ \\
\hline $60.1^{b}$ & 17.1 & $367-407$ & {$[13]$} & TGA \\
\hline 62 & - & $380-395$ & {$[14]$} & weight change \\
\hline $62 \pm 4^{d}$ & - & 410-500 Unspecified Processes & {$[6]$} & TGA \\
\hline $24.5-38.5^{e}$ & $12.2-20.8$ & $350-550$ & {$[7]$} & TGA \\
\hline $28^{e}$ & 12.8 & $400-500$ & {$[18]$} & TGA, order $=0.6-1$ \\
\hline $21.5-31.1^{b}$ & $8.9-13.8$ & N/A & {$[19]$} & TGA \\
\hline
\end{tabular}


Micro-approach ultimately aims at describing the process from molecular dynamical point of view. Examples of approaching this level of description can be found in the work by Kumar and Stickler [31]. They consider the energy and polymer backbone bond conservation equations. The approach provides more detailed treatment, considering details of polymer fragmentation and pressure sensitivity.

The model in this approach is developed as follows. It is assumed that for a given initial solid temperature the two parameters that control the regression rate are the polymer fragment size characteristic of the degradation [Fragment Size Vaporizing (FSV)], and the polymer surface temperature. The fractional number of backbone bonds changes from unity in the cold material to some non-zero value at the surface. Polymer pieces that leave the surface and enter the gas phase have different fragment sizes. Mean fragment size specifies the number of backbone bonds still left and thus serves as the required boundary condition for the fractional bond number. One way of implementing this boundary condition is the requirement that vapor pressure of FSV at the surface temperature is equal to the chamber pressure. A noninteger value of FSV is interpreted as a weighted mean value of all fragment sizes that are actually coming off the surface. The vapor-pressure boundary condition assumes quasiequilibrium between the surface phase and the vapor phase, and also that only the products of depolymerization contribute to pressure at the interface. Following Madorsky [32], global depolymerisation reaction is reproduced by first order Arrhenius kinetics. Consequently, the governing equations are written for the energy conservation and bond conservation. The latter contains source term with Arrhenius dependence. Obtained numerical solutions demonstrated good agreement with experimental data for inert atmospheres.

In general, micro-approach is still rather under-developed, and taking into account overall uncertainties and modelling limitations in Hybrid Rocket combustions, it is doubtful that such approach can be really helpful in the immediate future.

In addition to thermal decomposition, material also undergoes thermal oxidative decomposition, caused by direct interaction of oxygen molecules with the surface. This type of decomposition becomes increasingly important in high-oxygen concentration environments. The actual mode of attack of the biradical centred oxygen molecule on to the polymer chain is largely influenced by the chemical constitution of the repeating units in it. For example, tertiary hydrogens are particularly amenable to the reaction with oxygen, thus producing hydroperoxide structures. This, in turn, could trigger other chemical pathways to produce ketonic and other oxygenated attributes onto the chain structure. Generally, the above reactions contribute to centres of weak links that would eventually facilitate further degradation. Overall, available data suggest that additional tests are desirable to enhance kinetic parameters reliability. Development of micro-scale depolymerisation models may be beneficial as medium- to long term goals.

\subsection{Polymer Combustion Modelling}

\subsubsection{Global Kinetics}

In a very simple form, gaseous combustion can be modelled in the same way as thermal decomposition, i.e., with one step global reaction approach. 
For complete oxidation, example of such a reaction is given by (PMMA):

$$
\mathrm{C}_{5} \mathrm{H}_{8} \mathrm{O}_{2}+6 \mathrm{O}_{2} \rightarrow 5 \mathrm{CO}_{2}+4 \mathrm{H}_{2} \mathrm{O}
$$

Kinetic data reported in a literature show considerable discrepancy. Representative kinetic parameters are given, for example, by Bedir and T'ien [27], who assume absolute value of reaction rate for the $i$-th species in the form:

$$
\dot{\omega}_{i}=B T \frac{r_{i}}{\mu_{O}} \rho^{2} Y_{F} Y_{O} \exp \left(-E_{c} / R T\right)
$$

with the kinetic data given in:

Table 8. Kinetic data for global combustion reaction of PMMA.

\begin{tabular}{lll}
\hline$B$ & combustion pre-exponential factor, $\mathrm{m}^{3} /(\mathrm{s} \mathrm{mol} \mathrm{K})$ & $\sim 6.6 \times 10^{6}$ \\
$E_{c}$ & combustion activation energy, $\mathrm{J} / \mathrm{mol}$ & $\sim 1.44 \times 10^{5}$ \\
\hline
\end{tabular}

where $r_{i}$ is a stoichiometric requirement of the $i$-th species with respect to fuel. Again, as for decomposition, additional independent tests are desirable, and temperature-dependent treatment of parameters may be appropriate.Global combustion reaction is quite a crude approach, so priority should be given to developing more accurate models incorporating detailed chemistry.

\subsubsection{Detailed Kinetics}

The polymer structure of Polymethylmethacrylate (PMMA) (as a representative polymer fuel) is presented in Figure 4.

Figure 4. Structure of PMMA.

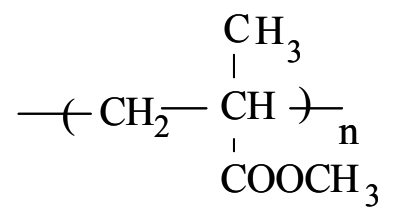

Upon decomposition, gaseous molecules of various species, including combustible gases, are formed. Generally, there is a good qualitative understanding of this process. The major combustibles that are formed are methane, methanol, formaldehyde, and acetylene [23,33]. Detailed kinetic schemes and parameters for methane can be found, for example, using the well-known GRI-MECH database [34]. Examples of detailed kinetic mechanisms for the other components are presented in Tables 9-11 [23,33].

Table 9. Methanol combustion mechanisms proposed by Bell and Tipper [35].

\begin{tabular}{ll}
\hline $\mathrm{CH}_{3} \mathrm{OH}+\mathrm{O}_{2} \rightarrow \mathrm{CH}_{2} \mathrm{OH}+\mathrm{HO}_{2}$ & $\mathrm{CHO}+\mathrm{O}_{2} \rightarrow \mathrm{CO}+\mathrm{HO}_{2}$ \\
$\mathrm{CH}_{3} \mathrm{OH}+\mathrm{HO}_{2} \rightarrow \mathrm{CH}_{2} \mathrm{OH}+\mathrm{H}_{2} \mathrm{O}_{2}$ & $\mathrm{CH}_{2} \mathrm{O}+\mathrm{HO}_{2} \rightarrow \mathrm{CHO}+\mathrm{H}_{2} \mathrm{O}_{2}$ \\
$\mathrm{CH}_{2} \mathrm{OH}+\mathrm{O}_{2} \rightarrow \mathrm{CH}_{2} \mathrm{O}+\mathrm{HO}_{2}$ & $\mathrm{HO}_{2}+\mathrm{O}_{2} \rightarrow$ inner products \\
$\mathrm{CH}_{2} \mathrm{O}+\mathrm{O}_{2} \rightarrow \mathrm{CHO}+\mathrm{HO}_{2}$ & \\
\hline
\end{tabular}


Table 10. Formaldehyde combustion mechanisms proposed by Vardanyan et al. [36], Hay and Hessam [37].

\begin{tabular}{ll}
\hline $\mathrm{HCHO}+\mathrm{O}_{2} \rightarrow \mathrm{HO}_{2}+\mathrm{CO}$ & $\mathrm{HCO}_{3} \mathrm{H} \rightarrow \mathrm{OH}+$ Products \\
$\mathrm{HCHO}+\mathrm{O}_{2} \rightarrow \mathrm{OH}+$ Products & $\mathrm{HO}_{2}+\mathrm{HCHO} \rightarrow \mathrm{H}_{2} \mathrm{O}_{2}+\mathrm{HCO}$ \\
(HCOOH or $\left.\mathrm{H}_{2}+\mathrm{OO}+\mathrm{H}_{2} \mathrm{O}\right)$ & \\
$\mathrm{HCHO}+\mathrm{OH} \rightarrow \mathrm{H}_{2} \mathrm{O}+\mathrm{HOO}$ & $\mathrm{HO}_{2}+\mathrm{HO}_{2} \rightarrow \mathrm{H}_{2} \mathrm{O}_{2}$ \\
$\mathrm{HOO}+\mathrm{M} \rightarrow \mathrm{H}+\mathrm{OO}+\mathrm{M}$ & $\mathrm{H}_{2} \mathrm{O}_{2} \rightarrow \mathrm{HO}_{2}+1 / 2 \mathrm{O}_{2}$ \\
$\mathrm{HOO}+\mathrm{O}_{2} \rightarrow \mathrm{HO}_{2}+\mathrm{OO}$ & $\mathrm{H}_{2} \mathrm{O}_{2}+\mathrm{M} \rightarrow 2 \mathrm{OH}+\mathrm{M}$ \\
$\mathrm{HOO}+\mathrm{O}_{2} \rightarrow \mathrm{HCO}_{3}$ & $\mathrm{HO}_{2} \rightarrow 1 / 2 \mathrm{H}_{2} \mathrm{O}+3 / 4 \mathrm{O}_{2}$ \\
$\mathrm{HCO}_{3}+\mathrm{HCHO} \rightarrow \mathrm{HCO}_{3} \mathrm{H}+\mathrm{HCO}$ & \\
\hline
\end{tabular}

Table 11. Acetylene combustion mechanisms proposed by Hidaka et al. [38].

\begin{tabular}{|c|c|}
\hline $\mathrm{C}_{2} \mathrm{H}_{2}+\mathrm{M} \mathrm{C}_{2} \mathrm{H}+\mathrm{H}+\mathrm{M}$ & $\mathrm{CH}_{2}+\mathrm{CH}_{2} \mathrm{C}_{2} \mathrm{H}_{4}+\mathrm{H}$ \\
\hline $\mathrm{C}_{2} \mathrm{H}_{2}+\mathrm{C}_{2} \mathrm{H}_{2} \mathrm{C}_{4} \mathrm{H}_{2}+\mathrm{H}$ & $\mathrm{CH}_{2}+\mathrm{CH}_{2} \mathrm{C}_{2} \mathrm{H}_{4}+\mathrm{H}_{2}$ \\
\hline $\mathrm{C}_{2} \mathrm{H}_{2}+\mathrm{O}_{2} \mathrm{CHO}+\mathrm{CHO}$ & $\mathrm{CH}_{2}+\mathrm{CH}_{2} \mathrm{C}_{2} \mathrm{H}_{5}+\mathrm{H}$ \\
\hline $\mathrm{C}_{2} \mathrm{H}_{2}+\mathrm{H} \mathrm{C}_{2} \mathrm{H}+\mathrm{H}_{2}$ & $\mathrm{CH}_{2}+\mathrm{HCH}+\mathrm{H}_{2}$ \\
\hline $\mathrm{C}_{2} \mathrm{H}_{2}+\mathrm{O} \mathrm{CH}_{2}+\mathrm{CO}$ & $\mathrm{CH}_{2}+\mathrm{O}_{2} \mathrm{CO}_{2}+\mathrm{H}+\mathrm{H}$ \\
\hline $\mathrm{C}_{2} \mathrm{H}_{2}+\mathrm{O} \mathrm{CHCO}+\mathrm{H}$ & $\mathrm{CH}_{2}+\mathrm{CH}_{2} \mathrm{C}_{2} \mathrm{H}_{2}+\mathrm{H}+\mathrm{H}$ \\
\hline $\mathrm{C}_{2} \mathrm{H}_{2}+\mathrm{CH} \mathrm{C} \mathrm{C}_{2} \mathrm{H}+\mathrm{H}_{2} \mathrm{O}$ & $\mathrm{C}_{2} \mathrm{H}_{6} \mathrm{CH}_{2}+\mathrm{CH}_{2}$ \\
\hline $\mathrm{C}_{2} \mathrm{H}_{2}+\mathrm{CH} \mathrm{CH}{ }_{2} \mathrm{CO}+\mathrm{H}$ & $\mathrm{C}_{2} \mathrm{H}_{6}+\mathrm{H} \mathrm{C}_{2} \mathrm{H}_{5}+\mathrm{H}_{2}$ \\
\hline $\mathrm{C}_{2} \mathrm{H}_{2}+\mathrm{CH} \mathrm{C}_{2} \mathrm{H}_{2}$ & $\mathrm{C}_{2} \mathrm{H}_{6}+\mathrm{CH} \mathrm{C}_{2} \mathrm{H}_{5}+\mathrm{H}_{2}$ \\
\hline $\mathrm{C}_{2} \mathrm{H}_{2}+\mathrm{CH}_{2} \mathrm{C}_{2} \mathrm{H}_{4}$ & $\mathrm{C}_{2} \mathrm{H}_{6}+\mathrm{CH}_{2} \mathrm{CH}_{4}+\mathrm{C}_{2} \mathrm{H}_{5}$ \\
\hline $\mathrm{C}_{2} \mathrm{H}_{2}+\mathrm{CH}_{2} \mathrm{C}_{2} \mathrm{H}+\mathrm{CH}_{4}$ & $\mathrm{C}_{2} \mathrm{H}_{5} \mathrm{C}_{2} \mathrm{H}_{4}+\mathrm{H}$ \\
\hline $\mathrm{C}_{2} \mathrm{H}_{2}+\mathrm{C}_{2} \mathrm{H} \mathrm{C}_{4} \mathrm{H}_{2}+\mathrm{H}$ & $\mathrm{C}_{2} \mathrm{H}_{4}+\mathrm{M} \mathrm{C}_{2} \mathrm{H}_{2}+\mathrm{H}_{2}+\mathrm{M}$ \\
\hline $\mathrm{C}_{2} \mathrm{H}_{2}+\mathrm{C}_{2} \mathrm{H}_{2} \mathrm{C}_{4} \mathrm{H}_{4}+\mathrm{H}$ & $\mathrm{C}_{2} \mathrm{H}_{4}+\mathrm{H} \mathrm{C}_{2} \mathrm{H}_{2}+\mathrm{H}_{2}$ \\
\hline $\mathrm{C}_{2} \mathrm{H}+\mathrm{O}_{2} \mathrm{CO}+\mathrm{CO}+\mathrm{H}$ & $\mathrm{C}_{2} \mathrm{H}_{4}+\mathrm{OCH}_{2}+\mathrm{CHO}$ \\
\hline $\mathrm{CH}_{2} \mathrm{CO}+\mathrm{M} \mathrm{CO}+\mathrm{CH}_{2}+\mathrm{M}$ & $\mathrm{C}_{2} \mathrm{H}_{4}+\mathrm{CH} \mathrm{C}_{2} \mathrm{H}_{2}+\mathrm{H}_{2} \mathrm{O}$ \\
\hline $\mathrm{CH}_{2} \mathrm{CO}+\mathrm{H} \mathrm{CHCO}+\mathrm{H}_{2}$ & $\mathrm{C}_{2} \mathrm{H}_{2}+\mathrm{M} \mathrm{C}_{2} \mathrm{H}_{2}+\mathrm{H}+\mathrm{M}$ \\
\hline $\mathrm{CH}_{2} \mathrm{CO}+\mathrm{H} \mathrm{CH}_{2}+\mathrm{CO}$ & $\mathrm{C}_{2} \mathrm{H}_{2}+\mathrm{H} \mathrm{C}_{2} \mathrm{H}_{2}+\mathrm{H}_{2}$ \\
\hline $\mathrm{CH}_{2} \mathrm{CO}+\mathrm{O} \mathrm{CH}_{2}+\mathrm{CO}_{2}$ & $\mathrm{C}_{2} \mathrm{H}_{2}+\mathrm{OCH}_{2} \mathrm{CO}+\mathrm{H}$ \\
\hline $\mathrm{CH}_{2} \mathrm{CO}+\mathrm{O} \mathrm{CH}_{2} \mathrm{O}+\mathrm{CO}$ & $\mathrm{C}_{2} \mathrm{H}_{2}+\mathrm{O}_{2} \mathrm{C}_{2} \mathrm{H}_{2}+\mathrm{HO}_{2}$ \\
\hline $\mathrm{CH}_{2} \mathrm{CO}+\mathrm{CH} \mathrm{CH}_{2} \mathrm{O}+\mathrm{CHO}$ & $\mathrm{C}_{2} \mathrm{H}_{2}+\mathrm{O}_{2} \mathrm{CH}_{2} \mathrm{O}+\mathrm{CO}+\mathrm{H}$ \\
\hline $\mathrm{CH}_{2} \mathrm{CO}+\mathrm{CH} \mathrm{CH}{ }_{2} \mathrm{CH}+\mathrm{CO}$ & $\mathrm{C}_{2} \mathrm{H}_{2}+\mathrm{CH}_{2} \mathrm{C}_{2} \mathrm{H}_{2}+\mathrm{CH}_{4}$ \\
\hline $\mathrm{CH}_{2} \mathrm{CO}+\mathrm{CH}_{2} \mathrm{C}_{2} \mathrm{H}_{4}+\mathrm{CO}$ & $\mathrm{C}_{2} \mathrm{H}_{4}+\mathrm{M} \mathrm{C}_{2} \mathrm{H}_{2}+\mathrm{H}+\mathrm{M}$ \\
\hline $\mathrm{CH}_{2} \mathrm{CO}+\mathrm{CH}_{2} \mathrm{CHCO}+\mathrm{CH}_{3}$ & $\mathrm{C}_{2} \mathrm{H}_{4}+\mathrm{H} \mathrm{C}_{2} \mathrm{H}_{2}+\mathrm{H}_{2}$ \\
\hline $\mathrm{CH}_{2} \mathrm{CO}+\mathrm{CH}_{2} \mathrm{C}_{2} \mathrm{H}_{5}+\mathrm{CO}$ & $\mathrm{C}_{2} \mathrm{H}_{4}+\mathrm{H} \mathrm{C}_{2} \mathrm{H}_{2}+\mathrm{CH}_{2}$ \\
\hline $\mathrm{CH}_{2} \mathrm{CO}+\mathrm{CH}_{2} \mathrm{CHCO}+\mathrm{CH}_{4}$ & $\mathrm{C}_{2} \mathrm{H}_{4}+\mathrm{CH}_{2} \mathrm{C}_{2} \mathrm{H}_{2}+\mathrm{CH}_{4}$ \\
\hline $\mathrm{CHCO}+\mathrm{O} \mathrm{CO}+\mathrm{CO}+\mathrm{H}$ & $\mathrm{C}_{4} \mathrm{H}_{4} \mathrm{CH}_{2} \mathrm{CO}+\mathrm{CH}_{2}$ \\
\hline $\mathrm{CHCO}+\mathrm{OH} \mathrm{CO}+\mathrm{CO}+\mathrm{H}_{2}$ & $\mathrm{C}_{4} \mathrm{H}_{4} \mathrm{C}_{4} \mathrm{H}_{2}+\mathrm{H}$ \\
\hline $\mathrm{CHCO}+\mathrm{O}_{2} \mathrm{CO}+\mathrm{CO}+\mathrm{CH}$ & $\mathrm{C}_{4} \mathrm{H}_{4} \mathrm{C}_{2} \mathrm{H}_{2}+\mathrm{C}_{2} \mathrm{H}_{2}$ \\
\hline $\mathrm{CHCO}+\mathrm{H} \mathrm{CH}_{2}+\mathrm{CO}$ & $\mathrm{C}_{4} \mathrm{H}_{4} \mathrm{C}_{4} \mathrm{H}_{2}+\mathrm{H}_{2}$ \\
\hline $\mathrm{CHCO}+\mathrm{CH}_{2} \mathrm{C}_{2} \mathrm{H}_{2}+\mathrm{CO}$ & $\mathrm{C}_{4} \mathrm{H}_{4}+\mathrm{H} \mathrm{C}_{4} \mathrm{H}_{2}+\mathrm{H}_{2}$ \\
\hline $\mathrm{CHCO}+\mathrm{CH}_{2} \mathrm{C}_{2} \mathrm{H}_{4}+\mathrm{CO}$ & $\mathrm{C}_{4} \mathrm{H}_{4}+\mathrm{H} \mathrm{C}_{4} \mathrm{H}_{2}+\mathrm{H}_{2}$ \\
\hline $\mathrm{CHCO}+\mathrm{CHCO} \mathrm{CO}+\mathrm{CO}+\mathrm{C}_{2} \mathrm{H}_{2}$ & $\mathrm{C}_{4} \mathrm{H}_{2}+\mathrm{M} \mathrm{C}_{4} \mathrm{H}_{2}+\mathrm{H}+\mathrm{M}$ \\
\hline $\mathrm{CH}_{2} \mathrm{O}+\mathrm{HCHO}+\mathrm{H}_{2}$ & $\mathrm{C}_{4} \mathrm{H}_{2}+\mathrm{H} \mathrm{C}_{4} \mathrm{H}_{2}+\mathrm{H}_{2}$ \\
\hline
\end{tabular}


Table 11. Cont.

\begin{tabular}{|c|c|}
\hline $\mathrm{CH}_{2} \mathrm{O}+\mathrm{CH} \mathrm{CHO}+\mathrm{H}_{2} \mathrm{O}$ & $\mathrm{C}_{4} \mathrm{H}_{2}+\mathrm{C}_{2} \mathrm{H} \mathrm{C}_{2} \mathrm{H}_{2}+\mathrm{H}$ \\
\hline $\mathrm{CHO}+\mathrm{MCO}+\mathrm{H}+\mathrm{M}$ & $\mathrm{O}_{2}+\mathrm{HCH}+\mathrm{O}$ \\
\hline $\mathrm{CHO}+\mathrm{H} \mathrm{H}_{2}+\mathrm{CO}$ & $\mathrm{H}_{2}+\mathrm{O} \mathrm{CH}+\mathrm{H}$ \\
\hline $\mathrm{CHO}+\mathrm{O}_{2} \mathrm{HO}_{2}+\mathrm{CO}$ & $\mathrm{H}_{2} \mathrm{O}+\mathrm{HCH}+\mathrm{H}_{2}$ \\
\hline $\mathrm{CH}_{2} \mathrm{O}+\mathrm{M} \mathrm{CH}_{2} \mathrm{O}+\mathrm{H}+\mathrm{M}$ & $\mathrm{H}+\mathrm{O}_{2}+\mathrm{MHO}_{2}+\mathrm{M}$ \\
\hline $\mathrm{CH}_{2} \mathrm{OH}+\mathrm{M} \mathrm{CH}_{2} \mathrm{O}+\mathrm{H}+\mathrm{M}$ & $\mathrm{HO}_{2}+\mathrm{H} \mathrm{H}_{2}+\mathrm{O}_{2}$ \\
\hline $\mathrm{CO}+\mathrm{CHCO}+\mathrm{H}$ & $\mathrm{HO}_{2}+\mathrm{HCH}+\mathrm{OH}$ \\
\hline $\mathrm{CH}_{4}+\mathrm{MCH}_{2}+\mathrm{H}+\mathrm{M}$ & $\mathrm{N}_{2} \mathrm{O}+\mathrm{M} \mathrm{N}_{2}+\mathrm{O}+\mathrm{M}$ \\
\hline $\mathrm{CH}_{4}+\mathrm{H} \mathrm{CH}_{2}+\mathrm{H}_{2}$ & $\mathrm{~N}_{2} \mathrm{O}+\mathrm{O} \mathrm{N} \mathrm{N}_{2}+\mathrm{O}_{2}$ \\
\hline $\mathrm{CH}_{4}+\mathrm{O} \mathrm{CH}_{2}+\mathrm{OH}$ & $\mathrm{N}_{2} \mathrm{O}+\mathrm{O} \mathrm{NO}+\mathrm{NO}$ \\
\hline $\mathrm{CH}_{4}+\mathrm{CH} \mathrm{CH}_{2}+\mathrm{H}_{2} \mathrm{O}$ & $\mathrm{N}_{2} \mathrm{O}+\mathrm{H} \mathrm{N}_{2}+\mathrm{OH}$ \\
\hline $\mathrm{CH}_{4}+\mathrm{CH}_{2} \mathrm{CH}_{2}+\mathrm{CH}_{2}$ & $\mathrm{~N}_{2} \mathrm{O}+\mathrm{CH}_{2} \mathrm{CH}_{2} \mathrm{O}+\mathrm{N}_{2}$ \\
\hline $\mathrm{CH}_{2}+\mathrm{H} \mathrm{CH}_{2}+\mathrm{H}_{2}$ & $\mathrm{~N}_{2} \mathrm{O}+\mathrm{CH}_{2} \mathrm{CH}_{2} \mathrm{O}+\mathrm{N}_{2}$ \\
\hline $\mathrm{CH}_{2}+\mathrm{OCH}_{2} \mathrm{O}+\mathrm{H}$ & $\mathrm{N}_{2} \mathrm{O}+\mathrm{C}_{2} \mathrm{H}_{2} \mathrm{CH}_{2} \mathrm{CHO}+\mathrm{N}_{2}$ \\
\hline $\mathrm{CH}_{2}+\mathrm{CH} \mathrm{CH}_{2} \mathrm{O}+\mathrm{H}_{2}$ & $\mathrm{~N}_{2} \mathrm{O}+\mathrm{CO} \mathrm{CO}{ }_{2}+\mathrm{N}_{2}$ \\
\hline $\mathrm{CH}_{2}+\mathrm{CH} \mathrm{CH}_{2} \mathrm{CH}+\mathrm{H}$ & $\mathrm{N}_{2} \mathrm{O}+\mathrm{CHO} \mathrm{CO} \mathrm{CO}_{2}+\mathrm{H}+\mathrm{N}_{2}$ \\
\hline $\mathrm{CH}_{2}+\mathrm{O}_{2} \mathrm{CH}_{2} \mathrm{O}+\mathrm{O}$ & $\mathrm{N}_{2} \mathrm{O}+\mathrm{CHCO} \mathrm{CO}+\mathrm{CHO}+\mathrm{N}_{2}$ \\
\hline $\mathrm{CH}_{2}+\mathrm{HO}_{2} \mathrm{CH}_{2} \mathrm{O}+\mathrm{CH}$ & $\mathrm{N}_{2} \mathrm{O}+\mathrm{C}_{2} \mathrm{H}_{2} \mathrm{CHCO}+\mathrm{H}+\mathrm{N}_{2}$ \\
\hline
\end{tabular}

The major problem lies, however, in obtaining exact composition of pyrolysis gases. The present data is inconclusive in this respect, and it is clear that composition may change depending on the magnitude of heat flux experienced by fuel, and other conditions (for example, pressure). Indicative composition of combustion products are presented in the following Table 12.

Table 12. The content of combustion products of PMMA and MMA in air and Ar at different temperatures [33].

\begin{tabular}{lcccccccc}
\hline \multirow{2}{*}{ Product } & \multicolumn{9}{c}{ Content v/v \% } \\
\cline { 2 - 9 } & \multicolumn{2}{c}{ PMMA (air) } & \multicolumn{3}{c}{ MMA (Ar) } & \multicolumn{3}{c}{ MMA (air) } \\
\cline { 2 - 9 } & $300{ }^{\circ} \mathrm{C}$ & $500{ }^{\circ} \mathrm{C}$ & $300{ }^{\circ} \mathrm{C}$ & $400{ }^{\circ} \mathrm{C}$ & $500{ }^{\circ} \mathrm{C}$ & $300{ }^{\circ} \mathrm{C}$ & $400{ }^{\circ} \mathrm{C}$ & $500{ }^{\circ} \mathrm{C}$ \\
\hline $\mathrm{MMA}$ & 95.5 & 78.9 & 92.7 & 83.8 & 74.2 & 91.6 & 79.8 & 68.2 \\
$\mathrm{CH}_{4}$ & 0.8 & 1.3 & 0.6 & 1.5 & 2.6 & 0.5 & 1.2 & 2.2 \\
$\mathrm{CH}_{2}-\mathrm{CHCH}_{3}$ & & 1.7 & 1.1 & 2.3 & 3.4 & 1.0 & 1.4 & 2.9 \\
$\mathrm{CH}_{2}-\mathrm{C}\left(\mathrm{CH}_{3}\right)_{2}$ & & 1.9 & 1.0 & 2.4 & 3.8 & 1.2 & 1.8 & 3.0 \\
$\mathrm{CH}_{3} \mathrm{OH}$ & 1.8 & 3.2 & 1.6 & 3.6 & 5.8 & 1.5 & 2.9 & 4.9 \\
$\mathrm{HCHO}$ & & 0.3 & & & & & 0.5 & 0.8 \\
$\mathrm{CH}_{3} \mathrm{COCH}$ & & 0.6 & 0.2 & 0.5 & 1.0 & 0.8 & 1.3 & 1.6 \\
$\mathrm{CH}_{3} \mathrm{COCOOCH}$ & & 0.8 & & & & & 1.2 & 2.0 \\
$\mathrm{CO}_{2}$ & 0.8 & 6.0 & 0.9 & 2.0 & 3.3 & 1.4 & 5.2 & 8.0 \\
$\mathrm{CO}_{3}$ & 0.2 & 0.3 & 1.4 & 3.3 & 5.4 & 1.1 & 0.4 & 0.3 \\
$\mathrm{H}_{2} \mathrm{O}$ & 0.4 & 4.5 & & & & 0.6 & 3.8 & 5.6 \\
\hline
\end{tabular}

Dedicated tests that measure composition of pyrolysis products are required before any model of detailed combustion chemistry can be implemented. Procedures to deduce the composition of volatiles can be based on extensive evolved gas analyses (EGA). A range of heat fluxes corresponding to typical stages of material thermal decomposition need be considered. Spontaneous ignition can be 
prevented, for example, by imposing appropriately designed metallic meshes on top of samples, which would take heat from the volatiles and reduce oxygen supply to the surface. We propose that both infrared spectroscopy and mass spectrometry to be employed to perform the EGA. The essential features of these techniques are briefly summarised below.

\subsubsection{Fourier Transform Infrared Spectroscopy (FTIR) and Gas Chromatography/Mass Spectrometry} (GC/MS)

Ideally, one should be able to quantify all volatile species from solid fuels by appropriately chosen method(s) that give reliable and reproducible results. Quantitative and real time analyses of the gaseous species have been attempted in the past by employing various types of hyphenated techniques such as Thermogravimetry or Simultaneous Thermal Analysis coupled either to Fourier Transform Infrared Spectroscopy (TGA/STA-FTIR) [39] or to Gas Chromatography/Mass Spectrometry (GC/MS) [40].

Figure 5. Indicative experimental set-up for evolved gas analysis.

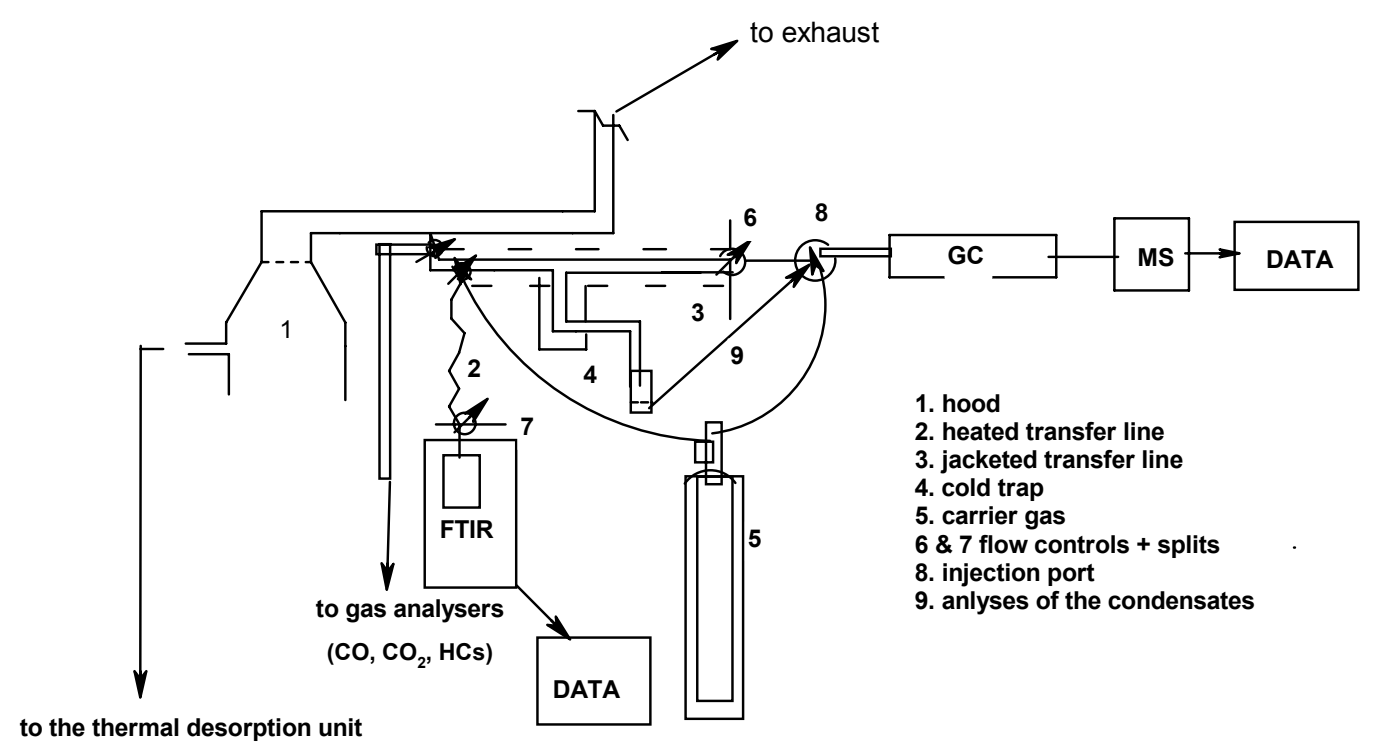

However, in spite of the vast amount of procedures cited in the literature and the enormous advances in instrumentation techniques and associated software, there are still a lot of issues that need be resolved before the best quantitative information can be deduced [41-43]. For example, the TGA/FTIR method of analysing the gaseous species often face problems such as low concentrations of species of interest and/or their inadequate IR response, condensation at the inter-phasing junctions, secondary undesirable chemical reactions along transfer lines that are generally kept at elevated temperatures, etc. Whilst mass spectrometry is highly sensitive for gaseous species analyses, for acquiring quantitative results it often needs to be pre-phased with some sort of gas chromatographic provision. This not only introduces a time delay into the analyses but also associated problems of off-line sampling and constraints on the availability of proper columns and/or the corresponding calibration standards. We propose to address the above issues, as best as possible, by proposing to inter-phase an FTIR and a GC/MS on-line to the exhaust hood of a cone calorimeter as shown in Figure 5 above. 
The temperature of volatiles at the point of tapping from the calorimeter exhaust line is estimated to be around $200{ }^{\circ} \mathrm{C}$ and at this stage they will be adequately diluted with a helium carrier gas stream. Thus the appropriately diluted volatiles will be transferred to the gas cell of the IR spectrometer, or to the injection loop of the GC, as the case may be, through heated/jacketed transfer lines (kept at $\sim 250{ }^{\circ} \mathrm{C}$ ). Any condensates of gases having a non-permanent nature and those having appreciable molecular weights will be trapped on to the "cold finger" and will be subjected to GC/MS analyses after being diluted with an appropriate solvent. It should be noted that the FTIR data obtained will be more of a "real time analysis" in nature, but incomplete owing to its failure to detect those components which are not sufficiently "infrared active". GC/MS analysis, on the other hand, owing to its superior sensitivity and universality in identifying all the components, has the disadvantage of not being a real time analytical technique due to the inherent time lag along the chromatographic column.

Assuming major volatile fragments are all appreciably infrared active, we propose to carry out quantitative measurements of these species by FT-IR, employing appropriate calibration standards. Comparative measurements will be performed using GC/MS to obtain more precise and reliable results. Here again, appropriate standardisation of the expected signals in the chromatogram will be performed using suitable calibrants before deducing quantitative information. Our proposal incorporates both types of techniques which essentially provide complementary data, leading to full picture in terms of quantitative evolved gas analyses. Furthermore, the proposed techniques are entirely novel in that no prior attempts have been made involving inter-phasing an infrared or a mass spectrometer directly with a cone calorimeter.

\subsection{Crucial Submodels and Implementation}

The present section describes key concepts that should lead to development of state-of-art model for combustion processes in Hybrid Rocket Engines. Particular submodels are discussed in detail in the following subsections, and also in the section IV.

\subsubsection{Flow Model}

A simplified configuration of a combustion chamber is presented in Figure 6 .

Figure 6. Configuration of a combustion chamber.

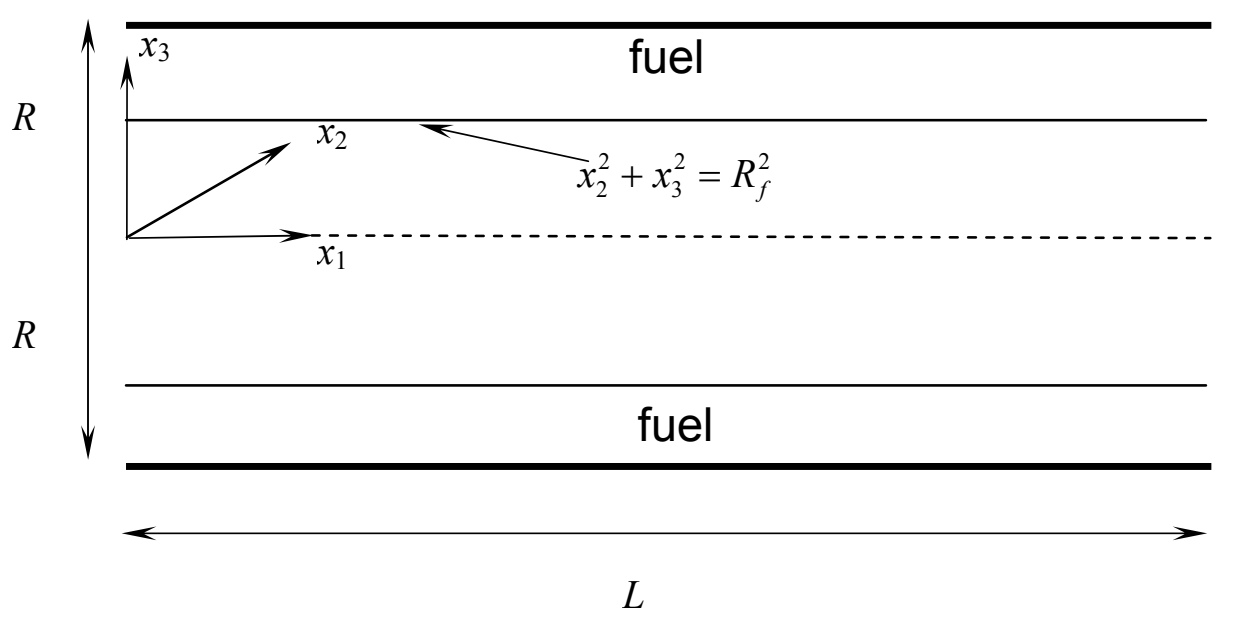


Assuming $\vec{x}=\left(x_{1}, x_{2}, x_{3}\right)$ is a space location, and $\vec{U}=\left(u_{1}, u_{2}, u_{3}\right)$ is a velocity vector, governing equations for the compressible flow may be written in the following form:

$$
\begin{gathered}
\frac{\partial \vec{Q}}{\partial t}+\frac{\partial \vec{E}_{i}}{\partial x_{i}}=\frac{\partial \vec{V}_{i}}{\partial x_{i}}+\vec{H} \\
\vec{Q}=\left(\begin{array}{l}
\rho \\
\rho u_{j} \\
\rho E \\
\rho \xi
\end{array}\right) ; \quad \vec{E}_{i}=\left(\begin{array}{l}
\rho u_{i} \\
\left(\rho u_{i} u_{j}+P \delta_{i j}\right) \\
(\rho E+P) u_{i} \\
\rho u_{i} \xi
\end{array}\right) ; \vec{V}_{i}=\left(\begin{array}{l}
0 \\
\sigma_{i j} \\
u_{j} \sigma_{i j}+\kappa(\nabla T)_{i} \\
\frac{\mu}{S c}(\nabla \xi)_{i}
\end{array}\right) ; \vec{H}=\left(\begin{array}{l}
0 \\
\rho(\vec{g}-\vec{a}) \\
S_{R} \\
0
\end{array}\right) \\
P=\frac{Y_{i}}{\mu_{i}} \rho R^{0} T
\end{gathered}
$$

Here $\vec{g}$ is gravity acceleration, $\vec{a}$ is rocket acceleration, $E=e_{i n}+\frac{u_{i} u_{i}}{2}$ is total energy per unit mass.

The specific internal energy $e_{i n}$ and the shear-stress tensor are given by:

$$
\begin{gathered}
e_{i n}=h-\frac{P}{\rho}=h_{i} Y_{i}-\frac{P}{\rho} ; \quad h_{i}=\Delta h_{f, i}^{0}+\int_{T^{0}}^{T} c_{p i} d T \\
\sigma_{i j}=\mu\left(\frac{\partial u_{i}}{\partial x_{j}}+\frac{\partial u_{j}}{\partial x_{i}}-\frac{2}{3} \delta_{i j} \frac{\partial u_{k}}{\partial x_{k}}\right)
\end{gathered}
$$

These flow dynamics equations are very standard, and there are several well known approaches that solve them with various degrees of approximation. They are supplemented by transport equation for the mixture fraction, $\xi$, which is used below for the analysis of diffusion combustion models. The detailed treatment of these equations in the framework of Implicit Large Eddy Simulations is discussed in Section 4, where specific example of computation is also presented.

\subsubsection{Combustion Modeling}

Combustion is treated as turbulent diffusion combustion, so that the approaches available for this particular mode of combustion may be applied.

Combustion model implementation requires knowledge of gas phase kinetics. Depending on success of extracting details of essential kinetic mechanisms (see discussion at the end of Section 3.3.2), approaches of different degree of accuracy may be exploited.

The simplest is a well-known fast chemistry model, which however may be quite a reasonable approximation in a view of high-temperature combustion process in pure oxygen environment. As most diffusion combustion models, it is based on mixture fraction concept, and is implemented in the following manner. Consider, as a simplest approximation, global single step combustion reaction (for example, for PMMA):

$$
\mathrm{C}_{5} \mathrm{H}_{8} \mathrm{O}_{2}+6 \mathrm{O}_{2} \rightarrow 5 \mathrm{CO}_{2}+4 \mathrm{H}_{2} \mathrm{O}
$$

or: 


$$
F+r \mathrm{O} \rightarrow(r+1) P ; \quad r=6
$$

where $F$ stands for fuel, $\mathrm{O}$ for oxygen, $P$ for product, and $r$ is fuel stoichiometric requirement.

The mixture fraction is defined as:

$$
\xi=\frac{\gamma-\gamma_{2}}{\gamma_{1}-\gamma_{2}}\left(\gamma=Y_{F}-\frac{Y_{O}}{r}\right)
$$

where "1" refers to the fuel stream; "2" refers to the oxidant stream.

Stoichiometric value of the mixture fraction is given by $\xi_{s t}=\frac{Y_{O}}{r Y_{F}+Y_{O}}$.

Note that mixture fraction is a so-called conserved scalar, i.e., it is not affected by chemical transformation, and correspondingly there is no source term in its transport equation.

\subsubsection{Fast Chemistry Approach}

Fast chemistry approach states, in essence, that fuel and oxygen cannot co-exist at a given spacetime location. They react immediately upon mixing, causing one of reactants (or both) to vanish. Distribution of reactant mass fractions in mixture fraction space, which corresponds to this physical description, is that consisting of broken profiles shown in Figure 7.

Figure 7. Reactant profiles in mixture fraction space under fast chemistry approach.

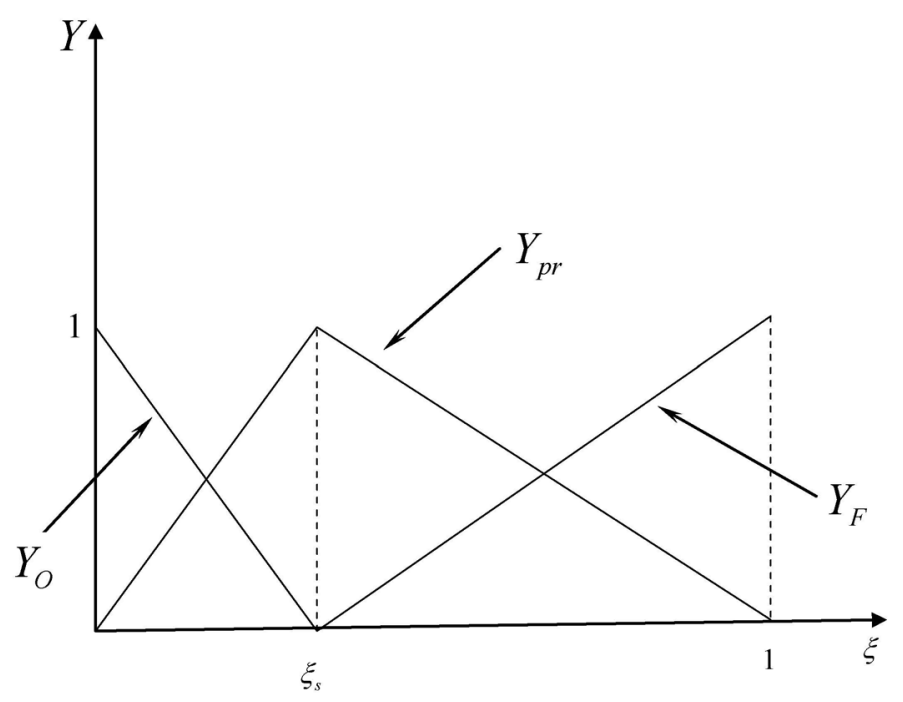

This is an example of "state relationships", i.e., properties dependence on mixture fraction in diffusion flame.

State relations $Y_{i}=Y_{i}(\xi)$ (Figure 7) can be described precisely as:

$$
\begin{array}{ll}
Y_{F}=0 ; Y_{O}=r \frac{\left(\xi_{s}-\xi\right)}{\left(1-\xi_{s}\right)} Y_{F}^{1} ; Y_{p r}=(r+1) \xi Y_{F}^{1} & 0 \leq \xi \leq \xi_{s} \\
Y_{F}=\frac{\left(\xi-\xi_{s}\right)}{\left(1-\xi_{s}\right)} Y_{F}^{1} ; Y_{O}=0 ; Y_{p r}=(r+1) \frac{\xi_{s}(1-\xi)}{\left(1-\xi_{s}\right)} Y_{F}^{1} & \xi_{s} \leq \xi \leq 1
\end{array}
$$

Individual mass fractions of the products are obtained as: 


$$
Y_{\mathrm{CO}_{2}}=\left[1+\frac{4}{5} \frac{\mu_{\mathrm{H}_{2} \mathrm{O}}}{\mu_{\mathrm{CO}_{2}}}\right]^{-1} \cdot Y_{p r} \quad Y_{\mathrm{H}_{2} \mathrm{O}}=\frac{4}{5} \frac{\mu_{\mathrm{H}_{2} \mathrm{O}}}{\mu_{\mathrm{CO}_{2}}}\left[1+\frac{4}{5} \frac{\mu_{\mathrm{H}_{2} \mathrm{O}}}{\mu_{\mathrm{CO}_{2}}}\right]^{-1} \cdot Y_{p r}
$$

With the help of mixture fraction combustion model, the set of Equations (6-8) is closed as follows. We have:

$$
\begin{gathered}
e_{i n}=E-\frac{u_{i} u_{i}}{2} \Rightarrow \rho e_{i n}=\rho E-\rho \frac{u_{i} u_{i}}{2} \Rightarrow \rho h-p=\rho E-\rho \frac{u_{i} u_{i}}{2} \Rightarrow \\
\Rightarrow p=\rho h-\rho E+\rho \frac{u_{i} u_{i}}{2} \Rightarrow \frac{Y_{i}}{\mu_{i}} R^{0} T=h-E+\frac{u_{i} u_{i}}{2} \Rightarrow \frac{Y_{i}(\xi)}{\mu_{i}} R^{0} T=h_{i}(T) Y_{i}(\xi)-E+\frac{u_{i} u_{i}}{2}
\end{gathered}
$$

Therefore:

$$
\frac{Y_{i}(\xi((\vec{x}, t)))}{\mu_{i}} R^{0} T=h_{i}(T) Y_{i}(\xi(\vec{x}, t))-E+\frac{u_{i} u_{i}}{2}
$$

becomes an additional constraint which closes the set of Equations (6-8).

Equations $(6-8,14)$ are written for instantaneous flow. In turbulent flow modelling one works with values averaged in certain sense. In the framework of Large Eddy Simulation (LES) approach, discussed in the present paper, the model must be closed in terms of resolved (filtered) quantities.

Resolved quantities $\bar{Y}_{i}(\vec{x}, t)$ should be obtained from instantaneous ones by involving sub-grid-scale probability density function (sgpdf):

$$
\bar{Y}_{i}(\vec{x}, t)=\int_{0}^{1} Y_{i}(\xi) P_{\xi}(\xi ; \vec{x}, t) d \xi
$$

Therefore, Equation (14) would be used in LES computations with $Y_{i}(\xi)$ replaced by $\bar{Y}_{i}(\vec{x}, t)$. In practical computations, the form of sgpdf is normally prescribed based on experimental insights. (Most comprehensive but extremely expensive Probability Density Function (PDF) approach solves transport equations for relevant pdfs). The most conventional form ( $\beta$-function) is set as:

$$
P_{\xi}(\xi ; \vec{x}, t)=\frac{\xi^{\alpha-1}(1-\xi)^{\beta-1}}{\int_{0}^{1} s^{\alpha-1}(1-s)^{\beta-1} d s}
$$

with:

$$
\alpha=\bar{\xi}\left[\frac{\bar{\xi}(1-\bar{\xi})}{\operatorname{var}(\xi)}-1\right] ; \beta=\frac{(1-\bar{\xi}) \alpha}{\bar{\xi}}
$$

Here $\bar{\xi}$ is resolved quantity of mixture fraction, and $\operatorname{var}(\xi)$ is sub-grid scale mixture fraction variance. The latter requires further modelling, which can be done, for example, following Branley and Jones [44] local equilibrium arguments:

$$
\operatorname{var}(\xi)=C_{\xi v} \Delta^{2} \frac{\partial \bar{\xi}}{\partial x_{k}} \frac{\partial \bar{\xi}}{\partial x_{k}} \quad \text { with } C_{\xi v} \approx 0.2
$$

( $\Delta$ can be taken as either filter width, or cubic root of cell volume). 


\subsubsection{Flamelet Approach}

The flamelet approach describes deviation from idealistic infinitely fast reaction, and therefore accounts for finite reaction rates. The flamelet formalism is well developed. The basic model of this approach is a Stationary Laminar Flamelet Model (SLFM). This has been extensively discussed in the literature (for example, by Peters [45]).

The SLFM equations have the form:

$$
\begin{aligned}
& \rho \frac{\chi_{s t}}{2} \frac{d^{2} Y_{i}}{d \xi^{2}}+\dot{\omega}_{i}=0 \\
& \rho \frac{\chi_{s t}}{2} \frac{d^{2} T}{d \xi^{2}}-\frac{h_{i}}{c_{p, i}} \dot{\omega}_{i}=0
\end{aligned}
$$

and extend simple fast chemistry state relationships to the case of finite reaction rates.

Here $\chi=2 D \frac{\partial \xi}{\partial x_{k}} \cdot \frac{\partial \xi}{\partial x_{k}}$ is the scalar dissipation rate, and $\chi_{s t}$ is its value at stoichiometry. (Conventional summation over repeating indices is assumed in (19), (20), and further on).

To be useful, these equations require fairly detailed chemical kinetics scheme. Here $\dot{\omega}_{i}$ is the rate of production of the $i$-th species (positive or negative depending whether species is produced or consumed) (NOTE: another possible form of the model assumes $\chi=\chi_{s t} \cdot f(\xi)$, rather than $\chi=\chi_{s t}=$ const in the above equations. Particular form of $f(\xi)$ can be found in the literature, e.g., $[45-48]$.

Upon solution of the SLFM equations, two-parameter library of species profiles in a laminar flame:

$$
Y_{i}=Y_{i}\left(\xi, \chi_{s t}\right)
$$

can be established.

Resolved quantities in a turbulent flow are recovered as follows:

$$
\bar{Y}_{i}(\vec{x}, t)=\int_{0}^{\infty} \int_{0}^{1} Y\left(\xi, \chi_{s t}\right) P_{\xi \chi}\left(\xi, \chi_{s t} ; \vec{x}, t\right) d \xi d \chi_{s t}
$$

where the joint sgpdf $P_{\xi \chi}\left(\xi, \chi_{s t} ; \vec{x}, t\right)$ of $\xi$ and $\chi_{s t}$ variables must be known.

The choice of this joint sgpdf is much more challenging, compared to the fast chemistry case, due to lack of experimental data. Statistical independence $P_{\xi \chi}\left(\xi, \chi_{s t} ; \vec{x}, t\right)=P_{\xi}(\xi ; \vec{x}, t) \cdot P_{\chi}\left(\chi_{s t} ; \vec{x}, t\right)$ may be assumed as a reasonable first step. In this form, one of possible choices is:

$$
P_{\xi}(\xi ; \vec{x}, t)=\frac{\xi^{\alpha-1}(1-\xi)^{\beta-1}}{\int_{0}^{1} s^{\alpha-1}(1-s)^{\beta-1} d s}
$$

(i.e., the same $\beta$-function as above), and:

$$
P_{\chi}\left(\chi_{s t} ; \vec{x}, t\right)=\frac{1}{\sigma \sqrt{2 \pi} \chi_{s t}} \exp \left(-\frac{1}{2 \sigma^{2}}\left(\ln \chi_{s t}-\varsigma\right)^{2}\right)
$$


where $\bar{\chi}=\exp \left(\varsigma+\frac{1}{2} \sigma^{2}\right)$ is resolved value calculated from the $\bar{\xi}$ field:

$$
\bar{\chi}=2 D \frac{\partial \bar{\xi}}{\partial x_{k}} \cdot \frac{\partial \bar{\xi}}{\partial x_{k}}=2 \frac{\mu}{\rho S c} \frac{\partial \bar{\xi}}{\partial x_{k}} \cdot \frac{\partial \bar{\xi}}{\partial x_{k}}
$$

and:

$$
\sigma^{2} \approx 2([45,49])
$$

Another possibility is given by:

$$
\mathrm{P}_{\xi}(\xi ; \vec{x}, t)=\frac{\xi^{\alpha-1}(1-\xi)^{\beta-1}}{\int_{0}^{1} \xi^{\alpha-1}(1-\xi)^{\beta-1} \mathrm{~d} \xi}-\frac{\Gamma(\alpha+\beta)}{\Gamma(a) \Gamma(b)} \xi^{\alpha-1}(1-\xi)^{\beta-1}
$$

where $\Gamma(x)$ is the gamma function $\Gamma(x)=\int_{0}^{\infty} e^{-t} t^{x-1} d t$.

The parameters $\alpha$ and $\beta$ here are the functions of the mean value and the variance of the mixture fraction:

$$
\alpha=\frac{\bar{\xi}^{2}(1-\bar{\xi})}{\operatorname{var}(\xi)}-\bar{\xi}, \beta=\frac{\bar{\xi}(1-\bar{\xi})^{2}}{\operatorname{var}(\xi)}-(1-\bar{\xi})-\alpha\left(\frac{1-\bar{\xi}}{\bar{\xi}}\right)
$$

A model for the variance of $\xi$ can be found in Branley and Jones [44]. There is lack of data to construct scalar dissipation part of sgpdf. Some indicative mixing configurations may be used as a first step, however. One can consider, fro example, shear layer flow study of Pantano et al. [50] (Figure 8). This provides pdf data for the scalar dissipation rate.

Figure 8. Schematic diagram of the temporally evolving shear layer with counter-directed streams $u_{1}=-\frac{1}{2} \Delta u, \rho_{1}=\rho_{0}, Y_{O}=Y_{O, 0} ; u_{2}=-\frac{1}{2} \Delta u, \rho_{2}=\rho_{0}, Y_{F}=Y_{F, 0} \quad(\Delta u$ is prescribed velocity difference).

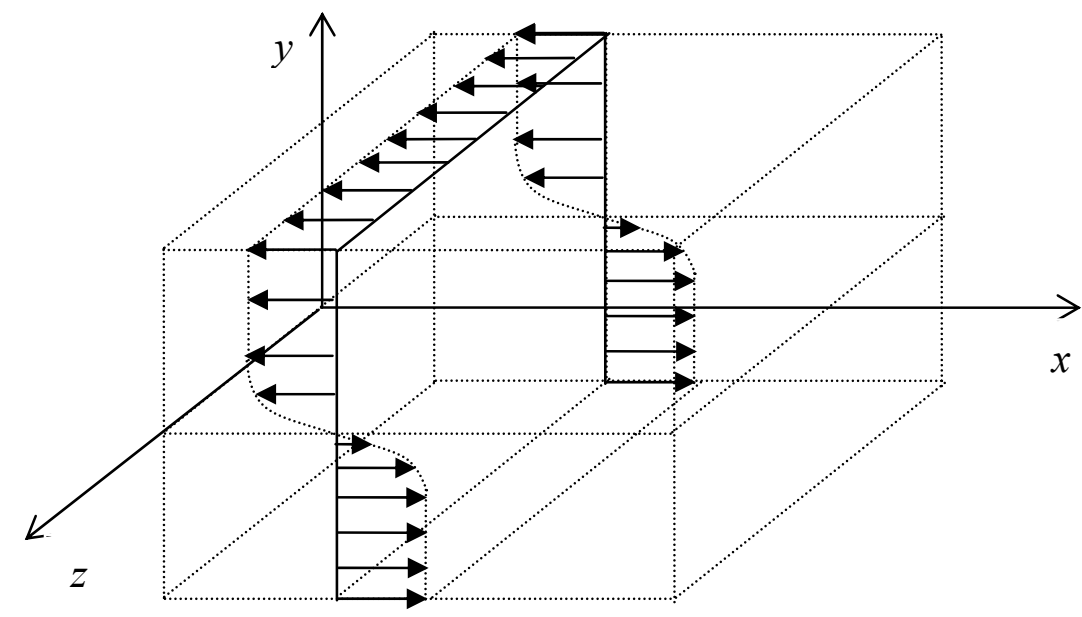

Pdf for the scalar dissipation rate can be taken as (assuming below $x=x_{1}, y=x_{2}$ and $z=x_{3}$ ): 


$$
P\left(\ln \left(\chi \delta_{\omega} / \Delta u\right)\right)=\frac{1}{\sqrt{2 \pi \sigma_{x}^{2}}} \exp \left(-\frac{\left(\ln \left(\chi \delta_{\omega} / \Delta u\right)-\mu_{x}\right)^{2}}{2 \sigma_{x}^{2}}\right)
$$

where $\Delta u$ is the velocity difference of the two streams, and $\delta_{\omega}$ is the initial vorticity thickness of the shear layer, defined as:

$$
\delta_{\omega}=\frac{\Delta u}{\left(\partial \widetilde{u}_{1} / \partial x_{2}\right)_{\max }}
$$

Here $\widetilde{u}_{1}$ denotes the Favre-averaged velocity in the $x_{1}$ direction. The values of the mean $\mu_{x}$ and the standard deviation $\sigma_{x}$ can be found in Pantano et al. [50]. Results of flamelet modelling should be compared to experiments. It is expected that SLFM should provide fair results in the view of fast kinetics of major heat-generating reactions. If, however, this approach is found to be deficient, extension to unsteady flamelet model is possible, although its implementation in LES is not as straightforward [51].

\subsubsection{Fuel Regression Model}

Gas flow must be coupled to the appropriate model for solid fuel thermal behaviour and decomposition. Such a model can be developed along the following lines. Without the loss of generality, we can consider at the moment homogeneous fuel with invariable material properties.

Let $T_{f}=T_{f}(\vec{x}, t)$ be solid fuel temperature. At each cross-section $x_{1}=$ const fuel surface $S_{f}$ is described by the time-dependent function $F_{x_{1}, t}\left(x_{2}, x_{3}\right)$ so that $S_{f}=\left\{\vec{x}, t: F_{x_{1}, t}\left(x_{2}, x_{3}\right)=0\right\}$.

Initially (Figure 6):

$$
F_{x_{1}, 0}\left(x_{2}, x_{3}\right)=x_{2}^{2}+x_{3}^{2}-R_{f}^{2}
$$

Three-dimensional heat transfer equation:

$$
\frac{\partial T_{f}}{\partial t}=\eta \cdot \Delta T \quad \vec{x}, t: F_{x_{1}, t}\left(x_{2}, x_{3}\right)>0
$$

can be solved with $\eta$ being thermal diffusivity of the fuel. At the gas-solid interface, boundary condition (heat balance) can be written as follows:

$$
\begin{aligned}
& -\left.\kappa \frac{\partial T}{\partial \vec{n}}\right|_{g}+\dot{q}_{R}^{\prime \prime}=-\left.\kappa_{s} \frac{\partial T}{\partial \vec{n}}\right|_{s}-\rho_{s}\left\|\frac{d\left(S_{f} \cap\{\alpha \cdot \vec{n}\}\right)}{d t}\right\| \Delta H_{s g} \vec{x} \in S_{f} \\
& \vec{n}=\frac{\nabla F_{x_{1}, t}}{\left|\nabla F_{x_{1}, t}\right|} ; \quad \nabla F_{x_{1}, t}\left(x_{2}, x_{3}\right)=\left(\frac{\partial F_{x_{1}, t}}{\partial x_{2}}, \frac{\partial F_{x_{1}, t}}{\partial x_{3}}\right)
\end{aligned}
$$

where " $s$ " refer to solid (fuel) side, " $g$ " refer to gas side, $\dot{q}_{R}^{\prime \prime}$ is incident radiative flux, $\Delta H_{s g}$ is heat of pyrolysis (positive if thermal decomposition is exothermal, and negative if decomposition is endothermal). Here $\left(S_{f} \cap\{\alpha \cdot \vec{n}\}\right)$ denotes a point of intersection between fuel surface and the line $\{\alpha \cdot \vec{n}\} \alpha \in \mathfrak{R}$, normal to the surface (Figure 9).

Further, $\left\|\frac{d\left(S_{f} \cap\{\alpha \cdot \vec{n}\}\right)}{d t}\right\|$ denotes the rate of the fuel surface regression in the $\vec{n}$-direction. 
Figure 9. Local boundary $\left(x_{1}=\right.$ const $)$ of the fuel surface.

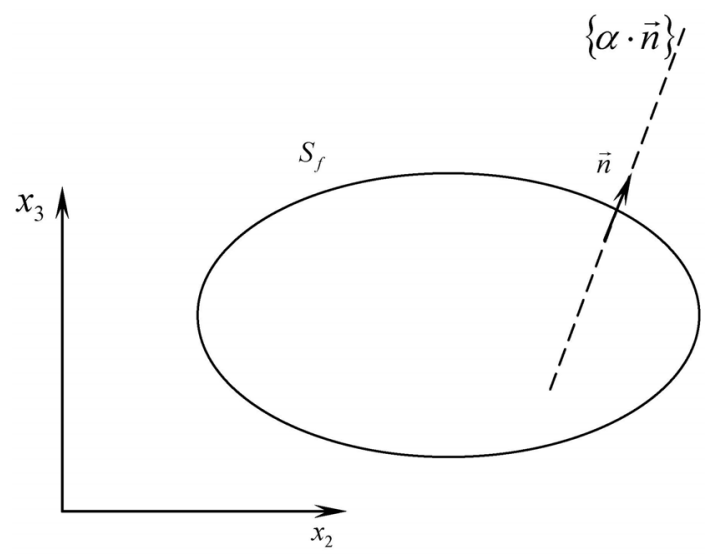

The auxiliary condition that must be imposed is:

$$
\left\|\frac{d\left(S_{f} \cap\{\alpha \cdot \vec{n}\}\right)}{d t}\right\|=\frac{\dot{m}_{p}}{\rho_{f}}
$$

where $\rho_{f}$ is fuel density, $\dot{m}_{p}$ is fuel thermal decomposition rate. The approaches to model the rate $\dot{m}_{p}$ have been discussed above (sub-Section 3.2). Evolution equation for the surface-defining function $F_{x_{1}, t}\left(x_{2}, x_{3}\right)$ is:

$$
\frac{\partial F_{x_{1}, t}\left(x_{2}, x_{3}\right)}{\partial t}=-\left(\nabla F_{x_{1}, t}\left(x_{2}, x_{3}\right) \cdot \frac{d\left(\widehat{S_{f} \cap\{\alpha \cdot \vec{n}\}}\right)}{d t}\right)
$$

where $(\cdot)$ on RHS denotes the scalar product, with the initial condition:

$$
F_{x_{1}, 0}\left(x_{2}, x_{3}\right)=x_{2}^{2}+x_{3}^{2}-R_{f}^{2}
$$

Other boundary conditions at the fuel surface are as follows:

$$
\begin{aligned}
& \vec{U}=0 \quad \vec{x} \in S_{f} \\
& \xi=1 \quad \vec{x} \in S_{f} \\
& T_{f}^{s}=T_{g}^{s} \quad T_{f}^{s} \text {-fuel surface temperature } \vec{x} \in S_{f}
\end{aligned}
$$

The last condition (39) implies continuity of the temperature profile with $T_{f}^{s}$ being fuel surface temperature, and $T_{g}^{s}$ being a limiting (upon approaching the surface) gas temperature. Previous results are normally derived for low (e.g., flame-induced velocities). In a rapid flow, rate of monomer vaporisation may be increased by mechanical disruption of polymer layer. Such enhancement can be approximately accounted for by the modification:

$$
\tilde{\dot{m}}_{p}=\dot{m}_{p}\left(1+f\left(c_{f}\right)\right)
$$

where $\dot{m}_{p}=\rho_{f}\left\|\frac{d\left(S_{f} \cap\{\alpha \cdot \vec{n}\}\right)}{d t}\right\|$ is a "regular" fuel decomposition rate as above, and $f$ is a function of local friction coefficient $c_{f}$ and material properties. Experiments are needed to approximate the form of the function $f$. 


\subsubsection{Radiation Heat Transfer}

The account of radiation heat exchange is very important in general in combustion systems. In Hybrid Engines fuel regression rate is under-predicted if radiative flux to the surface is not taken into account. It is clear that degree of under-prediction will depend strongly on the nature of the fuel, which in Hybrid Engines may be important in a view of experimental observations. Both experimental [52] and numerical [53] observations suggest that radiation may account for approximately from $10 \%$ to $45 \%$ of total heat flux to the surface, and as expected radiation contribution grows with the scale of engine. Therefore, for full scale engines of most practical interest, radiation heat transfer must be taken into account. Current radiation modelling efforts [53] calibrate the radiation model against experimental data to achieve reasonable results. As this is not quite satisfactory, more substantial development of radiation models is required. There are several approaches that may be taken. They are discussed below.

\subsubsection{Radiation Correction}

For practical applications it may be quite sufficient to implement simple radiation correction. This can be done, for example, through modification of the flamelet model. To include extra an effect, such as radiation heat loss, additional parameter is required in the flamelet parameterization. One may introduce an enthalpy defect:

$$
\Delta h=h-\left[h_{O}+\xi\left(h_{F}-h_{O}\right)\right]
$$

so that the state relations become:

$$
Y_{i}=Y_{i}(\xi, \chi, \Delta h)
$$

Details of this approach (Figure 10) are given by Hossain et al. [54].

Figure 10. Modification of flamelet library for radiation loss.

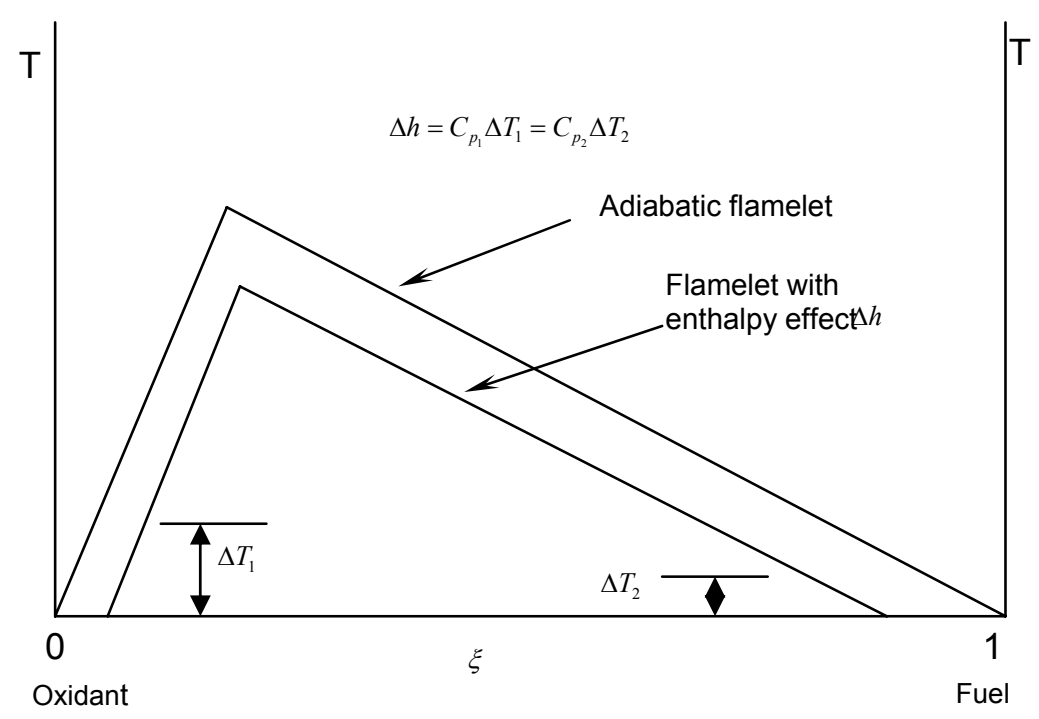

Heat sink $S_{R}$ is added to the energy Equation (7).

Source term may be evaluated in the optically thin limit as: 


$$
S_{R}=4 \sigma a_{m}(\vec{x})[T(\vec{x})]^{4}
$$

where $a_{m}$ is local absorption coefficient.

The absorption coefficient can be approximated as $a_{m} \approx 0.28 \exp (-T / 1135)$ [55].

Incident radiative flux on the fuel surface is calculated in this approximation as:

$$
\left.\dot{q}_{R}^{\prime \prime}\right|_{\vec{x} \in S_{f}}=4 \sigma \int_{V} \frac{\sin (\beta)}{l^{2}} a_{m}(\vec{x})[T(\vec{x})]^{4} d v
$$

where $V$ is volume of gas computational region; $\beta$ is angle required to define projected area of the surface element (Figure 11), perpendicular to the ray between the surface element and the gas elementary volume $d v ; l$ is distance between surface and volume elements.

Figure 11. Calculation of incident radiative flux.

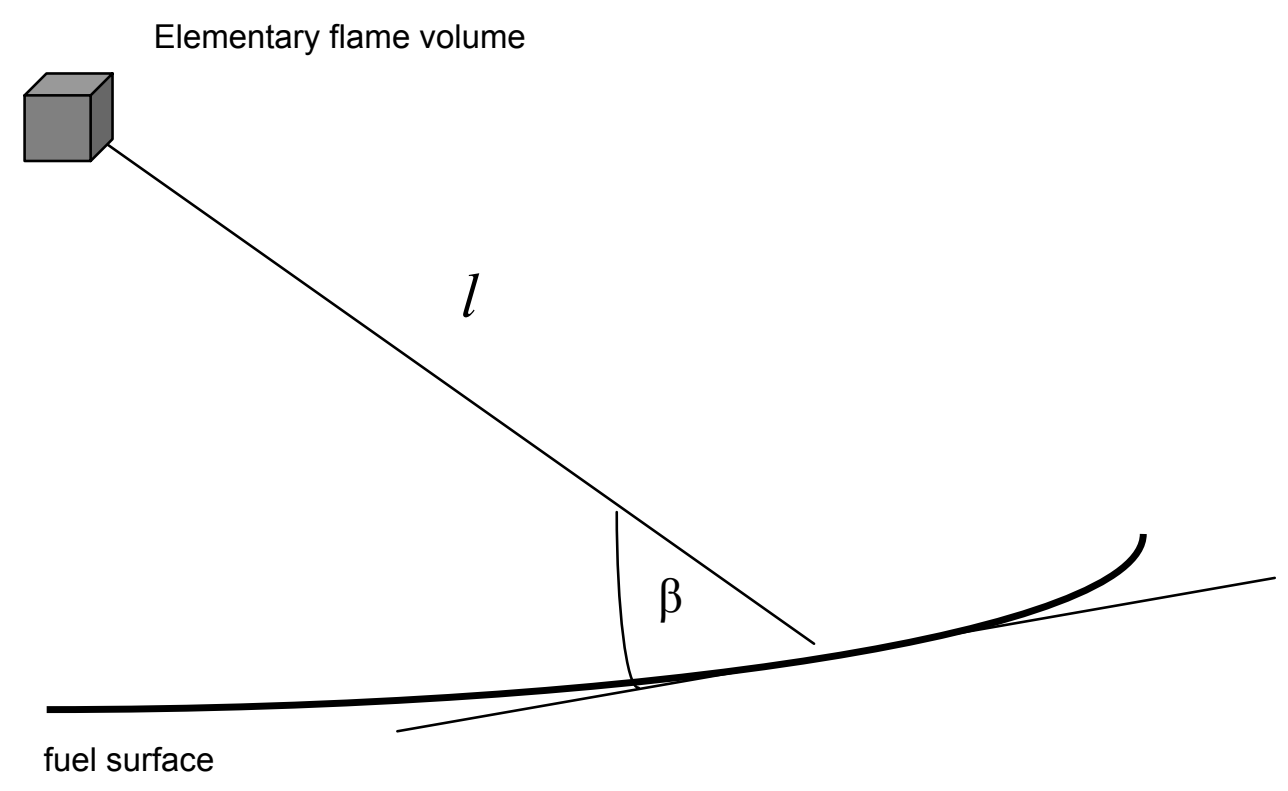

\subsubsection{Comprehensive Radiation Modeling}

Comprehensive radiation treatment (an approximate solution for the Radiative Transfer Equation, RTE) may be implemented using various available methods methods. One potential possibility is the use of Discrete Transfer Radiation Method (DTRM) of Lockwood and Shah [56]. This method has been applied widely for combustion system studies. Brief details of this method are as follows $[55,56]$.

In a grey gas approximation, the transport of total (wavelength-integrated) radiation intensity $I=\int_{0}^{\infty} I_{\lambda}(\Omega) d \lambda$ over distance $s$ along the discrete ray is described by:

$$
\frac{d I}{d s}=-\left(\alpha_{g}+\alpha_{s}\right) I(s)+\frac{\sigma}{\pi}\left(\alpha_{g}+\alpha_{s}\right) T^{4}
$$

This equation can be conveniently solved in discretized form applying consecutively:

$$
I_{n+1}=I_{n} \exp (-\alpha s)+\frac{\sigma T^{4}}{\pi}(1-\exp (-\alpha s))
$$


for intensities of the ray as it enters $\left(I_{n}\right)$ and leaves $\left(I_{n+1}\right)$ control volumes.

Overall absorption coefficient for the soot and gas mixture can be approximated (Smith's model) as:

$$
\begin{aligned}
& \alpha=\alpha_{g}+\alpha_{s}-\alpha_{g} \alpha_{s} \\
& \alpha_{g} \approx 0.28 \exp (-T / 1135) ; \quad \alpha_{s} \approx 1264 f_{v} T
\end{aligned}
$$

where $f_{v}$ is the soot volume fraction. Examples of application of this model to combustion are given in Novozhilov [57,58].

\subsubsection{Injector Spray Model}

This subsection provides brief description of a particular (Eulerian-Lagrangian) method, which can be applied to modelling liquid oxygen $\left(\mathrm{LO}_{\mathrm{x}}\right)$ injector flow. It is assumed that liquid oxygen stream is atomized into droplets that evaporate further downstream and thus provide gaseous oxidant supply for diffusion combustion.

The Eulerian-Lagrangian models for two-phase flow have been studies intensively (see, for example Kuo [59], Gosman and Ioannides [60], Faeth [61], Crowe et al. [62,63]. Key steps in application of this technique to modelling of liquid sprays are as follows.

Particles (droplets) are treated in a Lagrangian manner. The equation of a single spherical droplet motion in a turbulent flow field forms the base of such a model. In a sufficiently general form, this equation is $([59,61])$ :

$$
\begin{aligned}
& \frac{\pi}{6} d_{p}^{3} \rho_{p} \frac{d \vec{U}_{p}}{d t}=\frac{\pi}{8} d_{p}^{2} \rho C_{D}\left|\vec{U}-\vec{U}_{p}\right|\left(\vec{U}-\vec{U}_{p}\right) \\
& +\frac{\pi}{6} d_{p}^{3} \rho \frac{D \vec{U}}{D t}+\frac{\pi}{12} d_{p}^{3} \rho C_{I} \frac{d}{d t}\left(\vec{U}-\vec{U}_{p}\right) \\
& +\frac{3}{2} d_{p}^{2}(\pi \rho \mu)^{1 / 2} C_{B} \int_{0}^{t} \frac{(d / d \xi)\left(\vec{U}-\vec{U}_{p}\right)}{(t-\xi)^{1 / 2}} d \xi+\vec{F}_{e}
\end{aligned}
$$

The terms on the right hand side represent, respectively, drag; the force due to static pressure gradient; the force on sphere due to inertia of adjacent fluid being displaced by its motion (virtual-mass term); Basset force to account for effects of deviation of the flow from a steady flow pattern around the sphere, and external body-force, e.g., gravity.

The effect of lift forces, which only appear when there are significant velocity gradients normal to the trajectory of the particle, as well as Magnus effect, are ignored here [61]. Detailed consideration of relative importance of different terms is presented by Faeth [61].

Experience shows that in most practical applications pressure gradient, virtual mass, and Basset force terms may be neglected $([61,64,65])$. Therefore, the following simplified equation of droplet motion can be adopted with a reasonable accuracy:

$$
\frac{d \vec{U}}{d t}=\left(\frac{3 \rho C_{D}}{4 d_{p} \rho_{p}}\right)\left(\vec{U}-\vec{U}_{p}\right)\left|\vec{U}-\vec{U}_{p}\right|+\vec{g}
$$


Equation (50) is non-linear, and in general case has to be solved by numerical integration. (Some particular cases where analytical integration is possible are considered by Novozhilov [66,67].)

Drag coefficient appearing in the Equation (50) should be specified from experimental correlations. Such correlations for the spherical particle have been developed extensively. One can use, for example, approximation by Putnam [68]:

$$
C_{D}= \begin{cases}24 / \operatorname{Re}_{p}\left(1+\operatorname{Re}_{p}^{2 / 3} / 6\right) & \operatorname{Re}_{p}<1000 \\ 0.44 & \operatorname{Re}_{p}>1000\end{cases}
$$

where the particle Reynolds number $\operatorname{Re}_{p}=\frac{d_{p}\left|\vec{U}_{p}-\vec{U}\right| \rho}{\mu}$ is based on droplet diameter.

There are similar correlations, discussed in the literature [69], that provide effectively same accuracy as (51), for example:

$$
C_{D}=\left\{\begin{array}{lc}
27 / \operatorname{Re}_{p}^{0.84} & \operatorname{Re}_{p}<80 \\
0.271 \operatorname{Re}_{p}^{0.217} & 80<\operatorname{Re}_{p}<10^{4}
\end{array}\right.
$$

In flows of practical interest droplets are also subjected to turbulent dispersion. To model this effect, some kind of stochastic particle dispersion model is required. The simplest way, in the framework of the $k-\varepsilon$ approach has been proposed by Gosman and Ioannides [60].

Dispersion calculation requires instantaneous gas velocity as an input into Equation (50). From the $k-\varepsilon$ point of view, the instantaneous gas velocity within the eddy is obtained using the computed value of the turbulence kinetic energy, $k$. Assuming isotropic turbulence and Gaussian distribution for fluctuating components (with the standard deviation $(2 k / 3)^{1 / 2}$ ), this distribution can be sampled randomly to obtain the instantaneous velocity $U^{\prime}$.

As a droplet travels through the flow, it interacts with individual eddies. Each eddy along the droplet path deflects it according to the eddy's instantaneous velocity. Particle trajectory may be determined in a random walk manner, using estimations for the eddy lifetime and droplet transit time through the eddy. The eddy lifetime can be estimated ([60]) as:

$$
t_{e}=\frac{L_{e}}{\left|\vec{U}^{\prime}\right|}
$$

where the characteristic size of the eddy is assumed to be equal to dissipation length scale:

$$
L_{e}=\frac{C_{\mu}^{3 / 4} k^{3 / 2}}{\varepsilon}
$$

Shuen et al. [64] found better agreement with measurements employing:

$$
t_{e}=\frac{L_{e}}{\left(\frac{2}{3} k\right)^{\frac{1}{2}}}
$$

Droplet transit time may be found from the linearised equation of the particle motion in a uniform flow: 


$$
t_{p}=-\tau_{p} \ln \left(1-\frac{L_{e}}{\tau_{p}\left|\vec{U}-\vec{U}_{p}\right|}\right)
$$

where $\tau_{p}$ is the particle relaxation time:

$$
\tau_{p}=\frac{4 \rho_{p} d_{p}}{3 \rho C_{D}\left|\vec{U}-\vec{U}_{p}\right|}
$$

Equation (57) has no solution for $L_{e}>\tau_{p}\left|\vec{U}-\vec{U}_{p}\right|$. This may be interpreted as particle capture by the eddy. The interaction time in this case becomes equal $t_{e}$. Time of interaction between the particle and the eddy is taken as the shortest of the two times, $t_{e}$ and $t_{p}$.

Other ways of modeling particle dispersion, such as gradient diffusion models, are not particularly convenient for incorporation into the Lagrangian framework. They are discussed by Faeth [61]. Different modifications of dispersion models suitable for Lagrangian formulation are presented in Shirolkar et al. [70].

Along with the momentum Equation (50), heat and mass transfer equations for the droplet need be considered. Assuming uniform temperature for the droplet (i.e., small Biot number), these equations take the form:

$$
\frac{d\left(m_{p} c_{p, p} T_{p}\right)}{d t}=\dot{q}_{p}+L_{f g} \frac{d m_{p}}{d t}
$$

and:

$$
\frac{d m_{p}}{d t}=-\operatorname{Sh} \pi d_{p} \frac{\kappa_{g}}{c_{p g}} \ln \left(1+B_{m}\right)
$$

where the Spalding's number $B_{m}$ is defined as:

$$
B_{m}=\frac{Y_{l, s}-Y_{l, \infty}}{1-Y_{l, s}}
$$

Partial pressure of vapor at the droplet surface is assumed to correspond to the saturation conditions at a given temperature.

Nusselt and Sherwood numbers are usually calculated through the Ranz-Marshall correlations. These are given as functions of Reynolds, Prandtl and Schmidt numbers [71,72]:

$$
N u=2.0+0.6 \operatorname{Re}_{p}^{1 / 2} \operatorname{Pr}^{1 / 3} ; S h=2.0+0.6 \operatorname{Re}_{p}^{1 / 2} S c^{1 / 3}
$$

Alternatively, the formulae by Faeth and Lazar [73] may be used to make corrections for the moving droplet:

$$
\begin{aligned}
& \frac{\dot{m}_{p}}{\left(\dot{m}_{p}\right)_{\mathrm{Re}_{p}=0}}=1+\frac{0.278 \operatorname{Re}_{p}^{1 / 2} \mathrm{Sc}^{1 / 3}}{1+1.232 /\left[\operatorname{Re}_{p} \mathrm{Sc}^{4 / 3}\right]^{1 / 2}} \\
& \frac{h_{c}}{\left(h_{c}\right)_{\mathrm{Re}_{p}=0}}=1+\frac{0.278 \operatorname{Re}_{p}^{1 / 2} \operatorname{Pr}^{1 / 3}}{1+1.232 /\left[\operatorname{Re}_{p} \operatorname{Pr}^{4 / 3}\right]^{1 / 2}}
\end{aligned}
$$


Here the heat and mass transfer coefficients for the quiescent droplet are:

$$
\begin{aligned}
& \frac{\left(h_{c}\right)_{\mathrm{Re}_{p}=0} d_{p}}{k}=\frac{\dot{m} c_{p, p} / \pi d_{p} \kappa_{g}}{\exp \left(\dot{m} c_{p, p} / 2 \pi d_{p} \kappa_{g}\right)-1} \\
& (\dot{m})_{\operatorname{Re}_{p}=0}=2 \pi d_{p} \rho D \ln \left(\frac{Y_{l, \infty}-1}{Y_{l, s}-1}\right)
\end{aligned}
$$

These expressions provide reasonable correlation of the existing data for $\operatorname{Re}<2 \times 10^{3}$ [61].

Solutions for the gas and dispersed (liquid) phases need be coupled. This may be achieved, for example, by the use of the Particle-Source-In-Cell (PSI-CELL) model [62]. This model is based on the earlier idea of Migdal and Agosta [74] that particles can be considered as sources of mass, momentum and energy to continuum phase. The method solves equations for each phase interchangeably, and update particle source terms until a converged solution is obtained. Full details of this technique can be found in $[62,63,70]$.

The specification of initial droplet size and velocity distributions is crucial for the accurate prediction of spray/flow interaction. Heat, mass and momentum transfer rates are sensitive to these parameters [57,58]. Common practice [57,58] is to specify initial conditions for a number of trajectories which differ by initial droplet diameter, position and velocity. These discrete distributions can be chosen to match available characteristics of a particular spray. In the view of large number of droplets, each trajectory is supposed to represent a group of droplets with the same initial conditions.

There are alternative approaches ([75]) where spray is represented by a number of "superdrops". With the LES treatment of flow dynamics, particle Lagrangian equations need be advanced in parallel with the fluid phase equations. Interactions of particles with large, unsteady vortices are computed explicitly. Turbulent dispersion is handled automatically at the resolved scales, but specific dispersion model may be needed for subgrid-scale effects.

At present stage, one-way momentum coupling can be realistically implemented in LES, so that droplet trajectories will be affected by momentum transfer from the fluid (and external forces), but not vice versa.

Effect of sub-grid turbulence on drag force should be taken into account, which can be done recalculating the particle Reynolds number with the total viscosity $\left(\mu+\mu_{S G S}\right)$. Heat-up and evaporation rates can be calculated by conventional procedures described above.

Liquid oxygen $\left(\mathrm{LO}_{\mathrm{x}}\right)$ properties of injected droplets can be specified from table data (liquid oxygen has a density of approximately $1.14 \mathrm{~g} / \mathrm{cm}^{3}$ and boiling point of $90.188 \mathrm{~K}\left(-182.96^{\circ} \mathrm{C}\right)$ at $\left.101.325 \mathrm{kPa}\right)$.

In the described model framework it is assumed that there is a well defined vaporisation front so that mixing and combustion occur downstream from that front.

If the flame envelope is developing around droplets, i.e., spray combustion needs be considered, then a different approach is required. Such a combustion regime can be dealt with by appropriate spray combustion models. Example of such modelling in application to Hybrid Rocket Engines is given by Lin and Chiu [76]. 


\section{Preliminary Simulations}

Preliminary results of boundary layer combustion modelling using Implicit Large Eddy Simulations are presented in this section. These results demonstrate essential features of flame development in Hybrid Engine chamber, and will be developed to much more comprehensive model in the future.

Governing equations are the compressible Navier-Stokes equations which are the conservation of total mass, momentum and total energy:

$$
\begin{aligned}
& \frac{\partial \rho}{\partial t}+\frac{\partial \rho u_{j}}{\partial x_{j}}=0 \\
& \frac{\partial \rho u_{i}}{\partial t}+\frac{\partial\left(\rho u_{i} u_{j}+\delta_{i j} p\right)}{\partial x_{j}}=\frac{\partial \sigma_{i j}}{\partial x_{j}} \\
& \frac{\partial e}{\partial t}+\frac{\partial(e+p) u_{j}}{\partial x_{j}}=\frac{\partial \sigma_{i j} u_{i}}{\partial x_{j}}-\frac{\partial q_{j}}{\partial x_{j}}
\end{aligned}
$$

where $\rho\left[\mathrm{kg} / \mathrm{m}^{3}\right]$ denotes the density, $u_{i}(i=1,2,3)[\mathrm{m} / \mathrm{s}]$ are the Cartesian velocity components corresponding to $(u, v, w), p[\mathrm{~Pa}]$ the pressure, $e\left[\mathrm{~J} / \mathrm{m}^{3}\right]$ the total energy per unit volume, respectively. In order to close the above equations, the equation of state for perfect gas is employed which is defined as:

$$
p=(\gamma-1)\left\{e-\frac{\rho}{2}\left(u^{2}+v^{2}+w^{2}\right)\right\}
$$

where $\gamma$ denotes the ratio of specific heats. According to the Stoke's hypothesis which assumes that the bulk viscosity can be neglected, the shear-stress tensor for a Newtonian fluid is given by:

$$
\sigma_{i j}=2 \mu\left(S_{i j}-\frac{\delta_{i j}}{3} S_{k k}\right)
$$

where $\mu$ denotes the coefficient of molecular viscosity, $S_{i j}$ the components of rate-of-strain tensor which are defined as follows:

$$
S_{i j}=\frac{1}{2}\left(\frac{\partial u_{i}}{\partial x_{j}}+\frac{\partial u_{j}}{\partial x_{i}}\right)
$$

The heat flux $q_{j}\left[\mathrm{~J} /\left(\mathrm{m}^{2} \mathrm{~s}\right)\right]$ is given by:

$$
q_{j}=-\kappa \frac{\partial T}{\partial x_{j}}
$$

where $T[\mathrm{~K}]$ denotes the temperature and $\kappa[\mathrm{J} /(\mathrm{m} \mathrm{s} \mathrm{K})]$ the thermal conductivity defined by:

$$
\kappa=\frac{\mu c_{p}}{\operatorname{Pr}}
$$

where $c_{p}[\mathrm{~J} /(\mathrm{kg} \mathrm{K})]$ denotes the specific heat at constant pressure and $\operatorname{Pr}$ the Pandtl number, respectively. 
When the finite volume method is applied to Equations (64-66), the integral formulation of governing equations is obtained as follows:

$$
\begin{aligned}
& \frac{\partial}{\partial t} \int_{\Omega} \rho d V+\oint_{\partial \Omega} \rho u_{j} n_{j} d S=0 \\
& \frac{\partial}{\partial t} \int_{\Omega} \rho u_{i} d V+\oint_{\partial \Omega}\left(\rho u_{i} u_{j}+\delta_{i j} p\right) n_{j} d S=\oint_{\partial \Omega} \sigma_{i j} n_{j} d S \\
& \frac{\partial}{\partial t} \int_{\Omega} e d V+\oint_{\partial \Omega}(e+p) u_{j} n_{j} d S=\oint_{\partial \Omega}\left(\sigma_{i j} u_{i}-q_{j}\right) n_{j} d S
\end{aligned}
$$

where $\Omega$ denotes a control volume, $\partial \Omega$ a surface of the control volume and $n_{j}$ are the component of normal vector to $\partial \Omega$.

\subsection{Implicit Large Eddy Simulation}

Traditional large eddy simulation (LES) is based on the idea of scale separation by spatial filtering which is defined by:

$$
\bar{\phi}=\frac{1}{\Delta_{x} \Delta_{y} \Delta_{z}} \iiint G\left(\frac{x-x^{\prime}}{\Delta_{x}}, \frac{y-y^{\prime}}{\Delta_{y}}, \frac{z-z^{\prime}}{\Delta_{z}}\right) \phi\left(x^{\prime}, y^{\prime}, z^{\prime}\right) d x^{\prime} d y^{\prime} d z^{\prime}
$$

where $G$ denotes the kernel of filter, $\Delta$ the cut-off scale and $\phi$ the primitive variables, which are the density, velocity components, pressure and so on. As the kernel of classical filter, the top hat filter, Gaussian filter and sharp cut-off filter are used frequently. The filtered value by using box filter corresponds to a cell averaged value. In compressible flows, the Favre filtering is used which is filtering operation weighted by the density given as follows:

$$
(\phi)^{F}=\frac{\overline{\rho \phi}}{\bar{\rho}}
$$

The filtered values are called grid scale (GS) and the values that are smaller than cut-off scale are called subgrid scale (SGS). The Favre filtered Navier-Stokes equations, which are the governing equation of compressible LES, are described as follows:

$$
\begin{gathered}
\frac{\partial \bar{\rho}}{\partial t}+\frac{\partial \bar{\rho}\left(u_{j}\right)^{F}}{\partial x_{j}}=0 \\
\frac{\partial \bar{\rho}\left(u_{i}\right)^{F}}{\partial t}+\frac{\partial \bar{\rho}\left(u_{i}\right)^{F}\left(u_{j}\right)^{F}}{\partial x_{j}}+\delta_{i j} \frac{\partial \bar{p}}{\partial x_{j}}-\frac{\partial\left(\sigma_{i j}\right)^{C}}{\partial x_{j}}=\frac{\partial \tau_{i j}}{\partial x_{j}}+\frac{\partial}{\partial x_{j}}\left[\bar{\sigma}_{i j}-\left(\sigma_{i j}\right)^{C}\right] \\
\frac{\partial \bar{\rho}(E)^{F}}{\partial t}+\frac{\partial\left[\bar{\rho}(E)^{F}+\bar{p}\right]\left(u_{j}\right)^{F}}{\partial x_{j}}-\frac{\partial\left(\sigma_{i j}\right)^{C}\left(u_{i}\right)^{F}}{\partial x_{j}}+\frac{\partial\left(q_{j}\right)^{C}}{\partial x_{j}} \\
=-\frac{\partial}{\partial x_{j}}\left[c_{p} Q_{j}+J_{j}-D_{j}-\left\{\bar{q}_{j}-\left(q_{j}\right)^{C}\right\}\right]
\end{gathered}
$$


where $(E)^{F}$ denotes the total energy per unit mass, and we introduce the computable shear-strain tensor $\left(\sigma_{i j}\right)^{C}=2 \mu\left((T)^{F}\right)\left[\left(S_{i j}\right)^{F}-\frac{\delta_{i j}}{3}\left(S_{i j}\right)^{F}\right]$ which depends on the computable rate-of-strain tensor $S_{i j}=\frac{1}{2}\left[\frac{\partial\left(u_{i}\right)^{F}}{\partial x_{j}}+\frac{\partial\left(u_{j}\right)^{F}}{\partial x_{i}}\right]$, and the computable heat flux $\left(q_{j}\right)^{C}=-\kappa\left((T)^{F}\right) \frac{\partial(T)^{F}}{\partial x_{j}}$.

In Equations (77-79), terms in the right hand side are SGS terms. In order to close the equations, the SGS terms in the equations are modelled by SGS models. For incompressible case, only the SGS stress term $\tau_{i j}$ appears in the momentum equation. On the other hand, as can be seen from Equation (79), there are a lot of SGS terms for compressible LES because of the interaction of GS components with SGS components in the filtered energy conservation equation, which are the SGS temperature flux:

the SGS turbulent diffusion:

$$
Q_{j}=\bar{\rho}\left[\left(u_{j} T\right)^{F}-\left(u_{j}\right)^{F}(T)^{F}\right]
$$

$$
J_{j}=\left[\bar{\rho}\left(u_{i} u_{i} u_{j}\right)^{F}-\bar{\rho}\left(u_{i}\right)^{F}\left(u_{i}\right)^{F}\left(u_{j}\right)^{F}-\tau_{i i}\left(u_{j}\right)^{F}\right] / 2
$$

the SGS viscous diffusion:

$$
D_{j}=\overline{\sigma_{i j} u_{i}}-\left(\sigma_{i j}\right)^{C}\left(u_{i}\right)^{F}
$$

and:

$$
\left\{\bar{q}_{j}-\left(q_{j}\right)^{C}\right\}
$$

It is not so efficient to construct all of SGS terms. Implicit LES approaches, on the other hand, do not need any specific SGS models. Implicit LES computation using the Kawamura-Kuwahara scheme was proposed for incompressible flow for the first time by Kawamura and Kuwahara [77]. Then, Boris et al. [78] have proposed the monotonically integrated LES (MILES). In the MILES approach, monotonicity preserving the higher order scheme is used to calculate the nonlinear term. Besides the molecular viscosity, the numerical viscosity inherently involved in the scheme is shown to dissipate the shortest wavelength component of numerical oscillations associated to the given mesh system. As a result, MILES can give a reasonable energy spectrum of homogeneous turbulence with a natural truncation occurring at the maximum wave number. According to the concept of MILES, monotonicity preserving higher order schemes for compressible flows such as the monotone upstream scheme for conservation law (MUSCL) [79], essentially non-oscillatory (ENO) [80,81], weighted ENO (WENO) reconstructions [82,83] or weighted compact nonlinear scheme (WCNS) [84] can be applied. For implicit LES, all SGS terms in Equations (77-79) are neglected and set to be zero.

Recent developments and advances in LES flow modelling are also discussed by Drikakis et al. [85] and Hahn and Drikakis [86]. More specific applications such as bifurcation phenomena in supersonic flows are reflected by Panaras and Drikakis [87]. Recent examples of application of LES techniques to combustion problems can be found in Shimada et al. [88]. 


\subsection{Combustion Model}

In this section, we consider the boundary combustion which is shown in Figure 12. It is assumed that the fuel is pure hydrocarbon compound $\mathrm{C}_{x} \mathrm{H}_{y}$ and the inflow is an air as oxidant. A non-premixed flame in the boundary layer is obtained by the combustion between fuel and oxidant. The chemical reaction is governed by one step reaction as follows:

$$
\mathrm{C}_{x} \mathrm{H}_{y}+\left(x+\frac{y}{4}\right) \mathrm{O}_{2} \rightarrow x \mathrm{CO}_{2}+\frac{y}{2} \mathrm{H}_{2} \mathrm{O}
$$

The five chemical species, $\mathrm{C}_{x} \mathrm{H}_{y}, \mathrm{O}_{2}, \mathrm{CO}_{2}, \mathrm{H}_{2} \mathrm{O}$ and $\mathrm{N}_{2}$, are considered, while $\mathrm{N}_{2}$ does not contribute to the above reaction. For flow includes multi species, the definition of the specific heat $c_{p}[\mathrm{~J} /(\mathrm{kg} \mathrm{K})]$ and the enthalpy $h[\mathrm{~J} / \mathrm{kg}]$ for mixture gas are given respectively by:

$$
\begin{gathered}
c_{p}=\sum_{j} Y_{j} c_{p j} \\
h=\sum_{j} Y_{j} h_{j}
\end{gathered}
$$

where $Y$ denotes the mass fraction and subscript $j$ the $j$-th chemical species. In order to take into account the combustion, the specific heat of each chemical species depends on the temperature strongly. Because the ratio of specific heats $\gamma$ also has the temperature dependency, the pressure cannot be calculated by Equation (67) explicitly. For example, the specific heat at constant pressure $c_{p j}(T)[\mathrm{J} /(\mathrm{kg} \mathrm{K})]$ and enthalpy $h_{j}(T)[\mathrm{J} / \mathrm{kg}]$ for $j$-th chemical species are given by following equations [89]:

$$
\begin{gathered}
\frac{c_{p j}(T)}{R_{j}}=a_{1} T^{-2}+a_{2} T^{-1}+a_{3}+a_{4} T+a_{5} T^{2}+a_{6} T^{3}+a_{7} T^{4} \\
\frac{h_{j}(T)}{R_{j} T}=-a_{1} T^{-2}+a_{2} T^{-1} \ln T+a_{3}+a_{4} \frac{T}{2}+a_{5} \frac{T^{2}}{3}+a_{6} \frac{T^{3}}{4}+a_{7} \frac{T^{4}}{5}+\frac{b_{1}}{T}
\end{gathered}
$$

where $R_{j}[\mathrm{~J} /(\mathrm{kg} \mathrm{K})]$ denotes the gas constant for $j$-th chemical species and gas constant for mixture gas is defined by $R=\sum_{j} Y_{j} R_{j}$. Using the definition of internal energy $\varepsilon=e / \rho-\left(u^{2}+v^{2}+w^{2}\right) / 2=\int c_{v}(T) d T=h(T)-R T$, where $c_{v}(T)[\mathrm{J} /(\mathrm{kg} \mathrm{K})]$ denotes the specific heat at constant volume, the temperature can be calculated by iterative procedure which is, for example, Newton-Raphson method:

$$
f(T)=\{h(T)-R T\}-\left[\frac{e}{\rho}-\frac{1}{2}\left(u^{2}+v^{2}+w^{2}\right)\right]=0
$$

The pressure is obtained by equation of state as follows:

$$
p=\rho n R^{0} T
$$

where $R^{0}=8314.51[\mathrm{~J} /(\mathrm{kg}$ mol K)$)]$ denotes the universal gas constant $n[\mathrm{kgmol} / \mathrm{kg}]$ the mole number per unit mass of mixture gas defined by using the mole number per unit mass of species $n_{j}[\mathrm{kgmol} / \mathrm{kg}]$, as follows: 


$$
n=\sum_{j} n_{j}\left(j=\mathrm{C}_{x} \mathrm{H}_{y}, \mathrm{O}_{2}, \mathrm{H}_{2} \mathrm{O}, \mathrm{CO}_{2} \text { and } \mathrm{H}_{2}\right)
$$

Figure 12. Schematic view of diffusion combustion in a laminar boundary layer on a flat plate.

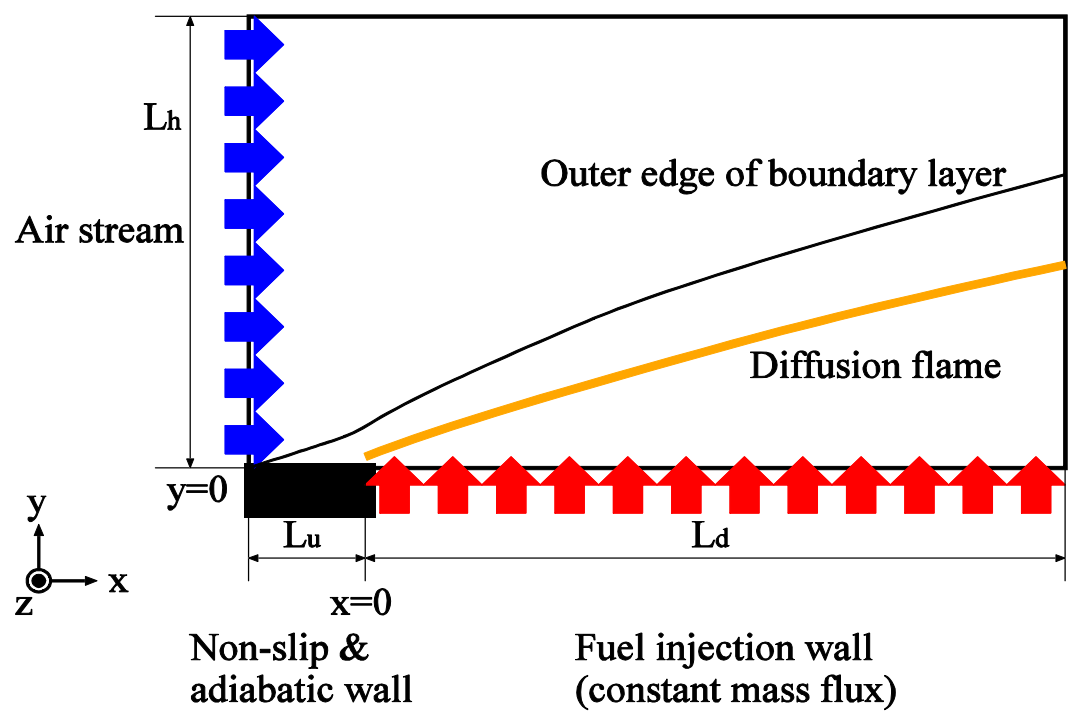

We define $b_{i}^{0}$ as the mole number of element $i(=C, H, O$ and $N)$ per unit mass of mixture gas using $n_{j}$ as follows:

$$
b_{i}^{0}=\sum_{j} a_{i j} n_{j}
$$

These values are the conservative variables unless the velocity between among species is not non-equilibrium. According to Equation (80) with inert gas $\mathrm{N}_{2}$, the Equation (88) is given as follows:

$$
\left[\begin{array}{c}
b_{C}^{0} \\
b_{H}^{0} \\
b_{O}^{0} \\
b_{N}^{0}
\end{array}\right]=\left[\begin{array}{lllll}
x & 0 & 0 & 1 & 0 \\
y & 0 & 2 & 0 & 0 \\
0 & 2 & 1 & 2 & 0 \\
0 & 0 & 0 & 0 & 2
\end{array}\right]\left[\begin{array}{c}
n_{C_{x} H_{y}} \\
n_{O_{2}} \\
n_{\mathrm{H}_{2} \mathrm{O}} \\
n_{\mathrm{CO}_{2}} \\
n_{\mathrm{N}_{2}}
\end{array}\right]=\left[\begin{array}{c}
x n_{C_{x} H_{y}}+n_{\mathrm{CO}_{2}} \\
y n_{C_{x} H_{y}}+2 n_{\mathrm{H}_{2} \mathrm{O}} \\
2 n_{\mathrm{O}_{2}}+n_{\mathrm{H}_{2} \mathrm{O}}+2 n_{\mathrm{CO}_{2}} \\
2 n_{\mathrm{N}_{2}}
\end{array}\right]
$$

The mole number of element $i$ can be expressed by using number density $v_{i}\left[\mathrm{~m}^{-3}\right]$, density of mixture gas $\rho\left[\mathrm{kg} / \mathrm{m}^{3}\right]$ and the Avogadro constant $N_{A}=6.02214179\left[\mathrm{~mol}^{-1}\right]$ as follows:

$$
b_{i}^{0}=\frac{v_{i}}{10^{3} \rho N_{A}}[\mathrm{kgmol} / \mathrm{kg}]
$$

The density of a mixture gas is expressed by:

$$
\rho=\sum_{j} \rho_{j}=\sum_{j} m_{j} v_{j}=\sum_{i} \rho_{i}=\sum_{i} m_{i} v_{i}
$$

where $m_{j}$ and $m_{i}[\mathrm{~kg}]$ denote the mass of $j$-th molecule and the mass of $i$-th element, respectively. For the elements of carbon, hydrogen, oxygen and nitrogen, the masses of element are given by: 


$$
\begin{aligned}
& m_{C}=\frac{12}{N_{A}} \times 10^{-3}[\mathrm{~kg}] \\
& m_{H}=\frac{1}{N_{A}} \times 10^{-3}[\mathrm{~kg}] \\
& m_{O}=\frac{16}{N_{A}} \times 10^{-3}[\mathrm{~kg}] \\
& m_{N}=\frac{14}{N_{A}} \times 10^{-3}[\mathrm{~kg}]
\end{aligned}
$$

The ratio of number density between carbon and hydrogen becomes constant except for in no fuel stream:

$$
v_{C}: v_{H}=x: y
$$

Similarly, the ratio of mole number becomes constant:

$$
b_{C}^{0}: b_{H}^{0}=x: y
$$

On the other hand, when the mass fraction of element $O$ in the air is defined by $A_{O}\left(0 \leq A_{O} \leq 1\right)$, the ratio of number density between oxygen and nitrogen becomes constant except for in no air stream:

$$
v_{O}: v_{N}=b_{O}^{0}: b_{N}^{0}=\frac{A_{O}}{16}: \frac{1-A_{O}}{14}
$$

If the density of mixture gas $\rho$ and one of mole density, for example $v_{C}$, are detected, mole density of $v_{H}, v_{O}$ and $v_{N}$ are also determined by Equations (91), (93) and (95). Then, the mole number of element $b_{i}^{0}$ can be determined by Equation (90).

The transport equation of density of carbon added to solve Navier-Stokes equations is shown as follows, in which one dimension case is considered and the diffusion term is omitted for easy explanation:

$$
\frac{\partial m_{C} v_{C}}{\partial t}+\frac{\partial m_{C} v_{C} u}{\partial x}=0
$$

Using Equations (90) and (92), transport Equation (96) can be rearranged as follows:

$$
\frac{\partial \rho b_{C}^{0}}{\partial t}+\frac{\partial \rho b_{C}^{0} u}{\partial x}=0
$$

From Equation (94), the mole number of hydrogen $b_{H}^{0}$ can be obtained by:

$$
b_{H}^{0}=\frac{y}{x} b_{C}^{0}
$$

And from Equations (90) and (91), the mole number of $b_{O}^{0}+b_{N}^{0}$ is obtained by:

$$
16 b_{O}^{0}+14 b_{N}^{0}=1-12 b_{C}^{0}-b_{H}^{0}
$$


From Equation (95), the mole number of nitrogen $b_{N}^{0}$ is given by:

$$
b_{N}^{0}=\frac{16\left(1-A_{O}\right)}{14 A_{O}} b_{O}^{0}
$$

By substituting Equation (100) into (99), the mole number of oxygen $b_{O}^{0}$ can be obtained. Then, the mole number of nitrogen $b_{N}^{0}$ is calculated by Equation (99) or (100). The boundary conditions which are the fuel injection surface and the inflow boundary are obtained as follows:

$$
\begin{aligned}
& {\left[\begin{array}{l}
b_{C}^{0(1)} \\
b_{H}^{0(1)} \\
b_{O}^{0(1)} \\
b_{N}^{0(1)}
\end{array}\right]=\left[\begin{array}{c}
x n_{C_{x} H_{y}} \\
y n_{C_{x} H_{y}} \\
0 \\
0
\end{array}\right]=\left[\begin{array}{c}
\frac{x}{12 x+y} \\
\frac{y}{12 x+y} \\
0 \\
0
\end{array}\right]} \\
& {\left[\begin{array}{l}
b_{C}^{0(2)} \\
b_{H}^{0(2)} \\
b_{O}^{0(2)} \\
b_{N}^{0(2)}
\end{array}\right]=\left[\begin{array}{c}
0 \\
0 \\
2 n_{O_{2}} \\
2 n_{N_{2}}
\end{array}\right]=\left[\begin{array}{c}
0 \\
0 \\
\frac{A_{O}}{16} \\
\frac{1-A_{O}}{14}
\end{array}\right]}
\end{aligned}
$$

where the superscripts (1) and (2) denote the fuel injection boundary and the air inflow boundary, respectively.

We now define the mixture fraction of fuel $\xi$ as following equation:

$$
\xi \equiv \frac{b_{C}^{0}-b_{C}^{0(2)}}{b_{C}^{0(1)}-b_{C}^{0(2)}}
$$

Then, $b_{C}^{0}$ can be represented by using $\xi$ :

$$
b_{C}^{0}=\left(b_{C}^{0(1)}-b_{C}^{0(2)}\right) \xi+b_{C}^{0(2)}=\frac{x}{12 x+y} \xi
$$

By substitution Equation (104) into (97), the transport Equation (97) can be written by using mixture fraction $\xi$ as follows:

$$
\frac{\partial \rho \xi}{\partial t}+\frac{\partial \rho \xi u}{\partial x}=0
$$


The mole number of each element $\mathrm{C}, \mathrm{H}, \mathrm{O}$ and $\mathrm{N}$ per unit mass of mixture gas are calculated by following equations:

$$
\left[\begin{array}{c}
b_{C}^{0} \\
b_{H}^{0} \\
b_{O}^{0} \\
b_{N}^{0}
\end{array}\right]=\left[\begin{array}{c}
\frac{x}{12 x+y} \xi \\
\frac{y}{12 x+y} \xi \\
\frac{A_{O}}{16}(1-\xi) \\
\frac{1-A_{O}}{14}(1-\xi)
\end{array}\right]
$$

The mole numbers of each species per unit mass $n_{j}$ satisfy the following relations obtained by Equations (89) and (106):

$$
\left[\begin{array}{lllll}
x & 0 & 0 & 1 & 0 \\
y & 0 & 2 & 0 & 0 \\
0 & 2 & 1 & 2 & 0 \\
0 & 0 & 0 & 0 & 2
\end{array}\right]\left[\begin{array}{c}
n_{C_{x} H_{y}} \\
n_{O_{2}} \\
n_{H_{2} O} \\
n_{C_{2}} \\
n_{N_{2}}
\end{array}\right]=\left[\begin{array}{c}
\frac{x}{12 x+y} \xi \\
\frac{y}{12 x+y} \xi \\
\frac{A_{O}}{16}(1-\xi) \\
\frac{1-A_{O}}{14}(1-\xi)
\end{array}\right]
$$

In the above equation, there are more unknown variables than the equations. In order to close the Equation (107), we introduce stoichiometric mixture fraction $\xi_{s t}$ and add some assumption using $\xi_{s t}$. The stoichiometric mixture fraction $\xi_{s t}$ is given by following procedure. According to the chemical reaction Equation (80), the stoichiometric mole ratio of $\mathrm{O}_{2}$ to $\mathrm{C}_{x} \mathrm{H}_{y}$ is $x+y / 4$. Therefore, using Equations (94) and (95), the ratio among the mole numbers per unit mass for each element at the stoichiometric condition is obtained as follows:

$$
b_{C s t}^{0}: b_{H s t}^{0}: b_{O s t}^{0}: b_{N s t}^{0}=x: y: 2 x+\frac{y}{2}:\left(2 x+\frac{y}{2}\right) \frac{16\left(1-A_{O}\right)}{14 A_{O}}
$$

On the other hand, the mole numbers per unit mass for each element satisfy the following equations:

$$
\left[\begin{array}{c}
b_{C s t}^{0} \\
b_{H s t}^{0} \\
b_{O s t}^{0} \\
b_{N s t}^{0}
\end{array}\right]=\left[\begin{array}{c}
\frac{x}{12 x+y} \xi_{s t} \\
\frac{y}{12 x+y} \xi_{s t} \\
\frac{A_{O}}{16}(1-\xi) \\
\frac{1-A_{O}}{14}(1-\xi)
\end{array}\right]
$$

The stoichiometric mixture fraction $\xi_{s t}$ can be calculated by solving Equations (108) and (109):

$$
\xi_{s t}=\frac{(12 x+y) A_{O}}{\left(32+12 A_{O}\right) x+\left(8+A_{O}\right) y}
$$


Let us calculate the mole numbers per unit mass for each species (107) by using the stoichiometric mixture fraction (110). At the condition of $\xi<\xi_{s t}$, it is assumed that there is no fuel after chemical reaction because the amount of fuel is smaller than that of oxidant. This assumption is equivalent to assuming $n_{C_{x} H_{y}}=0$. Then, the Equation (107) can be solved as follows:

$$
\left[\begin{array}{c}
n_{C_{x} H_{y}} \\
n_{\mathrm{O}_{2}} \\
n_{\mathrm{H}_{2} \mathrm{O}} \\
n_{\mathrm{CO}_{2}} \\
n_{\mathrm{N}_{2}}
\end{array}\right]=\left[\begin{array}{c}
0 \\
\frac{1}{32}\left\{A_{O}(1-\xi)-\frac{8(4 x+y)}{12 x+y} \xi\right\} \\
\frac{y}{2(12 x+y)} \xi \\
\frac{x}{12 x+y} \xi \\
\frac{1-A_{O}}{28}(1-\xi)
\end{array}\right]
$$

The mass fraction is defined by:

$$
Y_{j}=n_{j} M_{j} \quad\left(j=\mathrm{C}_{x} \mathrm{H}_{y}, \mathrm{O}_{2}, \mathrm{H}_{2} \mathrm{O}, \mathrm{CO}_{2} \text { and } \mathrm{N}_{2}\right)
$$

where $M_{j}$ denotes the molecular weight. Then, the mass fractions of all species are obtained as follows:

$$
\left[\begin{array}{c}
Y_{C_{x} H_{y}} \\
Y_{\mathrm{O}_{2}} \\
Y_{\mathrm{H}_{2} \mathrm{O}} \\
Y_{\mathrm{CO}_{2}} \\
Y_{\mathrm{N}_{2}}
\end{array}\right]=\left[\begin{array}{c}
0 \\
A_{O}(1-\xi)-\frac{8(4 x+y)}{12 x+y} \xi \\
\frac{9 y}{12 x+y} \xi \\
\frac{44 x}{12 x+y} \xi \\
\left(1-A_{O}\right)(1-\xi)
\end{array}\right]
$$

At the condition of $\xi>\xi_{s t}$, it is similarly assumed that there is no oxidant after chemical reaction because the amount of oxidant is smaller than that of fuel. This assumption is equivalent to assuming $n_{\mathrm{O}_{2}}=0$. Then, the Equation (107) can be solved as follows:

$$
\left[\begin{array}{c}
n_{C_{x} H_{y}} \\
n_{O_{2}} \\
n_{H_{2} O} \\
n_{C O_{2}} \\
n_{N_{2}}
\end{array}\right]=\left[\begin{array}{c}
\frac{8(4 x+y) \xi-A_{O}(1-\xi)(12 x+y)}{8(4 x+y)(12 x+y)} \\
0 \\
\frac{A_{O}(1-\xi) y}{16(4 x+y)} \\
\frac{A_{O}(1-\xi) x}{8(4 x+y)} \\
\frac{1-A_{O}}{28}(1-\xi)
\end{array}\right]
$$


And then, the mass fractions of all species are obtained as follows:

$$
\left[\begin{array}{c}
Y_{C_{x} H_{y}} \\
Y_{O_{2}} \\
Y_{H_{2} O} \\
Y_{\mathrm{CO}_{2}} \\
Y_{N_{2}}
\end{array}\right]=\left[\begin{array}{c}
\xi-\frac{A_{O}(12 x+y)}{8(4 x+y)}(1-\xi) \\
0 \\
\frac{9 A_{O}(1-\xi) y}{8(4 x+y)} \\
\frac{44 A_{O}(1-\xi) x}{8(4 x+y)} \\
\left(1-A_{O}\right)(1-\xi)
\end{array}\right]
$$

At the stoichiometric condition of $\xi=\xi_{s t}$, it is assumed that there is no oxidant and fuel after chemical reaction. This assumption is equivalent assuming $n_{C_{x} H_{y}}=n_{O_{2}}=0$. Then, the Equation (107) can be solved as follows:

$$
\left[\begin{array}{c}
n_{C_{x} H_{y}} \\
n_{O_{2}} \\
n_{H_{2} O} \\
n_{\mathrm{CO}_{2}} \\
n_{N_{2}}
\end{array}\right]=\left[\begin{array}{c}
0 \\
\frac{0}{2\left\{\left(32+12 A_{O}\right) x+\left(8+A_{O}\right) y\right\}} \\
\frac{x A_{O}}{\left(32+12 A_{O}\right) x+\left(8+A_{O}\right) y} \\
\frac{\left(1-A_{O}\right)(32 x+8 y)}{28\left\{\left(32+12 A_{O}\right) x+\left(8+A_{O}\right) y\right\}}
\end{array}\right]
$$

And then the mass fractions of all species are obtained as follows:

$$
\left[\begin{array}{c}
Y_{C_{x} H_{y}} \\
Y_{O_{2}} \\
Y_{H_{2} O} \\
Y_{C_{2}} \\
Y_{N_{2}}
\end{array}\right]=\left[\begin{array}{c}
0 \\
\frac{0}{\left(32+12 A_{O}\right) x+\left(8+A_{O}\right) y} \\
\frac{44 x A_{O}}{\left(32+12 A_{O}\right) x+\left(8+A_{O}\right) y} \\
\frac{\left(1-A_{O}\right)(32 x+8 y)}{\left(32+12 A_{O}\right) x+\left(8+A_{O}\right) y}
\end{array}\right]
$$

Figure 13 shows the profiles of mass fraction of species for methane-air combustion against the mixture fraction at $A_{O}=0.256$.

In the above procedure, the estimated flame temperature becomes very high because intermediate products are not considered and the excessive energy which attribute to the intermediate products is assigned to reactants and product materials. Therefore, intermediate products should be considered for accurate calculation. However, if the number of chemical species increases, the composition cannot be determined by the above procedure because of the lack of equations to solve. 
Figure 13. Mass fraction profiles of each chemical species against mixture fraction $\xi$ for methane and air combustion.

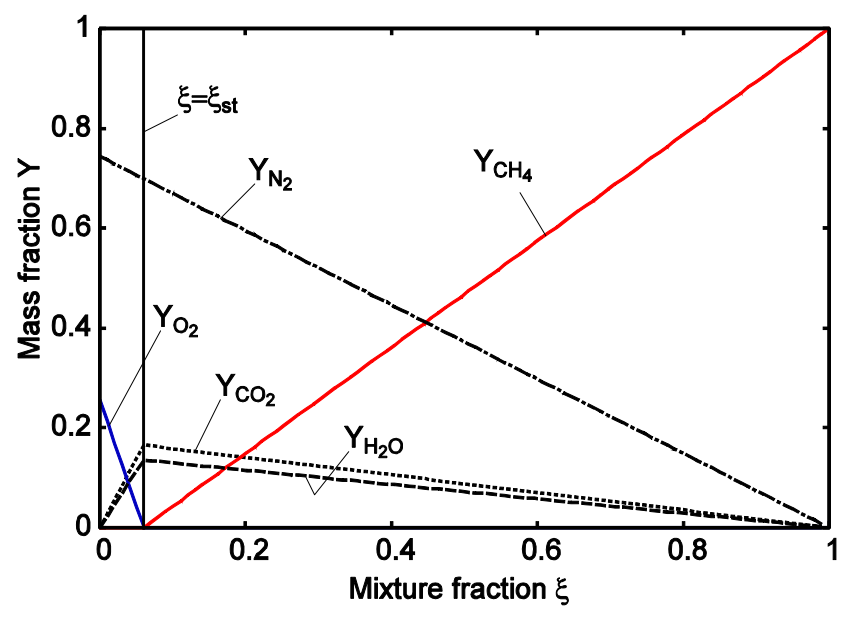

One way of overcoming this problem is to consider the additional chemical reactions with the one step global reaction. For example in this case, we consider the thermal decomposition reactions for carbon dioxide and for water vapor as follows:

$$
\begin{aligned}
& \mathrm{C}_{x} \mathrm{H}_{y}+\left(x+\frac{y}{4}\right) \mathrm{O}_{2} \rightarrow a \mathrm{CO}_{2}+b \mathrm{CO}+c \mathrm{H}_{2} \mathrm{O}+d \mathrm{H}_{2}+e \mathrm{O}_{2} \\
& \mathrm{CO}_{2} \rightarrow \mathrm{CO}+\frac{1}{2} \mathrm{O}_{2}-283.12(\mathrm{~kJ} / \mathrm{kgmol}) \\
& \mathrm{H}_{2} \mathrm{O} \rightarrow \mathrm{H}_{2}+\frac{1}{2} \mathrm{O}_{2}-241.94(\mathrm{~kJ} / \mathrm{kgmol})
\end{aligned}
$$

Using the conservation of elements and law of mass action, chemical composition can be calculated which depends on the pressure, the enthalpy and the fuel mixture fraction. Alternatively, the chemical equilibrium calculation can be used. This procedure is very useful because arbitrary number of chemical species can be treated and specific chemical reaction equations are not needed. The composition after the chemical reaction depends on the pressure, the enthalpy and the fuel mixture fraction for constant pressure process, or the density, the internal energy and the fuel mixture fraction for constant volume process.

\subsection{Numerical Methodology}

For easy explanation, we consider the one dimensional Euler equations in vector form:

$$
\frac{\partial \vec{Q}}{\partial t}+\frac{\partial \vec{E}}{\partial x}=0
$$

where:

$$
\vec{Q}=\left[\begin{array}{c}
\rho \\
\rho u \\
e \\
\rho \xi
\end{array}\right], \vec{E}=\left[\begin{array}{c}
\rho u \\
\rho u^{2}+p \\
(e+p) u \\
\rho \xi u
\end{array}\right]
$$




\subsubsection{Time Integration}

We treat the governing equation as an ordinary differential equation:

$$
\frac{\partial \vec{Q}}{\partial t}=R H S(\vec{Q})
$$

A second-order Runge-Kutta method for the time integration is:

$$
\begin{aligned}
& \vec{Q}^{(1)}=\vec{Q}^{n}+\frac{1}{2} \Delta t R H S\left(\vec{Q}^{n}\right) \\
& \vec{Q}^{n+1}=\vec{Q}^{n}+\Delta t R H S\left(\vec{Q}^{(1)}\right)
\end{aligned}
$$

where superscript $n$ denotes the time step.

\subsubsection{Numerical Flux Function}

Calculating flux functions of governing equations, higher-order difference schemes based on Taylor expansions would not work when discontinuous surface appears in the flowfield, for instance shock waves. For such flowfields, many approximated Riemann solvers have been widely used to calculate numerical flux functions at the cell interface $\vec{E}_{i+1 / 2}$, where subscript $i$ denotes the cell center and midpoint $i+1 / 2$ the cell interface. The Roe's flux difference scheme is given by:

$$
\vec{E}_{i+1 / 2}^{R o e}=\frac{1}{2}\left[\vec{E}\left(\vec{Q}_{R i+1 / 2}\right)+\vec{E}\left(\vec{Q}_{L i+1 / 2}\right)-|\vec{A}|\left(\vec{Q}_{R i+1 / 2}-\vec{Q}_{L i+1 / 2}\right)\right]
$$

where $\vec{A}$ denotes $\partial \vec{E} / \partial \vec{Q}$, and the flux vector splitting schemes are given by:

$$
\vec{E}_{i+1 / 2}^{F V}=\vec{E}^{-}\left(\vec{Q}_{R i+1 / 2}\right)+\vec{E}^{+}\left(\vec{Q}_{L i+1 / 2}\right)
$$

where $\vec{E}^{ \pm}$may be obtained by Steger and Warming's or Van Leer's splitting or the other flux vector splitting methods. The advection upstream splitting method (AUSM), originally developed by Liou and Steffen [90], and its variant AUSM-family schemes can also be used:

$$
\begin{gathered}
\vec{E}_{i+1 / 2}^{A U S M}=\frac{\dot{m}-|\dot{m}|}{2} \vec{\Phi}_{R}+\frac{\dot{m}+|\dot{m}|}{2} \vec{\Phi}_{L}+\widetilde{p} \vec{N} \\
\vec{\Phi}=\left[\begin{array}{c}
1 \\
u \\
H \\
\xi
\end{array}\right], \vec{N}=\left[\begin{array}{c}
0 \\
n_{x} \\
0 \\
0
\end{array}\right]
\end{gathered}
$$

where $H=(e+p) / \rho$ denotes the total enthalpy per unit mass, $n_{x}$ is Cartesian components of a normal vector from the left to the right and $\dot{m}$ represents mass flux, respectively. For one dimensional case, $n_{x}=1$, while, for three dimensional case, $\vec{N}=\left[0, n_{x}, n_{y}, n_{z}, 0,0\right]^{\mathrm{T}}$ and that components satisfy $n_{x}^{2}+n_{y}^{2}+n_{z}^{2}=1$. In Equation (127), $\widetilde{p}$ denotes the corrected pressure term.

In a low Mach number regime, it is well known that almost all of approximated Riemann solvers which are the schemes employed to evaluate numerical flux at cell interface have large numerical 
viscosity. Recently, some low dissipation AUSM-family schemes, AUSM ${ }^{+}$-up [91] and simple low-dissipation AUSM (SLAU) [92] have been developed, which have smaller numerical viscosity not only for high speed region but also for low speed region. In SLAU scheme, corrected pressure $\tilde{p}$ and mass flux $\dot{m}$ are written as follows:

$$
\begin{gathered}
\tilde{p}=\frac{p_{L}+p_{R}}{2}+\frac{\beta_{L}+\beta_{R}}{2}\left(p_{L}-p_{R}\right)+(1-\chi)\left(\beta_{L}+\beta_{R}-1\right) \frac{p_{L}+p_{R}}{2} \\
\dot{m}=\frac{1}{2}\left\{\rho_{L}\left(V_{n L}+\left|\bar{V}_{n}\right|_{L}\right)+\rho_{R}\left(V_{n R}-\left|\bar{V}_{n}\right|_{R}\right)-\frac{\chi}{\bar{c}} \Delta p\right\}
\end{gathered}
$$

where:

$$
\begin{aligned}
& V_{n L / R}=\left\{\begin{array}{cc}
u_{L / R} n_{x} & \text { for 1D case } \\
u_{L / R} n_{x}+v_{L / R} n_{y}+w_{L / R} n_{z} & \text { for 3D case }
\end{array}\right. \\
& M_{L / R}=\frac{V_{n L / R}}{\bar{c}}, \bar{c}=\sqrt{\bar{\gamma} \frac{p_{L}+p_{R}}{\rho_{L}+\rho_{R}}}, \bar{\gamma}=\frac{\gamma_{L}+\gamma_{R}}{2} \\
& \bar{V}_{n}=\frac{\rho_{L}\left|V_{n L}\right|+\rho_{R}\left|V_{n R}\right|}{\rho_{L}+\rho_{R}} \\
& \left|\bar{V}_{n}\right|_{L / R}=(1-g)\left|\bar{V}_{n}\right|+g\left|V_{n L / R}\right| \\
& g=-\max \left[\min \left(M_{L}, 0\right),-1\right] \min \left[\max \left(M_{R}, 0\right), 1\right] \\
& \Delta \rho=\rho_{R}-\rho_{L}, \Delta p=p_{R}-p_{L} \\
& \beta_{L / R}=\left\{\begin{array}{cc}
\frac{1}{4}\left(2 \mp M_{L / R}\right)\left(M_{L / R} \pm 1\right)^{2} & \text { if }\left|M_{L / R}\right|<1 \\
\frac{1}{2}\left(1+\operatorname{sign}\left( \pm M_{L / R}\right)\right) & \text { otherwise }
\end{array}\right. \\
& \chi=(1-\hat{M}), \hat{M}=\left\{\begin{array}{cl}
\min \left(1.0, \frac{1}{\bar{c}} \sqrt{\frac{u_{L}^{2}+u_{R}^{2}}{2}}\right) & \text { for 1D case } \\
\min \left(1.0, \frac{1}{\bar{c}} \sqrt{\frac{u_{L}^{2}+v_{L}^{2}+w_{L}^{2}+u_{R}^{2}+v_{R}^{2}+w_{R}^{2}}{2}}\right) & \text { for 3D case }
\end{array}\right.
\end{aligned}
$$

\subsubsection{High Order Interpolation Method}

For all of approximated Riemann solvers, the spatial accuracy is only first-order when $\vec{Q}_{L i+1 / 2}=\vec{Q}_{i}$ and $\vec{Q}_{R i+1 / 2}=\vec{Q}_{i+1}$. In order to guarantee higher-order spatial accuracy, monotone upstream scheme for conservation law (MUSCL) [79] is used frequently. MUSCL is obtained as follows:

$$
\begin{aligned}
& q_{L i+1 / 2}=q_{i}+\frac{1}{4}\left\{(1+k) \Delta q_{i}+(1-k) \Delta q_{i-1}\right\} \\
& q_{R i+1 / 2}=q_{i+1}-\frac{1}{4}\left\{(1+k) \Delta q_{i}+(1-k) \Delta q_{i+1}\right\}
\end{aligned}
$$


where $q$ denotes primitive variables and $\Delta q_{i}=q_{i+1}-q_{i}$. The parameter $k$ decides the spatial accuracy of the scheme shown in Table 13.

Table 13. Spatial accuracy of MUSCL interpolation for several values of parameter $k$.

\begin{tabular}{ll}
\hline$k$ & Scheme \\
\hline-1 & second-order upwind \\
0 & second-order \\
$1 / 3$ & third-order upwind \\
$1 / 2$ & QUICK (second-order upwind) \\
1 & second-order central \\
\hline
\end{tabular}

In order to give TVD property, the flux limiter $\phi\left(r_{i}\right)$ is introduced as follows:

$$
\begin{aligned}
& q_{L i+1 / 2}=q_{i}+\frac{\phi\left(r_{i}\right)}{4}\left\{(1+k) \Delta q_{i}+(1-k) \Delta q_{i-1}\right\} \\
& q_{R i+1 / 2}=q_{i+1}-\frac{\phi\left(r_{i+1}\right)}{4}\left\{(1+k) \Delta q_{i}+(1-k) \Delta q_{i+1}\right\}
\end{aligned}
$$

where:

$$
r_{i}=\frac{\Delta q_{i-1}}{\Delta q_{i}}
$$

Three famous examples of flux limiter will be shown below.

Minmod limiter [93]:

$$
\phi(r)=\max [0, \min (1, r)]
$$

Superbee limiter [93]:

$$
\phi(r)=\max [0, \min (1, \beta r), \min (r, \beta)],(1 \leq \beta \leq 2)
$$

Van Leer limiter [94]:

$$
\phi(r)=\frac{r+|r|}{1+|r|}
$$

Alternatively, the slope limiter function $S_{i}$ which is given by Anderson et al. [95] can be used:

$$
\begin{gathered}
q_{L i+1 / 2}=q_{i}+\frac{S_{i}}{4}\left\{\left(1+k S_{i}\right) \Delta q_{i}+\left(1-k S_{i}\right) \Delta q_{i-1}\right\} \\
q_{R i+1 / 2}=q_{i+1}-\frac{S_{i+1}}{4}\left\{\left(1+k S_{i+1}\right) \Delta q_{i}+\left(1-k S_{i+1}\right) \Delta q_{i+1}\right\} \\
S_{i}=\frac{2 \Delta q_{i} \Delta q_{i-1}+\varepsilon}{\left(\Delta q_{i}\right)^{2}+\left(\Delta q_{i-1}\right)^{2}+\varepsilon}
\end{gathered}
$$

where $\varepsilon=10^{-6}$ is introduced to avoid the denominator becoming zero. 


\subsection{Non-Premixed Flame in Three-Dimensional Flowfield}

\subsubsection{Brief Description for Computation}

In this section, we compute the methane and air combustion in a three-dimensional flowfield. The one step global reaction (80) is assumed. The governing equations are discretized by the cell centered finite volume method. In order to calculate the numerical flux functions, SLAU is used. In order to guarantee the third order spatial accuracy with TVD, MUSCL interpolation is introduced to reconstruct a distribution of variables from cell centered values. The viscous flux is calculated by second order central difference scheme and two-stage Runge-Kutta method is used to evaluate the time integration. A CFL number of 0.5 is assumed. Newton-Raphson method is used to obtain temperature value from total energy. In this calculation, the turbulent boundary layer is predicted by not using any explicit turbulent models. It is expected that the numerical viscosity of higher order numerical scheme plays a role of turbulent viscosity in the subgrid scale. For present simulation of combustion, the influence of scalar dissipation rate is ignored while this assumption is not appropriate modeling for computation of turbulent diffusion flame. This indicates that the extinction of flame cannot be considered in the present calculation. For the combustion model, flame sheet model is used. The parallel computation using Open MP is carried out using 16 vector processors on the JAXA Supercomputer System (JSS) installed at Aerospace Research and Development Directorate (ARD), Japan Aerospace Exploration Agency (JAXA).

The initial flowfield is given by assuming two-dimensional solution at each spanwise location. The temperature contours of initial two-dimensional solution are shown in Figure 14. For three-dimensional calculation, the total computational domain is $x \in[-0.02,5.0], y \in[0,0.3]$ and $z \in[-0.135,0.135]$. The main region is the domain $x \in[-0.02,1.0]$ and $y \in[0,0.5]$ which is inner part of red rectangle shown in Figure 14, and outer part of that is buffer region.

Figure 14. Initial temperature contours in the cross section at $z=0 \mathrm{~m}$.

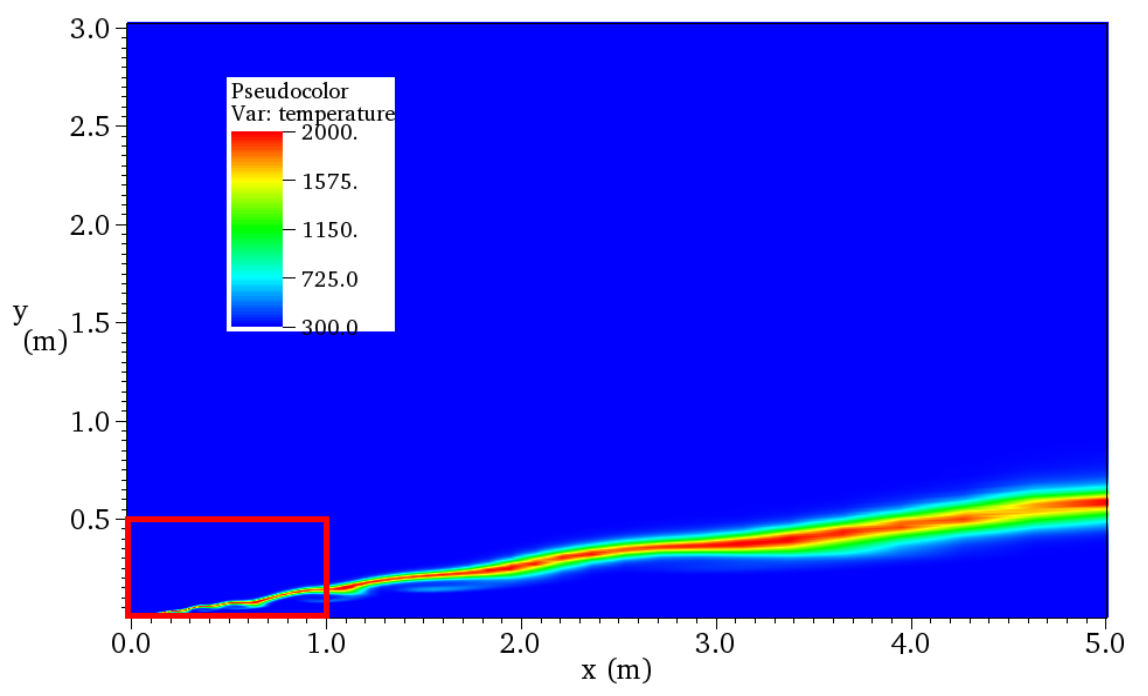

A non-slip adiabatic wall boundary condition is enforced along the wall surface for $x<0$, and fuel injection with constant mass flow rate is considered for $x \geq 0$. It is assumed that the injection velocity takes the value $0.4 \mathrm{~m} / \mathrm{s}$ when static temperature and static pressure are $300 \mathrm{~K}$ and $1.0 \mathrm{~atm}$, respectively. 
As the inflow, free stream of air is assumed and that velocity is $10.0 \mathrm{~m} / \mathrm{s}$. The static pressure and the static temperature at the infinite distance are assumed as $p_{\infty}=1.0 \mathrm{~atm}$ and $T_{\infty}=300 \mathrm{~K}$, respectively. The Riemann boundary condition is employed along the inflow and upper boundary. Along the outlet boundary, a zeroth extrapolation is used to give the dependent variables. At both side walls of the computational domain, periodic boundary conditions are enforced. The total number of grid points in the computational domain is $253 \times 182 \times 151$ in the streamwise direction, normal direction to the wall surface and spanwise direction, respectively.

\subsubsection{Results and Discussions}

Figure 15 shows the typical contours of instantaneous temperature in the cross section at $z=0 \mathrm{~m}$. It can be found that the two-dimensional vortices are induced in the shear layer because of Kelvin-Helmholtz instability, and this indicates that the mixing of fuel and oxidizer ant the shear layer is promoted.

Figure 15. Typical instantaneous temperature contours in the cross section at $z=0 \mathrm{~m}$.

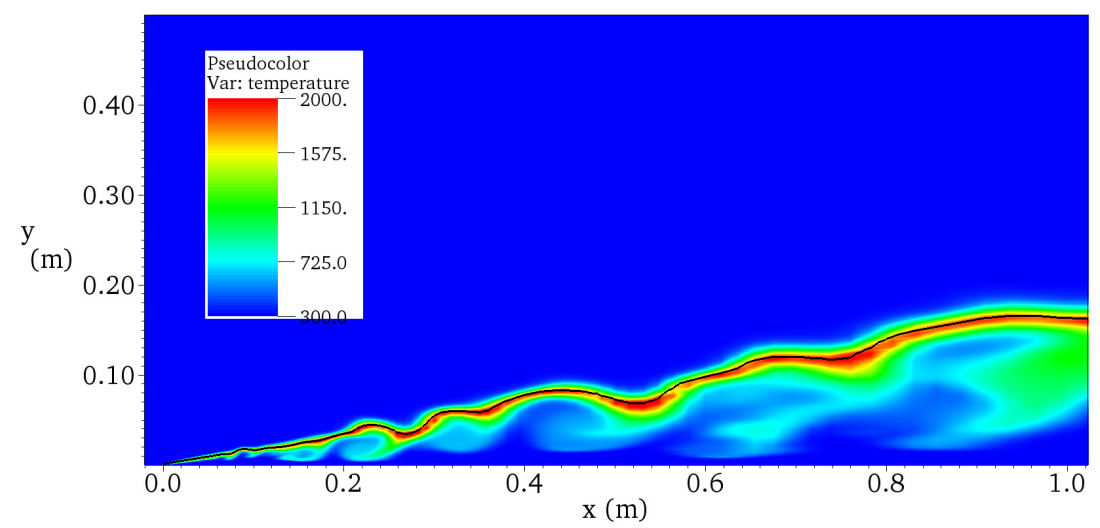

The comparison of iso-surface of mixture fractions are shown in Figure 16a,b, which are the flowfield at $t=0 \mathrm{~s}$ and at $t=0.536 \mathrm{~s}$, respectively.

Figure 16. Iso-surfaces of mixture fractions. (a) $t=0 \mathrm{~s}$; (b) $t=0.536 \mathrm{~s}$.

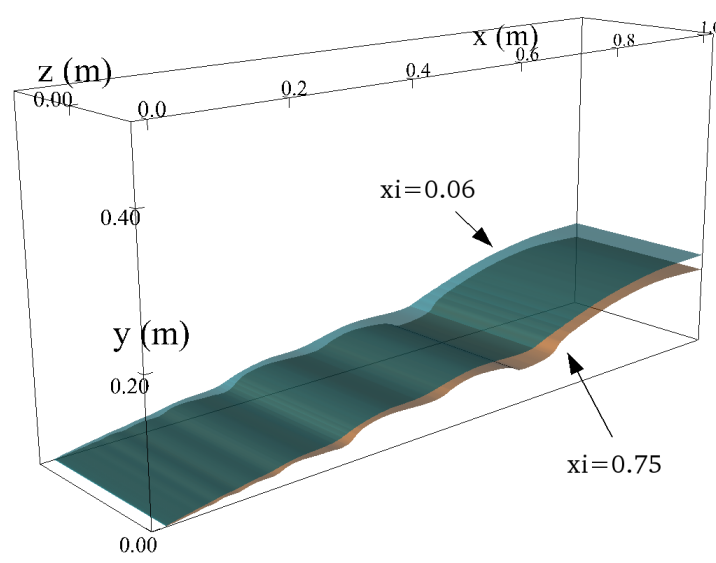

(a)

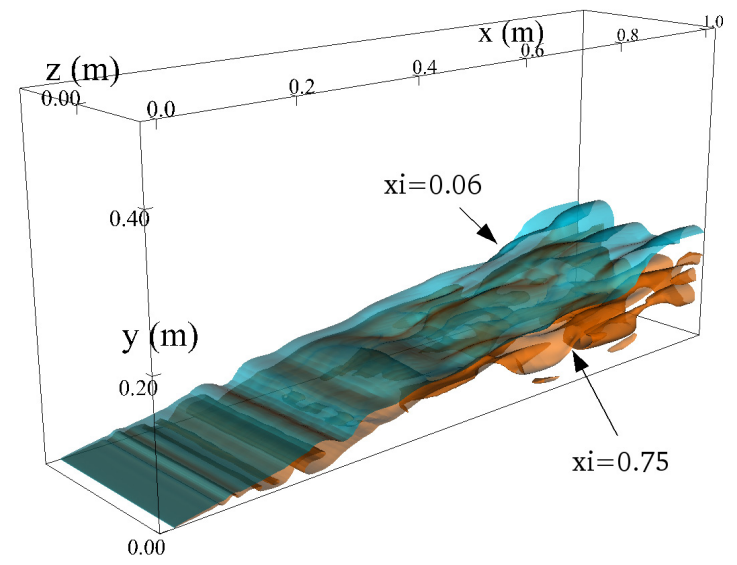

(b) 
The green iso-surface denotes $\xi=\xi_{s t} \cong 0.06$ indicating flame location, and the yellow one denotes $\xi=0.75$ which is one of iso-surface in the fuel stream. In Figure $16 \mathrm{~b}$, the three-dimensionality of iso-surfaces appears obviously by comparing those in Figure 16a, however, this cannot indicate that the turbulent flow has developed because the computation time is insufficient.

Figure $17 \mathrm{a}-\mathrm{c}$ show the temperature contours, contours of mass fraction of products $Y_{\mathrm{CO}_{2}}+\mathrm{H}_{2} \mathrm{O}$ and the projected velocity vectors, respectively, on the cross section at $x=0.9 \mathrm{~m}$ where the mixing is promoted. These figures are obtained at the same instance with Figure 16b. In these figures, one can find that the high temperature region corresponds to the region where the large amount of products exists. This high temperature gas of combustion products is convected toward fuel stream side due to the vortices induced by the Kelvin-Helmholtz instability in the shear layer. Again, the turbulent flow has not yet developed in this instance while it can be found that the longitudinal vortices appear in the fuel stream.

Figure 18 shows the mole number in the main computational domain normalized by the initial state values. In Figure 18, fuel denotes $\mathrm{CH}_{4}$, oxidizer $\mathrm{O}_{2}+\mathrm{N}_{2}$ and products $\mathrm{CO}_{2}+\mathrm{H}_{2} \mathrm{O}$, respectively. In this figure, one can find that the fuel is consumed and the products increase about twice as much as initial value as time advances. This is because a large number of products near the diffusion flame are convected and chemical reaction is promoted.

In order to examine the influence of grid resolution, the results are compared with those obtained by computation using coarser grid. The total grid number of coarser grid is $201 \times 151 \times 101$ in the streamwise direction, normal direction to the wall surface and spanwise direction, respectively. The parallel computation using Open MP is carried out using 8 vector processors. The comparison of computation costs between finer grid and coarser grid is summarized in Table 14.

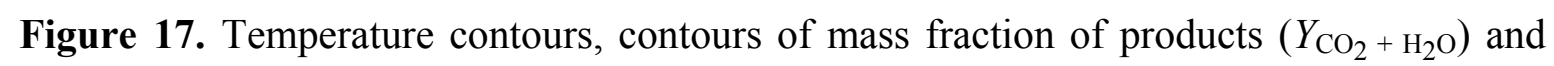
projected velocity vectors on the cross section at $x=0.9 \mathrm{~m}$. (a) Temperature; (b) Mass fraction of products; (c) Projected velocity field.

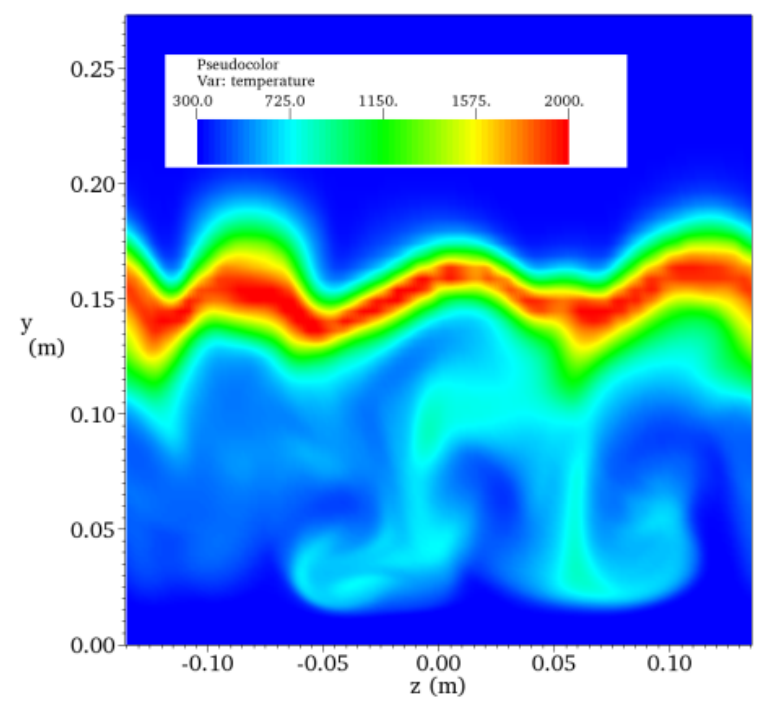

(a)

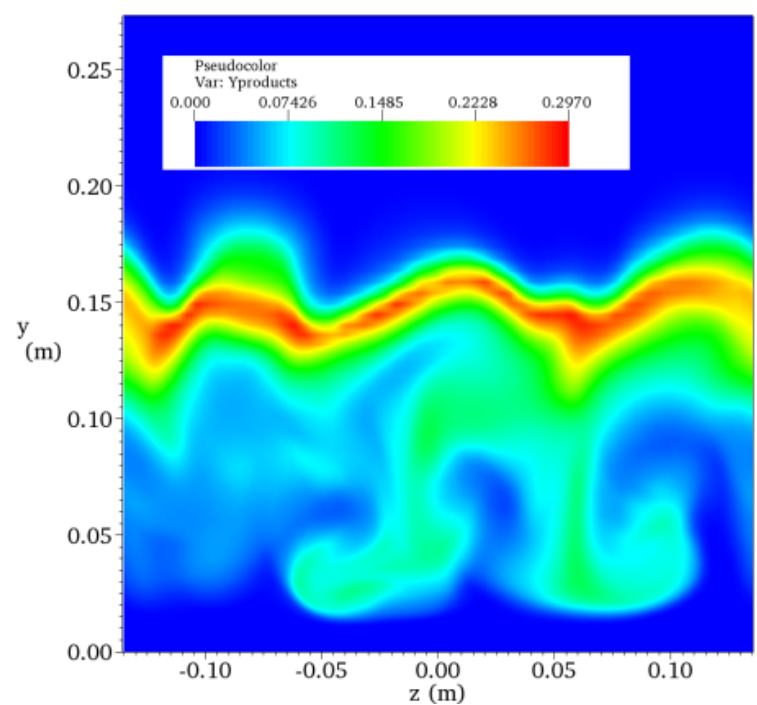

(b) 
Figure 17. Cont.

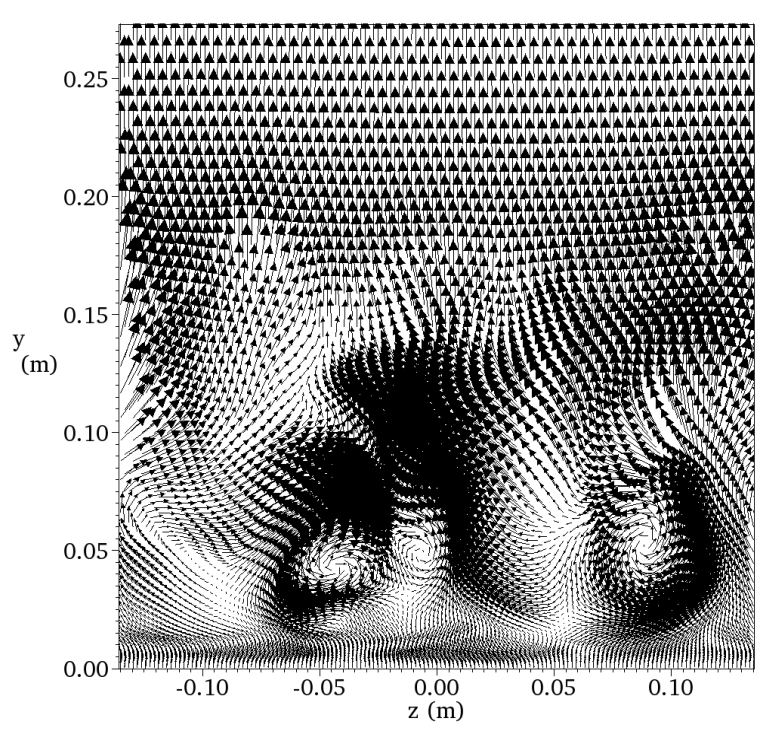

(c)

Figure 18. Comparison of mole number normalized by initial values.

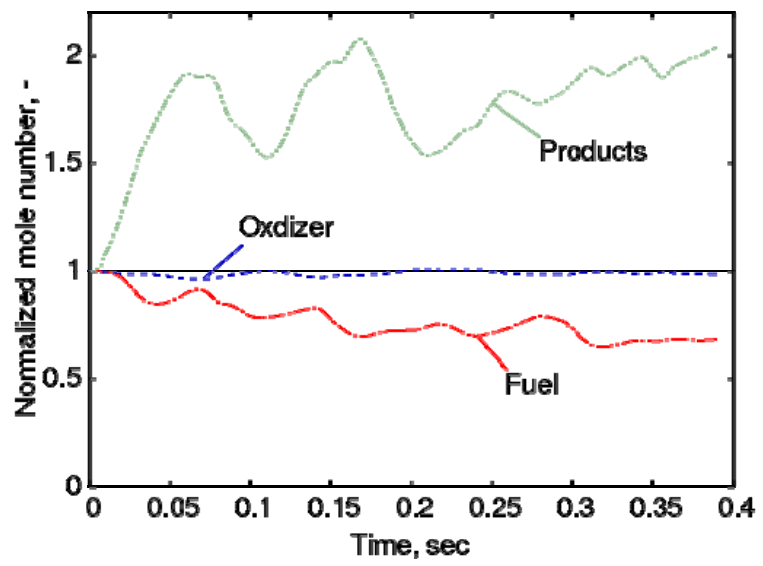

Figures 19 and 20 show the contours of instantaneous temperature in the cross section at $z=0 \mathrm{~m}$ and the iso-surfaces of mixture fraction at $t=0.536 \mathrm{~s}$, respectively, which are obtained by using coarser grid. In these figures, the two-dimensional vortices induced by Kelvin-Helmholtz instability can be seen near the leading edge of fuel injection wall and three-dimensionality appears downstream side in the flowfield. These features are qualitatively the same as those obtained by finer grid shown in Figure 15 and 16b. Figure 21 shows the comparison of mole number normalized by initial values obtained in coarse grid computation. The mole number of products becomes about twice and that of fuel decreases down to $60 \%$ of initial values, respectively. The mole number of oxidizer does not change so much. The features are consistent with those obtained by finer grid in Figure 18.

Table 14. Comparison of computation costs.

\begin{tabular}{cccc}
\hline Resolution & Grid Points & CPU Numbers & Total CPU Times per 10 $\mathbf{4}^{\mathbf{4}}$ Time Steps \\
\hline Fine & $253 \times 182 \times 151$ & 16 & $10.64 \mathrm{~h}$ \\
Coarse & $201 \times 151 \times 101$ & 8 & $5.34 \mathrm{~h}$ \\
\hline
\end{tabular}


Figure 19. The instantaneous temperature contours in the cross section at $z=0 \mathrm{~m}$, $t=0.536 \mathrm{~s}$ obtained by using coarser grid.

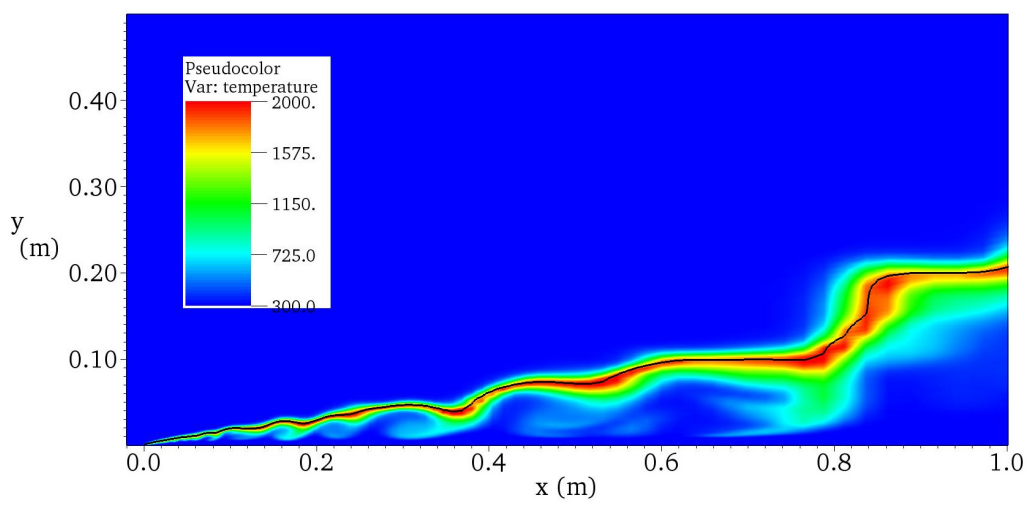

Figure 20. The iso-surfaces of the mixture fraction obtained by using coarser grid.

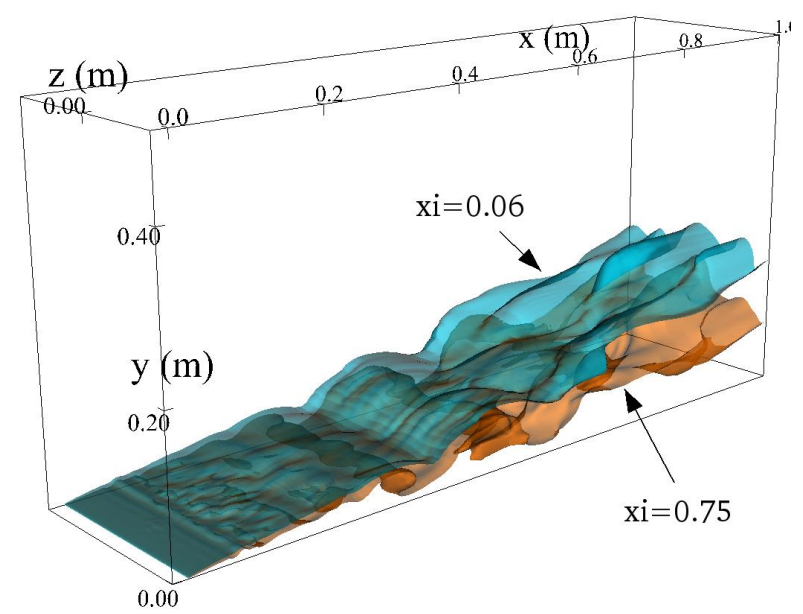

Figure 21. Comparison of mole number normalized by initial values obtained by using coarser grid.

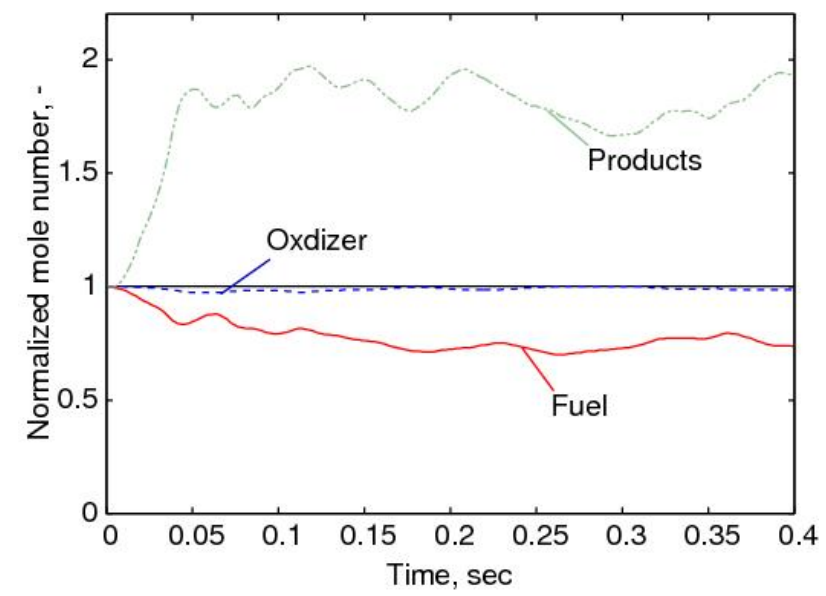

\section{Conclusions}

The main objective of the paper has been review of fundamental problems related to further polymer fuel application in Hybrid Rocket Engines. Fundamental flammability characteristics of 
polymeric fuels have been discussed. Success of polymer fuel applications to Hybrid Propulsion depends on further improvement of their combustion characteristics. In the context of hybrid propulsion, this effectively means enhancement of regression (and therefore burning) rates. This problem is not an easy one since estimations suggest that regression rate needs be increased dramatically (possibly by an order of magnitude). Chemical fuel modification is one promising route to overcoming the problem. Some suggestions for potential modifications of basic polymer fuels have been presented.

There is no doubt that chemical modification of polymers requires substantial development of experimental techniques. For that reason, a number of existing and potential techniques have been discussed. A large part of the paper is devoted to mathematical modelling of combustion process in Hybrid Rocket Engines. Critical models that require further development and implementation have been discussed. Among them are combustion (essentially based on flamelet approach), radiation, solid fuel regression and injector flow models. The problem of accurate modelling of thermal degradation of polymeric fuel and its subsequent combustion has been identified. This is potentially the most significant obstacle on the way towards accurate CFD modelling of Hybrid combustion, since turbulent flow and combustion models are relatively well developed at the moment. Supplying input kinetic data to combustion models, on the other hand, represent a significant challenge. Such input requires detailed knowledge of pyrolysis gas composition, and subsequently, detailed information on combustion kinetics of major products. For typical polymeric fuels, estimation of major thermal decomposition products and their combustion kinetics has been done based on available data. In reality, much more accurate data would be needed. In this direction, a set-up for evolved gas analysis has been proposed. This technique, although complicated, has a capability of delivering required information on pyrolysis gas composition.

The last section of the paper presents results of preliminary 3-D CFD calculations, based on Implicit Large Eddy simulations. At the moment, one step global reaction with prescribed fuel injection rate is used. Combustion is modelled via a fast chemistry approach. Calculations demonstrate that implicit LES is able to provide very detailed information on flow field and combustion in a boundary layer. Basic algorithms have been described, and it has been demonstrated that they properly predict essential features of the phenomenon. The technique, based on solution of fully compressible flow model is quite expensive, however, future increase in computational power warrants its practical application.

\section{Acknowledgements}

The first author wishes to acknowledge support from Japan Aerospace Exploration Agency (JAXA) for his visit as Visiting Professor to JAXA in 2009. The second author acknowledges utilization of some basic research facilities, obtained through the UK's Engineering and Physical Sciences Research Council fund (grant number: EP/F068867/1), awarded to him. Research of the third and fourth authors has been supported, in part, by the Hybrid Rocket Research Working Group (HRrWG) of the Institute of Space and Astronautical Science, Japan Aerospace Exploration Agency. These authors wish to thank members of HRrWG for their helpful discussions. 


\section{References}

1. Chiaverini, M.J.; Kuo, K.K. Fundamentals of Hybrid Rocket Combustion and Propulsion (Progress in Astronautics and Aeronautics); AIAA: Reston, VA, USA, 2007.

2. Altman, D.; Holzman, A. Overview and history of hybrid rocket propulsion. In Fundamentals of Hybrid Rocket Combustion and Propulsion (Progress in Astronautics and Aeronautics); Chiaverini, M.J., Kuo, K.K., Eds.; AIAA: Reston, VA, USA, 2007; Volume 218, Chapter 1, pp. 1-36.

3. Cullis, C.F.; Hirschler, M.M. The Combustion of Organic Polymers; Clarendon Press: Oxford, UK, 1981.

4. Joseph, P.; Ebdon, J.R. Recent developments in flame-retarding thermoplastics and thermosets. In Fire Retardant Materials; Horrocks, A.R., Price, D., Eds.; Woodhead Publishing Limited: Cambridge, UK, 2000; pp. 220-263.

5. Arnold, C., Jr. Stability of high temperature polymers. J. Polym. Sci. Macromol. Rev. 1979, 14, 265-378.

6. Martel, B. Charring process in thermoplastic polymers: Effect of condensed phase oxidation on the formation of chars in pure polymers. J. Appl. Polym. Sci. 1988, 35, 1213-1226.

7. Fenimore, C.P.; Martin, F.J. Flammability of polymers. Combust. Flame 1966, 10, 135-139.

8. Babrauskas, V. Development of a cone calorimeter - a bench-scale heat release apparatus cased on oxygen consumption. Fire Mater. 1984, 8, 81-95.

9. Schartel, B.; Bartholmai, M.; Knoll, U. Some comments on the use of cone calorimetric data. Polym. Degrad. Stab. 2005, 88, 540-547.

10. De Ris, J.L.; Khan, M.M. A sample holder for determining material properties. Fire Mater. 2000, 24, 219-226.

11. Lyon, R.E.; Walters, R.N. Pyrolysis combustion flow calorimetry. J. Anal. Appl. Pyrolysis 2004, $71,27-46$.

12. Cogen, J.M.; Lin, T.S.; Lyon, R.E. Correlations between pyrolysis flow combustion calorimetry and conventional flammability tests with halogen-free flame retardant polyolefin compounds. Fire Mater. 2009, 33, 33-50.

13. Ebdon, J.R.; Hunt, B.J.; Jones, M.S.; Thorpe, F.G. Chemical modification of polymers to improve flame retardance-II: The influence of silicon-containing groups. Polym. Degrad. Stab. 1996, 69, 395-400.

14. Ebdon, J.R.; Guisti, L.; Hunt, B.J.; Jones, M. The effects of some transition-metal compounds on the flame retardance of poly(styrene-co-4-vinyl pyridine) and poly(methyl methacrylate-co-4vinyl pyridine). Polym. Degrad. Stab. 1998, 60, 401-407.

15. Armitage, P.; Ebdon, J.R.; Hunt, B.J.; Jones, M.S.; Thorpe, F.G. Chemical modification of polymers to improve flame retardance-I. The influence of boron-containing groups. Polym. Degrad. Stab. 1996, 54, 387-393.

16. Ebdon, J.R.; Price, D.B.; Hunt, B.J.; Joseph, P.; Gao, F.; Milnes, G.J.; Cunliffe, L.K. Flame retardance in some polystyrenes and poly(methyl methacrylate)s with covalently bound phosphorus-containing groups: initial screening experiments and some laser pyrolysis mechanistic studies. Polym. Degrad. Stab. 2000, 69, 267-277. 
17. Ebdon, J.R.; Hunt, B.J.; Joseph, P. Thermal degradation and flammability characteristics of some polystyrenes and poly(methyl methacrylate)s chemically modified with silicon-containing groups. Polym. Degrad. Stab. 2004, 83, 181-185.

18. Zhang, S.; Hull, T.R.; Horrocks, A.R.; Smart, G.; Kandola, B.K.; Ebdon, J.; Hunt, B.; Joseph, P. Thermal degradation analysis and XRD characterisation of fibre-forming synthetic polypropylene containing nanoclay. Polym. Degrad. Stab. 2007, 92, 727-732.

19. Marxman, G.A.; Gilbert, M. Turbulent boundary layer combustion in the hybrid rocket. In Ninth International Symposium on Combustion; Academic Press: New York, NY, USA, 1963; pp. 371-383.

20. Marxman, G.A.; Wooldridge, C.E.; Muzzy, R.J. Fundamentals of hybrid boundary layer combustion. In Heterogeneous Combustion (AIAA Progress in Astronautics and Aeronautics); Wolfhard, H.G., Glassman, I., Green, L., Jr.; Eds.; Academic Press: New York, NY, USA, 1964; Volume 15, pp. 485-521.

21. Chiaverini, M.J. Review of solid-fuel regression rate behavior in classical and nonclassical hybrid rocket motors. In Fundamentals of Hybrid Rocket Combustion and Propulsion (Progress in Astronautics and Aeronautics); Chiaverini, M.J., Kuo, K.K., Eds.; AIAA: Reston, VA, USA, 2007; Volume 218, pp. 37-125.

22. Lengelle, G. Solid-fuel pyrolysis phenomena and regression rate. In Fundamentals of Hybrid Rocket Combustion and Propulsion (Progress in Astronautics and Aeronautics); Chiaverini, M.J., Kuo, K.K., Eds.; AIAA: Reston, VA, USA, 2007; Volume 218, pp. 127-165.

23. Zeng, W.R.; Li, S.F.; Chow, W.K. Review on chemical reactions of burning poly(methylmethacrylate) PMMA. J. Fire Sci. 2002, 20, 401-433.

24. Ananth, R.; Ndubizu, C.C.; Tatem, P.A. Burning rate distributions for boundary layer flow combustion of a PMMA plate in forced flow. Combust. Flame 2003, 135, 35-55.

25. Stoliarov, S.I.; Crowley, S.; Lyon, R.E.; Linteris, G.T. Prediction of the burning rates of non-charring polymers. Combust. Flame 2009, 156, 1068-1083.

26. Arisawa, H.; Brill, T.B. Kinetics and mechanisms of flash pyrolysis of poly(methyl methacrylate) (PMMA). Combust. Flame 1997, 109, 415-426.

27. Bedir, H.; T'ien, J.S. A Computational Study of Flame Radiation in PMMA Diffusion Flames Including Fuel Vapor Participation. In Proceedings of the Twenty-Seventh Symposium (International) on Combustion, Combustion Institute, Pittsburgh, PA, USA, 2-7 August 1998; Volume 27, pp. 2821-2828.

28. Vovelle, C.; Delfau, J.L.; Reuillon, M.; Bransier, J.; Laraqui, N. Experimental and numerical study of the thermal degradation of PMMA. Combust. Sci. Technol. 1987, 53, 187-207.

29. Krishnamurthy, L.; Williams, F.A. Fourteenth Symposium (International) on Combustion; The Combustion Institute: Pittsburgh, PA, USA, 1974.

30. Kashiwagi, T.H.; Brown, J.E. Thermal and oxidative degradation of poly(methyl methacrylate) molecular weight. Macromolecules 1985, 18, 131-138.

31. Kumar, R.N.; Stickler, D.B. Polymer-degradation theory of pressure-sensitive hybrid combustion. Proc. Symp. (Int.) Combust. 1971, 13, 1059-1072.

32. Madorsky, S.L. Thermal Degradation of Organic Polymers; Interscience Publishers: New York, NY, USA, 1964. 
33. Zeng, W.R.; Li, S.F.; Chow, W.K. Preliminary studies on burning behavior of poly(methylmethacrylate) (PMMA). J. Fire Sci. 2002, 20, 297-317.

34. GRI-MECH Database Homepage. Available Online: http://www.me.berkeley.edu/gri-mech/ (accessed on 21 October 2011)

35. Bell, K.M.; Tipper, C.F.H. The slow combustion of methylalcohol, a general investigation. Proc. R. Soc. Lond. Ser. A 1956, 238, 256-268.

36. Vardanyan, I.A.; Sachyan, G.A.; Nalbandyan, A.B. Kinetics and mechanism of formaldehyde oxidation. Combust. Flame 1971, 17, 315-322.

37. Hay, J.M.; Hessam, K. The oxidation of gaseous formaldehyde. Combust. Flame 1971, 16, 237-242.

38. Hidaka, Y.; Hattori, K.; Okuno, T.; Inami, K.; ABE, T.; Koike, T. Shock-tube and modeling study of acetylene pyrolysis and oxidation. Combust. Flame 1996, 107, 401-417.

39. Wilkie, C.A. TGA/FTIR: An extremely useful technique for studying polymer degradation. Polym. Degrad. Stab. 1999, 66, 301-306.

40. Raemaekers, K.G.H.; Bart, J.C.J. Applications of simultaneous thermogravimetry-mass spectrometry in polymer analysis. Thermochim. Acta 1997, 295, 1-58.

41. Kaisersberger, E.; Post, E. Practical aspects for the coupling of gas analytical methods with thermal-analysis instruments. Thermochim. Acta 1997, 295, 73-93.

42. Maciejewski, M.; Baiker, A. Quantitative calibration of mass spectrometric signals measured in coupled TA-MS system. Thermochim. Acta 1997, 295, 95-105.

43. Marsanich, K.; Barontini, F.; Cozzani, V.; Petarca, L. Advanced pulse calibration techniques for the quantitative analysis of TG-FTIR data. Thermochim. Acta 2002, 390, 153-168.

44. Branley, N.; Jones, W.P. Large eddy simulation of a turbulent non- premixed flame. Combust. Flame 2001, 127, 1914-1934.

45. Peters, N. Laminar diffusion flamelet models in non-premixed turbulent combustion. Prog. Energy Combust. Sci. 1984, 10, 319-339.

46. Pitsch, H.; Chen, M.; Peters, N. Unsteady flamelet modeling of turbulent hydrogen-air diffusion flames. Proc. Symp. (Int.) Combust. 1998, 27, 1057-1064.

47. Pitsch, H.; Cha, C.M.; Fedotov, S. Interacting Flamelet Model for Non-Premixed Turbulent Combustion with Local Extinction and Re-Ignition; Annual Research Briefs 2001; Center for Turbulence Research, Stanford University: Menlo Park, CA, USA, 2001.

48. Pitsch, H.; Peters, N. A consistent flamelet formulation for non-premixed combustion considering differential diffusion effects. Combust. Flame 1998, 114, 26-40.

49. Gran, I.R.; Melaaen, M.C.; Magnussen, B.F. Numerical simulation of local extinction effects in turbulent combustor flows of methane and air. Proc. Symp. (Int.) Combust. 1994, 25, 1283-1291.

50. Pantano, C.; Sarkar, S.; Williams, F.A. Mixing of a conserved scalar in a turbulent reacting shear layer. J. Fluid Mech. 2003, 481, 291-328.

51. Pitsch, H. Extended Flamelet Model for LES of Non-Premixed Combustion; Annual Research Briefs 2000; Center for Turbulence Research, Stanford University: Menlo Park, CA, USA, 2000.

52. Chiaverini, M.J.; Serin, N.; Johnson, D.K.; Lu, Y.; Kuo, K.K.; Risha, G.A. Regression rate behavior of hybrid rocket solid fuels. J. Propul. Power 2000, 16, 125-132. 
53. Sankaran, V. Computational fluid dynamics modeling of hybrid rocket flowfields. In Fundamentals of Hybrid Rocket Combustion and Propulsion (Progress in Astronautics and Aeronautics); Chiaverini, M.J., Kuo, K.K., Eds.; AIAA: Reston, VA, USA, 2007; Volume 218, pp. 323-349.

54. Hossain, M.; Jones, J.C.; Malalasekera, W. Modelling of a bluff-body nonpremixed flame using a coupled radiation/flamelet combustion model. Flow Turbul. Combust. 2001, 67, 217-234.

55. Novozhilov, V. Computational fluid dynamics modelling of compartment fires. Prog. Energy Combust. Sci. 2001, 27, 611-666.

56. Lockwood, F.C.; Shah, N.G. A new radiation solution method for incorporation in general combustion prediction procedures. Proc. Symp. (Int.) Combust. 1981, 18, 1405-1414.

57. Novozhilov, V.; Harvie, D.J.E.; Kent, J.H.; Apte, V.B.; Pearson, D. A computational fluid dynamics study of wood fire extinguishment by water sprinkler. Fire Saf. J. 1997, 29, 259-282.

58. Novozhilov, V.; Harvie, D.J.E.; Green, A.R.; Kent, J.H. A computational fluid dynamic model of fire burning rate and extinction by water sprinkler. Combust. Sci. Technol. 1997, 123, 227-245.

59. Kuo, K.K. Principles of Combustion; Wiley: New York, NY, USA, 1986.

60. Gosman, A.D.; Ioannides, E. Aspects of computer simulation of liquid-fuelled combustors. In Proceedings of the American Institute of Aeronautics and Astronautics, Aerospace Sciences Meeting, St. Louis, MO, USA, 12-15 January 1981.

61. Faeth, G.M. Evaporation and combustion of sprays. Prog. Energy Combust. Sci. 1983, 9, 1-76.

62. Crowe, C.T.; Sharma, M.P.; Stock, D.E. Theparticle-source-incell(PSI Cell) model for gas-droplet flows. J. Fluids Eng. 1977, 99, 325-332.

63. Crowe, C.T. Heat transfer in dispersed-phase flow. In Two Phase Momentum, Heat and Mass Transfer in Chemical, Process and Energy Engineering Systems; Afgan, N.H., Tsiklauri, G.V., Eds.; Hemisphere: McGraw-Hill: New York, NY, USA, 1978; Volume 1, pp. 23-32.

64. Shuen, J.S.; Chen, L.D.; Faeth, G.M. Evaluation of a stochastic model of particle dispersion in a turbulent round jet. AIChE J. 1983, 29, 167-170.

65. Shuen, J.S.; Chen, L.D.; Faeth, G.M. Predictions of the structure of turbulent, particle. Laden, round jets. AIAA J. 1983, 21, 1483-1484.

66. Novozhilov, V. On some integrable cases of particle motion in a fluid, mathematics in engineering. Sci. Aerosp. 2010, 1, 371-380.

67. Novozhilov, V. Flashover control under fire suppression conditions. Fire Saf. J. 2001, 36, 641-660.

68. Putnam, A. Integrable form of droplet drag coefficient. Am. Rocket Soc. J. 1961, 31, 1467-1468.

69. Faeth, G.M. Evaporation and Combustion of Sprays. Prog. Energy Combust. Sci. 1983, 9, 1-76.

70. Shirolkar, J.S.; Coimbra, C.F.M.; McQuay, M.Q. Fundamental aspects of modeling turbulent particle dispersion in dilute flows. Progr. Energy Combust. Sci. 1993, 22, 363-399.

71. Ranz, W.E.; Marshall, W.R., Jr. Evaporation from drops: Part I. Chem. Eng. Progress. 1952, 48, 141-146.

72. Ranz, W.E.; Marshall, W.R., Jr. Evaporation from drops: Part II. Chem. Eng. Progress. 1952, 48, 173-180.

73. Faeth, G.M.; Lazar, R.S. Fuel droplet burning rates in a combustion gas environment. AIAA J. 1971, 9, 2165-2171. 
74. Migdal, D.; Agosta, V.D. A Source Flow Model for Continuum Gas-Particle Flow. J. Appl. Mech. 1967, 34, 860-865.

75. Kumar, S.; Heywood, G.M.; Liew, S.K. Superdrop Modelling of a Sprinkler Spray in a Two-phase Cfdparticle Tracking Model. In Proceedings of the Fifth International Symposium on Fire Safety Science, Melbourne, Australia, 3-7 March 1997; pp. 889-900.

76. Lin, C.L.; Chiu, H.H. Numerical Analysis of Spray Combustion in Hybrid Rocket, AIAA 95-2687. In Proceedings of the 31st AIANASMUSAUASEE Joint Propulsion Conference and Exhibition, San Diego, CA, USA, 1995.

77. Kawamura, T.; Kuwahara, K. Computation of High Reynolds Number Flow Around A Circular Cylinder with Surface Roughness. In Proceedings of the 22nd American Institute of Aeronautics and Astronautics, Aerospace Sciences Meeting, Reno, NV, USA, 9-12 January 1984.

78. Boris, J.P.; Grinstein, F.F.; Oran, E.S.; Kolbe, R.L. New insights into large eddy simulation. Fluid Dyn. Res. 1992, 10, 199-228.

79. van Leer, B. Towards the ultimate conservative difference scheme V: A second-order sequel to Godunov's method. J. Computat. Phys. 1979, 32, 101-136.

80. Shu, C.W.; Osher, S. Efficient implementation of essentially non-oscillatory shock-capturing schemes, I. J. Comput. Phys. 1988, 77, 439-471.

81. Shu, C.W.; Osher, S. Efficient implementation of essentially non-oscillatory shock-capturing schemes, II. J. Comput. Phys. 1989, 83, 32-78.

82. Liu, X.D.; Osher, S.; Chan, T. Weighted essentially non-oscillatory schemes. J. Comput. Phys. 1994, 115, 200-212.

83. Jiang, G.S.; Shu, C.W. Efficient implementation of weighted ENO schemes. J. Comput. Phys. 1996, 126, 202-228.

84. Deng, X.G.; Zhang, H.X. Developing high-order weighted compact nonlinear schemes. J. Comput. Phys. 2000, 165, 22-44.

85. Drikakis, D.; Hahn, M.; Mosedale, A.; Thornber, B. Large eddy simulation using high-resolution and high-order methods. Philos. Trans. R. Soc. A 2009, 367, 2985-2997.

86. Hahn, M.; Drikakis, D. Implicit large-eddy simulation of swept-wing flow using high-resolution methods. AIAA J. 2009, 47, 618-630.

87. Panaras, A.G.; Drikakis, D. High-speed unsteady flows around spiked-blunt bodies. J. Fluid Mech. 2009, 632, 69-96.

88. Shimada, Y.; Thornber, B.; Drikakis, D. High-order implicit large eddy simulation of gaseous fuel injection and mixing of a bluff body burner. Comput. Fluids 2011, 44, 229-237.

89. Gordon, S.; McBride, B.J. Computer Program for Calculation of Complex Chemical Equilibrium Compositions and Applications, II. Users Manual and Program Description; Nasa Reference Publication 1311; Lewis Research Center: Cleveland, OH, USA, 1996.

90. Liou, M.S.; Steffen, C.J., Jr. A new flux splitting scheme. J. Comput. Phys. 1993, 107, $23-39$.

91. Liou, M.S. A sequel to AUSM, Part II: AUSM+-up for all speeds. J. Comput. Phys. 2006, 214, 137-170.

92. Shima, E.; Kitamura, K. On New Simple Low-Dissipation Scheme of AUSM-Family for All Speeds; AIAA Paper 2009-136; AIAA: Reston, VA, USA, 2009. 
93. Roe, P.L. Characteristic-based schemes for the Euler equations. Annu. Rev. Fluid Mech. 1986, 18, $337-365$.

94. van Leer, B. Towards the ultimate conservative difference scheme II: Monotonicity and conservation combined in a second-order scheme. J. Comput. Phys. 1974, 14, 361-370.

95. Anderson, W.K.; Thomas, J.L.; van Leer, B. A Comparison of Finite Volume Flux Vector Splittings for the Euler Equations; AIAA Paper 85-122; AIAA: Reston, VA, USA, 1985.

(C) 2011 by the authors; licensee MDPI, Basel, Switzerland. This article is an open access article distributed under the terms and conditions of the Creative Commons Attribution license (http://creativecommons.org/licenses/by/3.0/). 